\title{
An updated roadmap for the integration of metal-organic frameworks with electronic devices and chemical sensors
}

\author{
Ivo Stassen, $\quad{ }^{a b}$ Nicholas Burtch, $\quad{ }^{c}$ Alec Talin, ${ }^{c}$ Paolo Falcaro, de Mark Allendorf ${ }^{c}$ and Rob Ameloot $\quad \star^{a}$
}

\begin{abstract}
Metal-organic frameworks (MOFs) are typically highlighted for their potential application in gas storage, separations and catalysis. In contrast, the unique prospects these porous and crystalline materials offer for application in electronic devices, although actively developed, are often underexposed. This review highlights the research aimed at the implementation of MOFs as an integral part of solid-state microelectronics. Manufacturing these devices will critically depend on the compatibility of MOFs with existing fabrication protocols and predominant standards. Therefore, it is important to focus in parallel on a fundamental understanding of the distinguishing properties of MOFs and eliminating fabrication-related obstacles for integration. The latter implies a shift from the microcrystalline powder synthesis in chemistry labs, towards film deposition and processing in a cleanroom environment. Both the fundamental and applied aspects of this two-pronged approach are discussed. Critical directions for future research are proposed in an updated high-level roadmap to stimulate the next steps towards MOF-based microelectronics within the community.
\end{abstract}

\footnotetext{
${ }^{a}$ Centre for Surface Chemistry and Catalysis, KU Leuven - University of Leuven, Celestijnenlaan 200F, B-3001 Leuven, Belgium. E-mail: rob.ameloot@kuleuven.be

${ }^{b}$ Imec, Kapeldreef 75, B-3001 Leuven, Belgium

${ }^{c}$ Sandia National Laboratories, Livermore, CA 94551-0969, USA

${ }^{d}$ Institute of Physical and Theoretical Chemistry, Graz University of Technology, Stremayrgasse 9, 8010 Graz, Austria

${ }^{e}$ Department of Chemistry, The University of Adelaide, Adelaide, South Australia 5005, Australia
}

\section{Introduction}

The structure and dynamics of matter at the nanometer scale form the basis for both natural processes and technological applications. For instance, ions moving across a lipid membrane and in between electrodes are essential to living cells and batteries, respectively. Similarly, our sense of smell and detectors for hazardous substances are both based on the recognition of

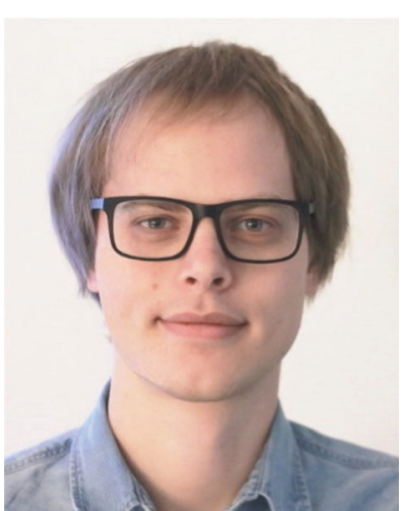

Ivo Stassen
Ivo Stassen is a Postdoctoral Fellow of the Research Foundation - Flanders (FWO) working at the University of Leuven and Imec. He received his $\mathrm{MSc}$ in Bioscience Engineering at KU Leuven in 2012, followed by a PhD in 2016. In 2014-2015, he worked a as visiting scholar at Kyoto University. His research interests include solvent-free and gas-phase synthesis of metalorganic frameworks, host-guest properties and fabrication of functional nanoporous structures such as thin films, patterns and devices.

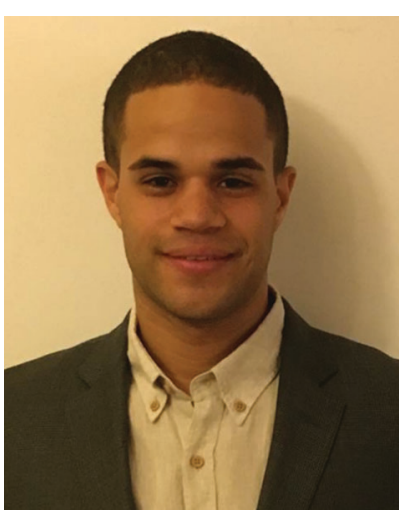

Nicholas Burtch
Nicholas Burtch is a Harry $S$. Truman Fellow at Sandia National Laboratories in Livermore, $\mathrm{CA}$. $\mathrm{He}$ received his BSE from the University of Michigan and $\mathrm{PhD}$ from the Georgia Institute of Technology, both in chemical engineering. His research interests include the experimental synthesis and computational understanding of crystalline, nanoporous materials for materials science and adsorption applications. 
small molecules. To optimize interactions and processes at this length scale, one would ideally be able to structure matter at the (sub-)nanometer level. Indeed, in living organisms, precisely tuned nano-environments are provided by ion channels and dedicated receptors that are formed through self-assembly of molecular building blocks. Man-made devices, on the other hand, typically rely on much cruder fabrication tools: even cutting-edge lithographic techniques fall at least an order of magnitude short in resolution to structure matter at the desired length scale.

Nevertheless, precisely controlled nano-environments do exist in synthetic materials. Metal-organic frameworks (MOFs) are crystalline nanoporous materials built out of metal-based nodes and multitopic organic ligands (also called linkers) connected by coordination bonds (Fig. 1a). ${ }^{1}$ Over the last two decades, MOFs have grown into promising materials for a wide range of potential applications in industry and society. The chemistry of these materials is characterized by the features of their high-surface area crystal lattices: short and long range ordering, intrinsic nanoporosity with pores of tunable size, versatile host-guest chemistries ("guest@MOF" properties) and responsiveness to physical and chemical stimuli. What is more, the synthetic chemistry that enables these features is based on the spontaneous self-assembly of simple molecular building units, which permits the "design" of MOF lattices based on linkers and nodes with known geometries and coordination environments. Hence, MOFs as a high-surface area material platform, at the interface between hard and soft,

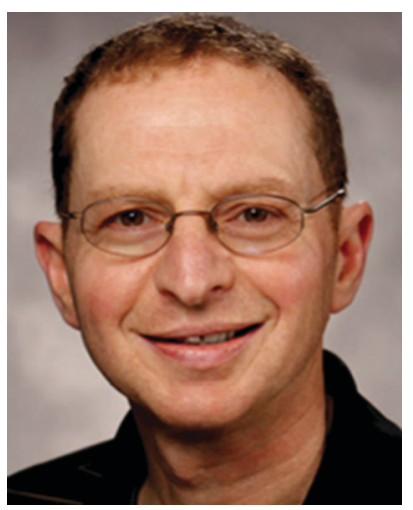

Alec Talin
Alec Talin has been a member of the technical staff at Sandia National Laboratories since 2002. $\mathrm{He}$ is also an adjunct associate professor of materials science and engineering at the University of Maryland. He received a BA degree in chemistry from the University of California, San Diego in 1989, and a PhD degree in materials science and engineering from the University of California, Los Angeles, in 1995. Prior to joining Sandia, he spent six years at Motorola Labs, and was a project leader at the Center for Nanoscale Science and Technology at the National Institute of Standards and Technology. His research focuses on areas of novel electronic materials, energy storage and conversion, and national security. He is a principal editor of the journal MRS Communications.

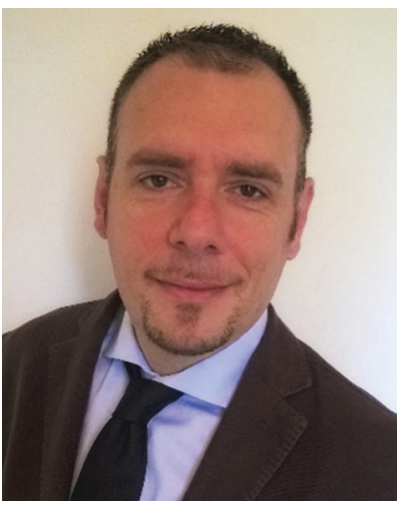

Paolo Falcaro

Paolo Falcaro is professor in Biobased Materials and Technologies at Graz University of Technology (TU Graz - Graz, Austria). He received his $P h D$ in materials engineering in 2006 from Bologna University, Italy. From 2005 to 2009 he worked at Civen/Nanofab (Venice, Italy) as manager of the sol-gel technology for industrial applications. In 2009 he joined CSIRO (Melbourne, Australia), extending the expertise from sol-gel and device fabrication to metal-organic frameworks. In 2011 he received the ARC DECRA, progressing from group leader to team leader in 2014. In 2016 he joined the Institute of Physical and Theoretical Chemistry at TU Graz.

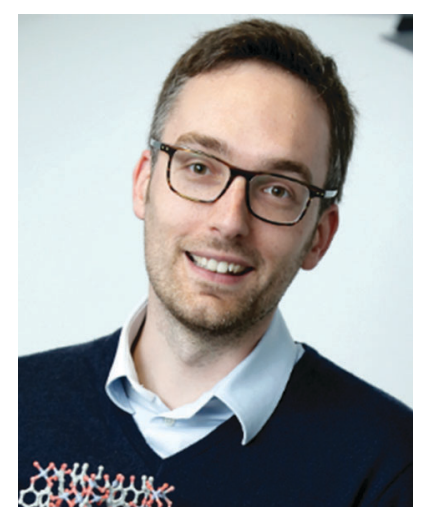

Rob Ameloot
Rob Ameloot obtained his PhD in Bioscience Engineering/Catalytic Technology at KU Leuven (Belgium) in 2011. In 20122013, he worked with Jeffrey Long as a Fulbright postdoctoral fellow at UC Berkeley (US). Currently, he is a tenure-track research professor at the KU Leuven Centre for Surface Chemistry and Catalysis. He was awarded an ERC starting grant to work on bringing metal-organic frameworks from the chemistry lab into 160 publications, including more than 120 journal articles. He is President Emeritus and Fellow of The Electrochemical Society and his awards include a 2014 R\&D 100 Award for a novel approach to radiation detection. the microelectronics fab by developing vapor phase thin film deposition routes. In general, he is passionate about pushing the envelope in porous materials and process technology, with a healthy disregard for traditional subject boundaries. 

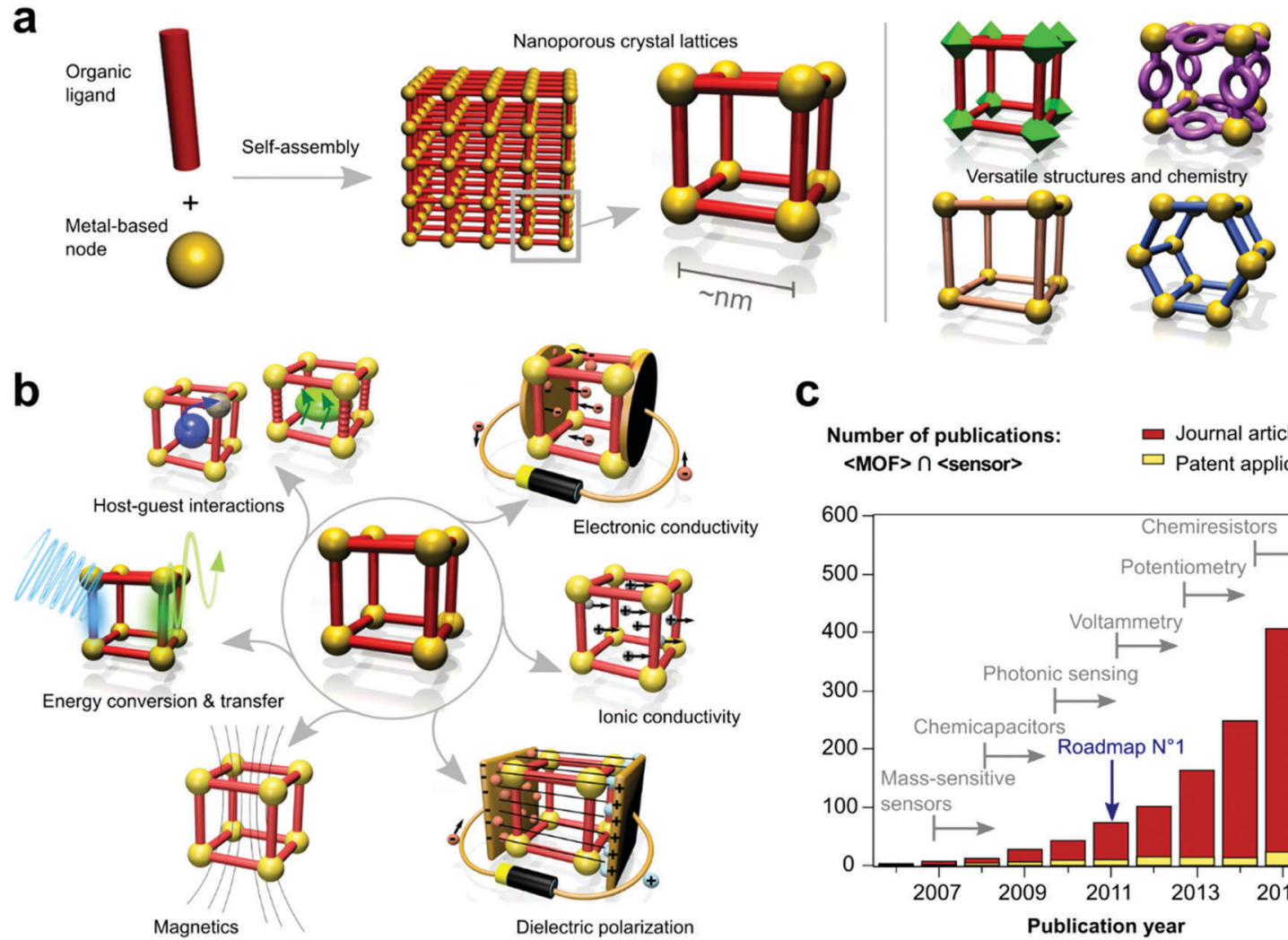

\section{C}
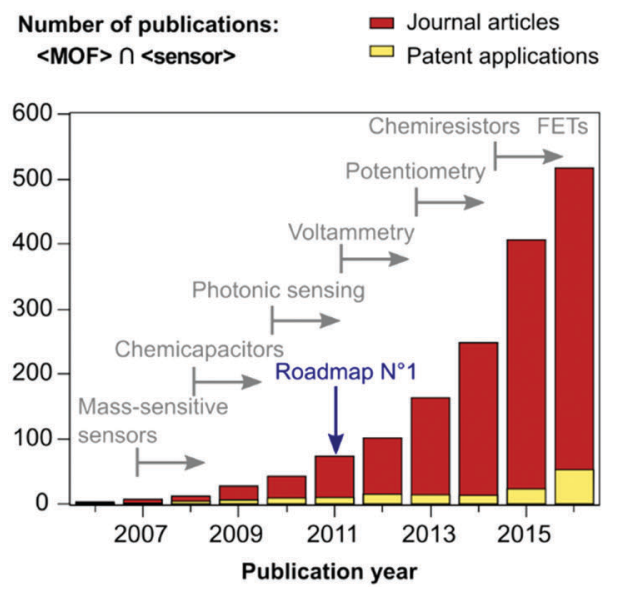

Fig. 1 Graphical representation of MOF, their structural and chemical versatility and some representative concepts related to device applications. (a) MOFs are ordered frameworks built from interconnected organic ligands and metal-based nodes. The broad-scope recommended definition of a MOF: "Coordination network with organic ligands containing potential voids; coordination network being a coordination compounds extending in at least one dimension through repeating coordination entities". ${ }^{6}$ (b) Some key properties of MOFs that may lead to applications in electronic devices. (c) The burgeoning field of MOF devices visualized through the seminal example of chemical sensors. Shown is the evolution of the yearly publications that combine the concepts "metal-organic framework" and "sensor" (source: SciFinder ${ }^{\mathbb{R}}$ ). The earliest demonstrations of some general concepts, as well as the 2011 roadmap article (no. 1), are positioned on the same timeline.

inorganic and organic materials, offer a new window for finetuning various structure-property relationships.

MOFs are typically obtained as microcrystalline powders through solvothermal synthesis. Therefore, MOF research initially focused on evaluating and optimizing the physical and chemical properties in the context of bulk applications: gas storage and chemical engineering operations (catalysis, separation). ${ }^{2,3}$ These fields build on the prior research and industrial implementation of related materials, e.g. nanoporous silicates such as zeolites. More recently, the unique properties of MOFs encouraged research lines atypical for porous materials, often in areas where the introduction of ordered porosity promises new concepts entirely. In contrast to the proposed bulk applications of MOFs, these more recent directions frequently target highvalue technological areas requiring very little material in comparison. Examples include MOFs as functional materials in chemical analytics, biomedical technology, solid-state material physics and various other branches of nanoscience and technology. ${ }^{4}$

This review highlights the research aimed at the implementation of MOFs as an integral part of solid-state devices. In this context, "integration" denotes that the MOF is an integral component of the actual device structure and that the fundamental aspects of device or circuit design are being taken into account at least rudimentarily. We focus in particular on electronic devices, which broadly defined are physical entities that influence electrons when connected to an electrical circuit. Examples of electronic devices include individual components such as resistors, transistors and diodes, as well as assemblies of such components such as amplifiers, sensors and microcontrollers. Our everyday life increasingly relies on ever more capable electronic devices including smartphones and computer processors based on millions of miniaturized logic units, memory arrays and input/output interfaces such as physical sensors, actuators and displays. The enormous success of electronic devices has mainly been enabled by low-cost production through scalable "CMOS" microfabrication (CMOS = complementary metal-oxide-semiconductor). The renowned "Moore's law" stating that the number of transistors in integrated circuits doubles every two years, has been a self-imposed driving force for CMOS research and downsizing in academia and industry since the 1970s. Importantly, this downsizing course has not only been enabled by improved fabrication tools, but to an equal extent by advances in materials science that ensure reliable performance of ever tinier circuit elements. Industrial and academic research efforts in this context have for long been harmonized by the 
International Technology Roadmap for Semiconductors (ITRS). These reports composed by semiconductor industry experts specify research directions and targets in order to meet needed material, fabrication and device specifications. In 2011, these guidelines inspired some of us to stipulate a first roadmap for exploratory research on MOFs in electronics. ${ }^{5}$ Today, circa five years of research later, this review provides an updated overview. Analogous to the previous paper, progress in different areas of interest is reviewed and perspectives and suggested focus points are stipulated. Although arguably arbitrary, we strictly limit this discussion to MOFs and exclude most of the larger family of coordination polymers, most of which are nonporous. ${ }^{6}$

One of the key trends in microelectronics is a gradual shift from inorganic and silicon-centered, towards organic and hybrid organic-inorganic devices. Depending on the application scenario, this route is followed either to benefit from the greater compositional complexity of organics, to reduce production costs, or to push downsizing to the limit, down to molecular dimensions. In the context of digital logic, such approaches are referred to as "beyond CMOS", as CMOS-based scaling in silicon technology will run into fundamental limitations. Technologies based on new materials such as graphene promise scaling beyond the limitations of silicon and are on the verge of causing paradigm shifts in several areas. ${ }^{7}$ Application areas in which similar transitions are either impending or already commercially implemented include photovoltaic cells ( $c f$. from silicon to perovskite or dye-sensitized solar cells) and displays ( $c f$. from indium tin oxide to polymers). This shift in materials scope is accompanied by a rising interest in molecular approaches and a rising acceptance and economic viability of alternative manufacturing approaches such as inkjet printing and roll-to-roll manufacturing. In our opinion, the continuous exploration of new materials and properties combined with an increasing attention for bottom-up strategies at a length scale where chemistry and materials science merge could make electronic devices one of the most interesting proving grounds for MOFs.

Directly related to their structure, MOFs offer properties outside the existing materials scope in electronics: e.g. sensor coatings with adsorption properties geared to specific analytes, dielectric properties tunable at will, intrinsic porosity that allows through-solid mass and ion transport, mechanical properties in between polymers and inorganics and electronic conductance tunable from practically zero to over a hundred $\mathrm{S} \mathrm{cm}^{-2}$ (Fig. 1b). Within this range of potential applications, chemical sensing seems to be the first to shift from proof of concept to the development stage, arguably due to the directly transferable adsorption studies available from researching bulk applications. Nevertheless, recent milestones such as the design and synthesis of a first generation of highly conductive MOFs (2014-2015) and the fabrication of a field effect transistor, FET, (2016) highlight the potential of MOFs in future electronic devices (Fig. 1c). The primary prerequisite for realizing such MOF-based devices is a radical performance increase over established technologies. Moreover, manufacturing these devices will critically depend on the compatibility of MOFs with existing fabrication protocols and predominant standards. Therefore, it is important that research efforts focus in parallel on a fundamental understanding of the distinguishing properties of MOFs and eliminating fabrication-related obstacles for integration. For MOFs, the latter implies a shift from the microcrystalline powder synthesis in chemistry labs, towards film deposition and processing in a cleanroom environment. Moreover, it is important that the field moves beyond the synthetic and structural aspects to engage experts in other fields, such as materials science, physics, and electrical engineering and to realize the use of MOFs in devices.

In this review, both aspects of the two-pronged approach suggested above are highlighted. Progress in understanding relevant fundamental physical properties of MOFs is discussed first. Secondly, the general context of device microfabrication is outlined and relevant MOF research is put in perspective. Thirdly, case studies of MOF integration in electronic devices are discussed for different application fields. Lastly, general progress of the field, outstanding obstacles and perspectives are outlined.

\section{Fundamental MOF properties}

A crucial requirement for designing and fabricating electronic devices incorporating MOFs is knowledge of their basic charge transport, photonic, and magnetic properties. Compared to established materials such as silicon and organic semiconductors, little is known about MOFs. However, considerable progress has been made since the original 2011 roadmap, ${ }^{5}$ providing ample reason to envision that MOFs could have a prominent place in future electronic devices. Several review articles already provide in-depth summaries of various aspects of MOF research that are relevant to electronic applications. Our intention here is not to exhaustively cover the same ground, but rather to discuss recent milestones that highlight progress and to identify opportunities and critical needs for advancing the field. Relevant review articles for further reading will be cited where applicable. Following the structure of the first-generation roadmap, in this section we update the status of charge transport, optical, and light generation. New sections concerning magnetic properties and light harvesting are also included.

\subsection{Electronic conductivity}

The ability to conduct electrical charge is perhaps the most important and also least explored property of MOFs to developing them as active materials in electronic devices. Most MOFs are electrical insulators, but in the last five years this subcategory has expanded from merely two structures in 2011 to about two dozen materials with demonstrated conductivity or charge mobility. This ten-fold increase motivated a recent review article by Dincă and coworkers that identifies strategies for designing electronically conducting MOFs. ${ }^{8}$ These results, although limited, are nevertheless highly encouraging. However, the best MOF conductivities obtained so far are still low by comparison with other types of conducting materials (Table 1). 
Table 1 Comparison of conductivity data for a selection of MOFs and other conducting materials

\begin{tabular}{|c|c|c|c|c|}
\hline Material or formula unit & Conductivity $\left(\mathrm{S} \mathrm{cm}^{-1}\right)$ & Charge carrier ${ }^{e}$ & Mobility $\left(\mathrm{cm}^{2} \mathrm{~V}^{-1} \mathrm{~s}^{-1}\right)$ & Ref. \\
\hline Copper & $10^{5}-10^{6}$ & $\mathrm{e}$ & 46 & 41 \\
\hline Doped polyacetylene & $560(\mathrm{n}) ; 360(\mathrm{p})$ & $\mathrm{h}$ or $\mathrm{e}$ & 1 (n-doped, cis) & 42 \\
\hline Undoped polyacetylene & $10^{-9}$ & & & 42 \\
\hline Doped polyaniline & $10^{3}$ & & & 41 \\
\hline Graphene & $550^{b}$ & & & 41 \\
\hline Polycrystalline graphite & 1250 & & & 43 \\
\hline Polythiophenes & 1975 & $\mathrm{~h}$ & $1-10$ & 29 and 44 \\
\hline Rubrene & & & 4 & 45 \\
\hline TTF-TCNQ & 700 & & & 41 \\
\hline $\mathrm{Cu}\left[\mathrm{Ni}(\mathrm{PDT})_{2}\right]$ ( $\mathrm{I}_{2}$ doped $)$ & $1 \times 10^{-4 a}$ & & & 12 \\
\hline $\mathrm{Cu}_{3}(\mathrm{BHT})_{2}$ & $1580^{b}$ & h or $\mathrm{e}$ & $99(\mathrm{~h}) ; 116(\mathrm{e})$ & 46 \\
\hline $\mathrm{Ni}_{3}(\mathrm{HITP})_{2}$ & $2 ;^{a} 40^{b}$ & $\mathrm{~h}$ or $\mathrm{e}$ & 48.6 & 28 and 31 \\
\hline $\mathrm{Ni}_{3}(\mathrm{BHT})_{2}$ & $0.15 ;^{a} 2.8-160^{c}$ & & & 47 and 48 \\
\hline $\mathrm{Mn}_{2}(\mathrm{DOBDC})$ & $3.9 \times 10^{-13 a}$ & & & 49 \\
\hline $\mathrm{Fe}_{2}(\mathrm{DOBDC})$ & $3.2 \times 10^{-7 a}$ & & & 49 \\
\hline $\mathrm{Mn}_{2}(\mathrm{DSBDC})$ & $1.2 \times 10^{-12 a}$ & & 0.01 & 49 and 50 \\
\hline$\left\{\left[\mathrm{Cd}_{2}(\mathrm{AZBPY})_{2}(\mathrm{HO}-1,3-\mathrm{BDC})_{2}\right](\mathrm{AZBPY})\left(\mathrm{H}_{2} \mathrm{O}\right)\right\}_{n}$ & $1.86^{b}$ & $\mathrm{e}$ & & 51 \\
\hline $\mathrm{K}_{1.2} \mathrm{Ru}_{3.6}\left[\mathrm{Ru}(\mathrm{CN})_{6}\right]_{3} \cdot 16 \mathrm{H}_{2} \mathrm{O}$ & $5.7 \times 10^{-3}$ & & & 36 \\
\hline TCNQ@Cu $3(\mathrm{BTC})_{2}$ & $0.07^{b}$ & $\mathrm{~h}$ & & 18 \\
\hline TCNQ@[Cu(TPyP $\left.) \mathrm{Cu}_{2}\left(\mathrm{O}_{2} \mathrm{CCH}_{3}\right)_{4}\right]$ & $2.5 \times 10^{-6}$ & & & 34 \\
\hline $\mathrm{Zn}_{2}$ (TTFTB) & $4.0 \times 10^{-6}$ & & 0.2 & 22 \\
\hline Porphyrin Zn-SURMOF-2 & & $\mathrm{h}$ & 0.002 & 52 \\
\hline Pd@porphyrin Zn-SURMOF-2 & & $\mathrm{h}$ & $0.003-0.004$ & 52 \\
\hline$\left[\mathrm{Sr}(\mathrm{HBTC})\left(\mathrm{H}_{2} \mathrm{O}\right)\right]_{n}$ & $10^{-9}-10^{-7 a}$ & & & 32 \\
\hline NNU-7 (anthracene MOF) & $1.3 \times 10^{-3 c}$ & & & 24 \\
\hline$\left(\mathrm{NBu}_{4}\right)_{2} \mathrm{Fe}_{2}(\mathrm{DHBQ})_{3}$ & $0.16^{a}$ & & & 15 \\
\hline
\end{tabular}

Sample morphologies used for conductivity measurements, if specified: ${ }^{a}$ pellet; ${ }^{b}$ film; ${ }^{c}$ microflakes or single crystal. ${ }^{d}$ Coordination polymer without any indication of guest-accessible porosity. ${ }^{e}$ h: hole; e: electron.

Although nonporous coordination polymers such as poly(Ni 1,1,2,2-ethenetetrathiolate), a rare example of a hybrid n-type semiconductor, are certainly of interest, we confine the discussion here to conducting MOFs that are clearly nanoporous. The next two sections provide first a brief introduction to charge transport from a solid-state physics point of view and its connection with MOFs, then a summary of charge transport mechanisms known to be operative in MOFs.

Fundamentals of charge transport. According to band theory, solids can be classified as insulators, semiconductors, or metals based on the magnitude of the energy gap, $E_{\mathrm{g}}$, separating the valence band (VB) from the conduction band (CB): $E_{\mathrm{g}}>4 \mathrm{eV}$ are insulators, $0<E_{\mathrm{g}}<3 \mathrm{eV}$ are semiconductors, and $E_{\mathrm{g}}<0$ (i.e. partially filled band) are metals. ${ }^{9}$ The band model works well for many solids in which strong electronic coupling between neighboring atoms leads to large band dispersion (i.e. large $\Delta E$ between bonding and antibonding orbitals) that makes it energetically favorable to delocalize carriers across many lattice sites. For solids with large band dispersion (also known as "bandwidth") such as Si (Fig. 2a), the energy gained by delocalizing the charge $(\sim W / 2)$ makes the electron-electron repulsion effects insignificant. In metals, the repulsion effects relative to Si are further reduced electrostatically by the large density of free carriers. However, true metallic conduction (i.e. decreasing conductivity with increasing temperature) is not observed in many materials with bandwidth less than $\sim 0.5 \mathrm{eV}$. This is the case even when the Fermi level moves into the VB or the CB due to strong carrier localization; here, the carriers are energetically
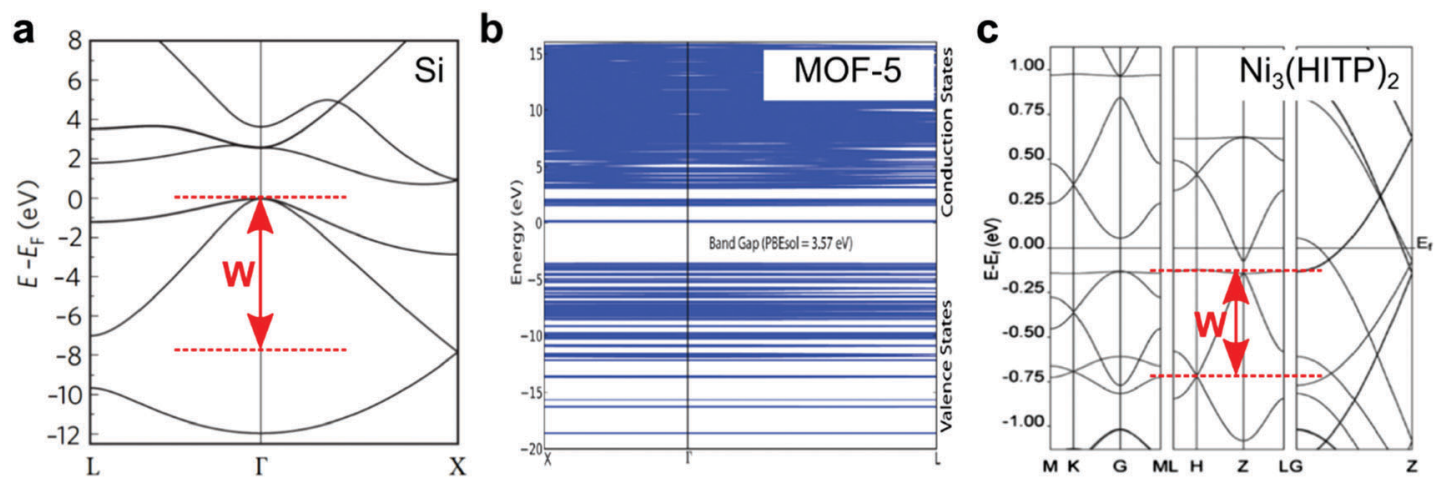

Fig. 2 Calculated band diagrams. (a) Si. Adapted from ref. 9. Copyright 2011 Springer. (b) MOF-5. Reproduced with permission from ref. 10. (c) $\mathrm{Ni}_{3}(\mathrm{HITP})_{2}$. Reproduced with permission from ref. 14. Copyright 2016 American Chemical Society. The bandwidth $(W)$ is approximately 8 eV, 0 eV, and $0.8 \mathrm{eV}$, respectively. 
trapped on lattice sites and require thermal agitation to hop from site to site.

Typical MOFs, with low atomic density and the strong localization of the electron wave function characteristic of Werner-type coordination complexes, by contrast have little or no band dispersion. They thus behave as insulators, as is the case of MOF-5 (Fig. 2b). ${ }^{10}$ This is true for many solids in which there is far less electronic coupling than in Si due to large differences in orbital energy between the bonding atoms and/or reduced orbital overlap typical of d-electron compounds. In these cases, the conductivity classifications based solely on band gap can be misleading. For example, $\mathrm{MnO}$ and $\mathrm{CrO}$ have partially filled d-bands with an odd number of d-electrons, implying that, irrespective of the ligand field splitting, they should be metallic. Yet these and many other transition metal oxides are insulators at room temperature due to the strong electrostatic repulsion that charge carriers experience when forced to doubly occupy a d-orbital in the process of moving across the lattice. ${ }^{11}$

In materials like MOFs with limited electronic coupling and narrow bandwidth, electron-electron repulsion becomes important, and in many transition metal compounds this causes the CB to split into two sub-bands separated by the Hubbard energy, referred to as the lower and the upper Hubbard bands. Hubbard insulators can be converted to conductors by formation of anion or cation vacancies or by impurity doping; this strategy has been implemented in some MOFs. ${ }^{12}$ Prussian blue (PB), composed of Fe(II) and Fe(III) ions coordinated by cyanide ligands, is an excellent example of this. $\mathrm{PB}$ is expected to be a band insulator due to the large ligand field splitting, but one would also predict that it should become metallic when oxidized to Berlin green or reduced to Prussian white. Nevertheless, both oxidized and reduced PB exhibit thermally activated (hopping) conduction due to formation of small polarons. ${ }^{13}$ Small polarons form when the electrostatic energy gained in distorting the lattice by the charge carrier exceeds the energy gained by carrier delocalization (i.e. the bandwidth) leading to carrier trapping at individual lattice sites. Typical polaron mobilities are $\ll 1 \mathrm{~cm}^{2} \mathrm{~V}^{-1} \mathrm{~s}^{-1}$, compared with $>100 \mathrm{~cm}^{2} \mathrm{~V}^{-1} \mathrm{~s}^{-1}$ measured for elemental and compound semiconductors. Oxidation and reduction in PB are effectively a form of oxidative or reductive doping; oxidation decreases EF, leading to a higher concentration of hole carriers, whereas reduction increases $\mathrm{EF}$, producing a higher concentration of electrons.

In summary, understanding conductivity in the context of materials with long-range order provides an alternative perspective on MOF design from the traditional chemist's viewpoint of localized molecular orbitals. Charge localization and low band dispersion in MOFs therefore motivates two basic strategies for designing conducting frameworks. One can attempt to increase band dispersion by increasing the electronic coupling between the metal ions and the linkers. Alternatively, a doping strategy can be adopted, in which the Fermi level is moved to increase the carrier concentration in the band gap, either by doping or introducing defects (anion or cation vacancies).

Charge transport mechanisms in MOFs. In brief, three charge transport mechanisms have been identified in MOFs (Fig. 3a): through-bond conduction, through-space conduction (charge delocalization due to the close approach of adjacent aromatic linkers), and guest molecules. In through-bond conduction, charge moves through continuous chains of covalent and coordination bonds in the material. Examples include frameworks with redox-active components (linkers, metal ions, or both), ${ }^{12,15}$ and two dimensional graphene-like frameworks that exhibit extended $\pi$-conjugation. ${ }^{31}$ Typically, this involves so-called "hopping transport" and is characterized by a strong temperature dependence. ${ }^{16}$

Tunneling is a form of through-bond conduction, but one in which the barrier between donating and accepting portions of the framework is too high to allow hopping charge transport to occur.
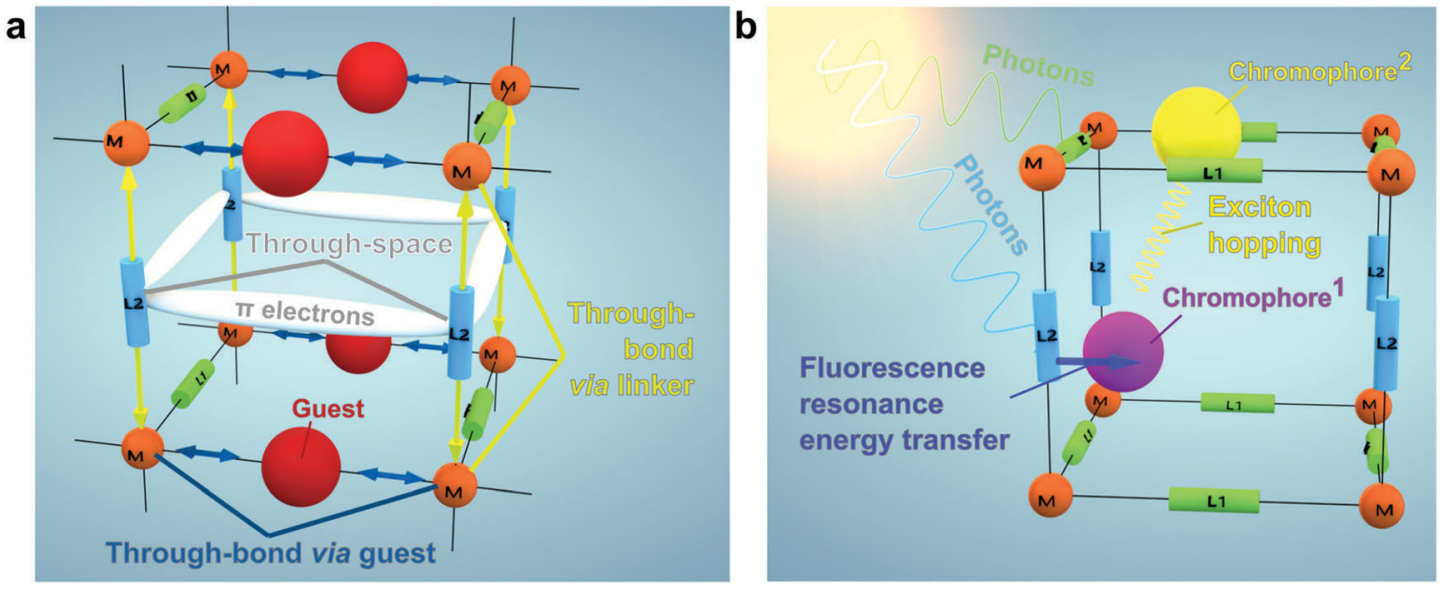

Fig. 3 Schematic illustrations of analogous routes for charge and energy transport in MOFs. (a) Three possible mechanisms have been observed in MOFs for charge transport: through-bond conduction via metal nodes and linkers; through-bond conduction via guests that function as bridging units; and through-space conduction via charge delocalization through adjacent aromatic linkers. (b) Photoexcitation followed by fluorescence resonance energy transfer or exciton hopping between chromophoric framework units or guest. 
It is characterized by a weak temperature dependence and is operative over short distances (1-4 nm). ${ }^{16}$ We are unaware of any MOFs for which tunneling is known to be operative, although a MOF infiltrated with metal nanoclusters is reported to be conductive by tunneling between the nanoclusters. ${ }^{17}$

The use of guest molecules to create new charge transport pathways is a recently discovered charge transport mechanism in MOFs and is related to through-bond conductivity if the guest molecules are strongly bound to the framework. The prototypical example of this is TCNQ (7,7,8,8-tetracyanoquinodimethane) infiltrated HKUST-1, for which spectroscopic and other evidence is consistent with the guest molecules bridging copper dimers by binding to open metal sites. ${ }^{18}$ In such cases (discussed in more detail below) the guest molecule serves as the bridge in a donorbridge-acceptor (DBA; also known as "superexchange") model. ${ }^{19-21}$ A strong temperature dependence of the conductivity would be expected; however, in TCNQ@HKUST-1, the activation energy is only $14 \mathrm{meV}$, suggesting strong coupling among the components of the DBA complex.

Through-space conductivity is known in a few cases: an isoreticular series of MOFs with composition $\mathrm{M}_{2}$ (TTFTB) $(\mathrm{M}=\mathrm{Mn}$, Co, $\mathrm{Zn}$, and $\mathrm{Cd} ; \mathrm{H}_{4}$ TTFTB $=$ tetrathiafulvalene tetrabenzoate $)^{22,23}$ and an anthracene-based MOF that is electroluminescent. ${ }^{24}$ In the $\mathbf{M}_{2}$ (TTFTB) structures, the interplanar linker separation is only $\sim 3.7 \AA$, enabling interlinker charge transfer to occur. Band structure calculations of the zinc version predict that the width of the valence band edge is $400 \mathrm{meV}$; this is considerably greater than that of most MOFs, for which the bands are so narrow that charge can be considered completely localized.

Increasing conductivity with temperature generally suggests hopping conduction; however, band conduction in an intrinsic semiconducting MOF with a band gap of $\sim 0.3 \mathrm{eV}$ or lower could also display this behavior around room temperature. The process of charge hopping between neighboring sites can be described using a configuration coordinate diagram with the $x$-axis representing the distortion coordinate around the site with the trapped carrier. $^{20,25}$ In contrast, decreasing $\sigma$ with temperature indicates band conduction, which could be metallic (for partially filled bands) or semiconducting due to dopants with low $(\ll k T)$ ionization energy. The temperature dependence of $\sigma$ has been measured for only a few MOFs and in all cases increases with temperature. The fairly wide range of activation energies, from as low as $0.1 \mathrm{meV}$ to as high as $810 \mathrm{meV}$, suggests a large variation in the extent of charge delocalization and carrier trapping mechanisms. ${ }^{8}$ Activation energies in the range of several hundred $\mathrm{meV}$ are consistent with formation of small polarons.

Measurements of charge transport properties. Electronic conductivity is described by eqn (1), in which $\sigma$ is the conductivity (in $\mathrm{Ohm}^{-1} \mathrm{~cm}^{-1}=\mathrm{S} \mathrm{cm}^{-1}$ ), $\mu$ is the charge mobility (in $\mathrm{cm}^{2} \mathrm{~V}^{-1} \mathrm{~s}^{-1}$ ), and $N$ is the charge carrier concentration (in $\mathrm{C} \mathrm{cm}^{-3}$ ):

$$
\sigma=\mu N
$$

There are thus four measurable quantities, including the identity of the majority charge carrier (holes or electrons). Of these, $\sigma$ is the most straightforward to measure and several techniques are available. ${ }^{8}$ Obtaining the temperature dependence of $\sigma$ yields insight into the conduction mechanism. Clouding the picture, however, is that the morphology of the sample and the measurement technique used can strongly affect the conductivity value that is experimentally determined. For example, van der Pauw measurements on $\mathrm{Ni}_{3}(\mathrm{BHT})_{2}$ microflakes have yielded a conductivity more than 1000 times higher than two-point probe measurements on pellets. These issues are reviewed elsewhere, ${ }^{8,26}$ but it suffices to say that control of morphology is essential and that versatile synthetic approaches are needed to fully understand intrinsic $v s$. defect- or grain boundary-controlled conductivity of MOFs.

The identity of the charge carrier has only been determined for a few MOFs, most of which are hole conductors. ${ }^{12,27,28}$ Carrier identity can be established directly by Seebeck coefficient or Hall measurements or can be inferred from doping studies. $^{12,22,27}$ Alternatively, a FET device can be used, which also provides the mobility. ${ }^{28}$ Charge mobility has only been measured for a few MOFs. The values reflect the localized nature of charge; two of the reported values are a factor of 10 or more lower than typical organic conducting polymers. ${ }^{29}$ However, the high mobility of $\mathrm{Ni}_{3}(\mathrm{HITP})_{2}$ places it within the range of metals such as copper and higher than many organic conductors (Table 1). Carrier concentrations are virtually unknown; to our knowledge, this property has never been measured for a MOF.

Semiconducting MOFs. Although semiconductivity can be broadly identified by the band gap of a material, as discussed above, here we define semiconductivity as a material having both a band gap and conductivity that increases with temperature. This temperature dependence may not be exponential.

The first convincing demonstration of semiconductivity in a porous MOF was the synthesis of $\mathrm{Cu}\left[\mathrm{Ni}(\mathrm{PDT})_{2}\right]$, a material in which the redox-active metals provide charge mobility through the structure. ${ }^{12}$ This material has the same crystal structure as the previously reported coordination polymer $\mathrm{Cu}\left[\mathrm{Cu}(\mathrm{PDT})_{2}\right] ;^{30}$ however, the pores of that material collapse upon removal of solvent. The optical band gap for the material is about $2.0 \mathrm{eV}$, similar to some organic semiconductors. The conductivity displays a temperature dependence with a relatively large activation energy of $0.49 \mathrm{eV}$, suggesting a weak hopping mechanism in a material with relatively little band dispersion. In this regard, $\mathrm{Cu}\left[\mathrm{Ni}(\mathrm{PDT})_{2}\right]$ is similar to the Prussian Blue example discussed above. The activated MOF is essentially an insulator (conductivity of only $10^{-8} \mathrm{~S} \mathrm{~cm}^{-1}$ ), but this is strongly enhanced upon oxidative doping with iodine (Table 1), indicating that the majority charge carriers are holes (i.e., a p-type conductor).

An exemplary semiconducting MOF in which both throughbond and through-space mechanisms are operative is $\mathrm{Ni}_{3}(\mathrm{HITP})_{2}$, a "metal-organic graphene analogue" (MOG; Fig. 4). ${ }^{31}$ A very narrow band gap $(0.1 \mathrm{eV})$ is found and the conductivity, among the highest known (40 S cm ${ }^{-1}$ for a thin film), is more than nine orders of magnitude higher than the first report of a conducting porous $\mathrm{MOF}, \mathrm{Cu}\left[\mathrm{Ni}(\mathrm{PDT})_{2}\right]$ (Table 1 ). $\mathrm{Ni}_{3}(\mathrm{HITP})_{2}$ consists of $\pi$-stacked two dimensional sheets of 2,3,6,7,10,11-hexaaminotriphenylene linkers coordinated to Ni(II) ions. This and other MOGs assembled from multitopic dithiolene and $o$-semiquinone aromatic organic moieties bridged by square-planar metal ions have a high degree of in-plane charge delocalization, similar to that 


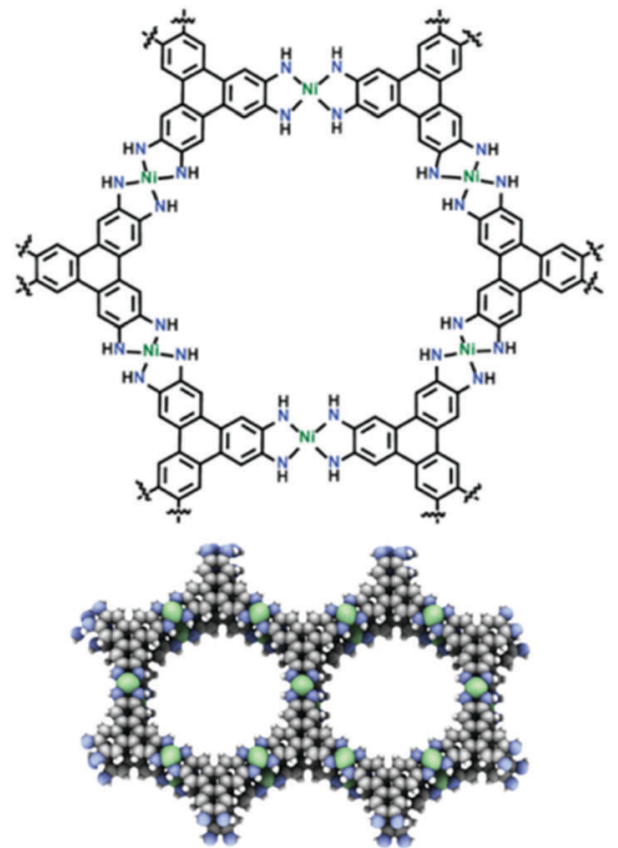

Fig. 4 Structure of the secondary building unit and space-filling image of the metal-organic graphene analogue $\mathrm{Ni}_{3}(\mathrm{HITP})_{2}$.

observed in graphene and layered metal chalcogenites. ${ }^{14}$ In the first report by Dincă and co-workers, temperature-dependent conductivity was observed, with an activation energy of $\sim 0.025 \mathrm{eV},{ }^{31}$ consistent with the relatively narrow bandwidth of $\sim 0.5 \mathrm{eV}$ estimated from the dispersion relation calculated for $\mathrm{Ni}_{3}(\mathrm{HITP})_{2}$ (Fig. 2c). This weak temperature dependence suggests either a tunneling mechanism for charge transport, or strong superexchange-type coupling in which there is extensive mixing between the metal ions and coordinated linkers.

The electrical properties of $\mathrm{Ni}_{3}(\mathrm{HITP})_{2}$ were recently probed using a FET in which the MOF served as the channel. A p-type semiconducting behavior was observed (see Section 4.1). ${ }^{28}$ Interestingly, however, the $I-V$ transfer curve of the FET suggests that the material is ambipolar. A rather high mobility was obtained ( $c a .40 \mathrm{~cm}^{2} \mathrm{~V}^{-1} \mathrm{~s}^{-1}$ ), comparable to or exceeding the best single crystal organic semiconductors at room temperature. This is particularly surprising, given the polycrystalline morphology of the MOF in the channel and the relatively low defect density at the channel-oxide interface $\left(8 \times 10^{12} \mathrm{~cm}^{-2}\right.$, still $\sim 100 \times$ higher than high-quality $\mathrm{Si} / \mathrm{SiO}_{2}$ interfaces in metal oxide semiconductor FETs), suggesting that the intrinsic carrier mobility could be even higher. However, the narrow band gap limits the utility of $\mathrm{Ni}_{3}(\mathrm{HITP})_{2}$ for semiconductor applications.

A Sr-based MOF was also recently reported, consisting of 2-D sheets of $\operatorname{Sr}$ (II) ions bridged by monodentate benzene tricarboxylate linkers in a three dimensional structure. ${ }^{32}$ A bandgap of $2.3 \mathrm{eV}$ was measured, in good agreement with a DFT-predicted indirect gap of $2.04 \mathrm{eV}$. Temperature-dependent conductivity measurements yielded an activation energy of $170 \mathrm{mV}$, indicating hopping transport and consistent with the small dispersion in the VB maximum and CB minimum bands predicted by DFT.
Together, these yield a conductivity of only $10^{-9}-10^{-7} \mathrm{~S} \mathrm{~cm}^{-1}$. Coordinated water molecules evidently played no role in the conductivity, as their removal by heating to $335 \mathrm{~K}$ did not affect the conductivity. Thus, in spite of the large and heavy $\operatorname{Sr}$ (II) cations, charge transport through the carboxylate linkages is still too small to produce significant conductivity.

Emergent electronic properties via guest-molecule infiltration. Recently, a new MOF concept was advanced, "guest@MOF," in which non-innocent guest molecules are used as a design element to install novel properties. ${ }^{21}$ Talin et al. showed that over seven orders of magnitude increase in electronic conductivity occurs when the pores of HKUST-1 are infiltrated with the redox-active molecule TCNQ. ${ }^{18}$ The majority charge carriers were identified from measurement of the Seebeck coefficient to be holes, ${ }^{27}$ qualitatively consistent with a superexchange mechanism enabled by TCNQ molecules bridging copper paddlewheel building units. ${ }^{21}$ Neumann et al., in an independent study, also considered a super exchange mechanism, but speculated that the majority carriers are electrons. ${ }^{33}$ This concept has been extended to other paddlewheel MOFs. A TCNQ-doped copper paddlewheel MOF with porphyrin linkers also shows increased electronic conductivity, although much smaller than seen in TCNQ@HKUST-1. ${ }^{34}$ Increased conductivity is also observed when HKUST-1 is infiltrated with ferrocene; here, the magnitude of the increase is much smaller than with TCNQ and is attributed to improved electronic coupling between the paddlewheel secondary building units. ${ }^{35}$

The family of hexacyanoferrates, including $\mathrm{PB}$, provides additional examples of guest@MOF behavior. Although PB itself is not typically considered a MOF because it lacks porosity upon removal of guest water molecules, Behera et al. synthesized ruthenium-substituted analogues that have permanent porosity and reasonably high surface areas. ${ }^{36}$ These exhibit thermally activated hopping conduction (Table 1), with the $\mathrm{Ru}(\mathrm{III}) \mathrm{Ru}(\mathrm{II})$ having a conductivity $\sim 100 \times$ that of $\mathrm{PB}$ at room temperature. Prior work on the all-iron versions show that control of oxidation state and water content allows conductivity in this system to vary from semiconducting to insulating. ${ }^{13}$ Other examples of emergent properties resulting from a guest@MOF structure have been reported. These include guest-tunable spin crossover, ${ }^{37}$ reversible ferromagnetic-antiferromagnetic behavior, ${ }^{38}$ and resistance switching. ${ }^{39}$ Reviews of these and other guest-induced phenomena are presented elsewhere. ${ }^{21,40}$

Theoretical predictions of charge transport in MOFs. Firstprinciples calculations of MOF electronic structure, primarily using DFT, time-dependent DFT, and band-structure theory, are providing new insight into the design of conducting MOFs. In some ways, theory and experiment are catching up to work done decades ago to understand the electronic structure of transition metal complexes. For example, $\mathrm{Gu}$ et al. combined DFT calculations with ellipsometry measurements to probe the electronic structure of HKUST-1. ${ }^{53}$ The calculated values of the HOMO-LUMO gap in the isolated BTC (= benzene-1,3,5tricarboxylate) linker compared with the predicted excitation spectrum for the MOF indicate that the absorption bands observed by ellipsometry are the result of transitions from the 
linker to the empty d orbitals on the $\mathrm{Cu}$ (II) ions (ligand-to-metal charge transfer; LMCT). This is not a surprise; the building unit in this MOF has a molecular analogue in copper acetate, for which the charge transfer and ligand field transitions have been characterized in great detail. ${ }^{54,55}$ Nevertheless, as will be discussed below, the number of MOFs for which the band structure has been predicted has grown considerably. New computational approaches specific to MOFs are also being developed. For example, Walsh and coworkers developed a method to compute the electrochemical potentials of MOFs, which enables the alignment of the valence and conduction bands with other electronic materials, such as $\mathrm{TiO}_{2}$, to be assessed. ${ }^{56}$

DFT calculations demonstrate that bandgap modulation is possible. The band gaps of IRMOFs $\left(\mathrm{Zn}_{4} \mathrm{O}\right.$ node), ${ }^{57-61}$ UiO$66,{ }^{62,63} \mathrm{MFU}-4,{ }^{64} \mathrm{Zn}$-DCPP SURMOF- $2,{ }^{65} \mathrm{Ni}_{3}(\mathrm{HITP}){ }_{2},{ }^{14}$ and novel hybrid perovskite MOFs ${ }^{66}$ can be tuned by modifying the linker or metal ion, applying strain, or by changing the counter ions in the pores. With the exception of $\mathrm{Ni}_{3}(\mathrm{HITP})_{2}$, there is very little dispersion in the band structure predicted for these materials, indicating that charge transport, if it occurs, would most likely be by a hopping mechanism. HKUST-1 is relatively well studied in this regard; the measured conductivities ${ }^{18,27,33,35}$ are in agreement with DFT. ${ }^{27,35,67}$ These calculations predict that MOFs comprised of carboxylate-type linkers are insulators, as evidenced by the very flat and narrow bands (the total dispersion in the valence band is only $\sim 0.1 \mathrm{eV}$ ), indicating that there is little delocalization in spite of the presence of optical LMCT bands.

Theory can also provide a rational basis for metal ion selection to improve metal-linker overlap for through-bond conduction. Dramatic changes in conductivity were achieved by varying the metal ion in a thiol analogue of MOF-74. In this MOF, through-bond conduction and high mobility are enabled by infinite chains of (-Mn(II)-S-) linkages. ${ }^{50}$ However, the bands are again rather narrow, only $\sim 100 \mathrm{meV}$ and the activation energy for charge transport is high, $0.81 \mathrm{eV}$, indicating that weak hopping transport is the likely mechanism. Correlation effects are also likely responsible for the high activation energy, given the $\mathrm{Mn}$ (II) $\mathrm{d}^{5}$ configuration. In contrast, charge transport in the $\mathrm{Fe}(\mathrm{II})$ version of this MOF proceeds with an activation energy of only $0.28 \mathrm{eV}$, producing a conductivity that is six orders of magnitude higher than the $\mathrm{Mn}$ (II) compound. ${ }^{49} \mathrm{~A}$ similarly large conductivity difference is predicted for the Mn and Fe versions of MOF-74. Here, DFT calculations suggest that the difference results from the lower work function, higher charge density, and greater charge density in the Fe(II) MOF.

The first calculation of charge mobility in a MOF was reported by Musho and $\mathrm{Wu}$, who modeled the effect of linker functionalization on the electronic structure of the $\operatorname{Zr}(\mathrm{Iv}) \mathrm{MOF}$ UiO-66. ${ }^{62}$ The Boltzmann eqn (2) with a relaxation time approximation was used to compute the mobility:

$$
\mu=\frac{q \tau_{\mathrm{s}}}{m^{*}}=\left(\frac{C_{1} \hbar^{4} q}{E_{1} q}\right)^{2}\left(m_{\mathrm{e}} m^{*}\right)^{5 / 2}\left(T k_{\mathrm{B}}\right)^{3 / 2}
$$

in which $C_{1}$ is the elastic constant, $E_{1}$ is the three-dimensional deformation potential, $m^{*}$ is the effective mass of the charge carrier, $m_{\mathrm{e}}$ is the mass of an electron, $T$ is the temperature, and $k_{\mathrm{B}}$ is the Boltzmann constant. The predicted mobilities are not high - $\sim 10^{-3} \mathrm{~cm}^{2} \mathrm{~V}^{-1} \mathrm{~s}^{-1}$ - leading to conductivities of $\sim 4 \times 10^{-4} \mathrm{~S} \mathrm{~cm}^{-1}$. Consistent with these predictions are the rather flat electronic bands. However, a tunable band gap is predicted; the native MOF has a fairly wide gap of $3.10 \mathrm{eV}$, whereas the $\mathrm{NO}_{2}$-functionalized MOF a gap of only $2.20 \mathrm{eV}$. Together, these results demonstrate that both bandgap and charge density can be tuned by relatively straightforward linker functionalization, another encouraging sign that MOF synthetic versatility can lead to electronic materials with tailored properties.

The $\mathrm{Ni}_{3}(\mathrm{HITP})_{2}$ MOG discussed above demonstrates that the electronic conductivity of nanoporous MOFs can approach that of some organic conductors (Table 1). This stimulated several theoretical studies probing the mechanism of conductivity, possible alterations of the structure to achieve higher conductivity, and the potential for unusual electronic behaviors. Substitution of other metals for nickel is predicted to lead to a number of interesting properties. Replacing $\mathrm{Ni}$ (II) with $\mathrm{Cu}$ (II) can be used to modulate the strength of the metal-metal and $\pi-\pi$ interactions, leading to metallic conductivity in the bulk material and semiconductivity in a monolayer. ${ }^{68}$ As discussed above, Foster et al. proposed a different strategy to achieve semiconducting behavior in this structure, substituting $\mathrm{Cr}$ (III) ions for $\mathrm{Ni}(\mathrm{II})$ and inserting pillar ligands between the two dimensional sheets. ${ }^{14}$ More exotic effects have also been predicted. Zhou et al. predict that quantum spin Hall and $Z_{2}$ metallic states can be achieved in a monolayer of this material. ${ }^{69}$ Dong et al. subsequently predicted that substituting $5 \mathrm{~d}$ metals for the nickel ions and partial or complete replacement of the nitrogen atoms would lead to a quantum anomalous Hall effect, which to date has only been realized in a few materials. ${ }^{70}$ Thus far, only the bulk forms of the all-Ni and all$\mathrm{Cu}^{71}$ of these possible structures have been synthetically realized (reported film thicknesses are $\sim 100 \mathrm{~nm}$ ). These predictions are intriguing, however, and suggest that properties difficult to achieve by fully inorganic approaches may be accessible in MOFs. However, control of layer thickness likely will be essential; in particular, methods to form monolayers or a specific number of $2 \mathrm{D}$ layers must be developed. Some recently developed approaches to growing layered MOFs, ${ }^{72-74}$ including one in which monolayers of a porphyrin MOF were produced, ${ }^{75}$ are encouraging in this regard. Defects at layer edges, which may be crucial to topological insulator behavior, must also be controlled. Both are major challenges that have not been effectively addressed thus far.

\subsection{Ionic conductivity}

Ion conduction is a critical aspect in many energy storage and -conversion devices, for instance $\mathrm{Li}(\mathrm{I})$ and proton transport in Li-ion batteries and many fuel cell types, respectively. Cointegration of power functions directly on microelectronic chips is currently attracting a lot of interest for application in self-powered electronics such as portables, wearables and implants. To enable such applications, a suitable ionic conductor needs to be selected to serve as electrolyte. In macroscale 
Li-ion batteries, porous polymer sheets soaked in an organic $\mathrm{Li}(\mathrm{I})$ salt solution are typically used to fulfill this role. Nevertheless, replacing these systems with solid-state electrolytes is an active research topic, mainly because of their volatility, flammability and reactivity towards the electrodes. ${ }^{76}$ In the context of microelectronic devices, integration of liquid components as part of vertical stacks is not possible due to structural reasons and incompatibility with vacuum technology. Similarly, the control over relative humidity, needed for optimal performance of many proton conductors, might be harder to achieve in microelectronics than in larger equipment.

The regular arrangement of identical channels in a MOF framework hints at the possibility for efficient transport of ionic species. Most work in this area has focused on proton conduction. As illustrated in Fig. 5a, three general strategies can be identified to introduce mobile protons in the MOF pores: through counter ions as proton carriers, via the introduction of acidic functional groups on the framework or via the inclusion of protonated guest molecules (guest@MOF). The properties of resulting proton-conducting MOFs have been reviewed in-depth. ${ }^{77-79}$ Proton conduction in these materials typically occurs via a "vehicle mechanism" based on the diffusion the ionic carriers $\left(\mathrm{H}_{3} \mathrm{O}^{+}\right)$or the "Grotthuss mechanism" based on transfer between neighboring sites through hydrogen bonds. Either type of mechanism can be dominant in the different proton introduction strategies. Interestingly though, the Grotthuss mechanism is the more efficient one, as confirmed by the abnormally high mobility of protons in water compared with other ions for which vehicle-type transport dominates. ${ }^{80}$ While most of the proton-conducting frameworks require hydration, several promising strategies have been developed for anhydrous proton transport in MOFs. Interesting strategies are the inclusion of protic guest molecules in porous frameworks ${ }^{81-83}$ and the inclusion of partially protonated phosphate groups, mostly in non-porous layered coordination polymers. ${ }^{84,85}$

A smaller share of work targeted metal cation transport in MOFs, and has mainly focused on Li(I) in view of its potential application in solid-state batteries. For example, grafting of lithium alkoxide compounds on the coordinatively unsaturated metal cation sites in MOF-74 results in immobilization of the alkoxide, while leaving the $\operatorname{Li}(\mathrm{I})$ ions relatively free to move along the one dimensional channels of the MOF (Fig. 5b). ${ }^{86}$ After loading of the grafted material with additional Li(I) salt in carbonate solvent a solid electrolyte could be obtained in the form of a dry free-flowing powder. After pressing the powder into pellets, the bulk Li(I) conductivity at room temperature was $0.012 \mathrm{mS} \mathrm{cm}^{-1}$. This value is at least two orders of magnitude higher than a range of equivalent non-grafted MOFs, and on par with organic polymer solid electrolytes. A similar approach was applied for modification of UiO-66, which contains threedimensional channels, resulting in a bulk conductivity at room temperature of $0.018 \mathrm{mS} \mathrm{cm}{ }^{-1}$ in the absence of added free Li(I) salts. ${ }^{87}$ Interestingly, cation mobility was stimulated in the latter case by utilizing bulky (tert-butyl) aliphatic groups to shield the counter charge of the MOF coordinated alkoxide. Instead of the organic carbonates or ethers typically used in
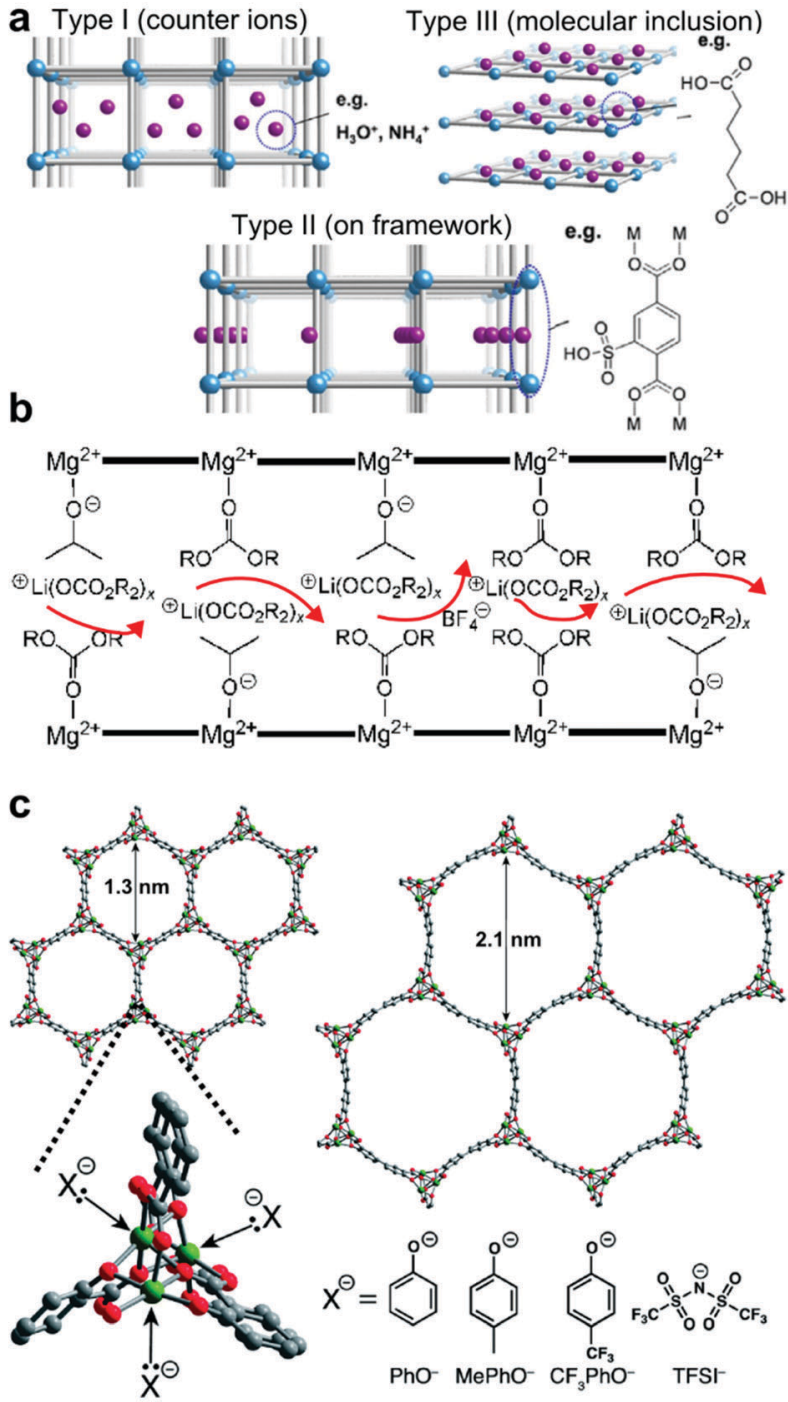

Fig. 5 Ion conduction in MOFs. (a) Different strategies for the introduction of acidic species as a source of protons in MOFs. Adapted from ref. 80. Copyright 2016 Wiley \& Sons. (b) Schematic representation of hopping transport of $\mathrm{Li}(\mathrm{I})$ in the alkyllithium grafted channels of MOF-74. Adapted from ref. 87. Copyright 2011 American Chemical Society. (b) Analogous method for $\mathrm{Mg}(\|)$ transport through grafting of phenolate and imidate on the inorganic node of MOF-74 and its larger pore isoreticular analogue. Adapted from ref. 89 .

Li(I) electrolytes, Kitagawa and co-workers included an ionic liquid in the MOF pores, thereby preventing its solidification. Interestingly, their results suggest that the Li(I) cations diffuse through the pores via the exchange of the solvating anions, similar to the Grotthuss mechanism in proton conductivity. ${ }^{88}$ Conduction of larger and/or multivalent metal ions imposes more challenging requirements on the development of solid electrolytes, for instance in charge compensation along the conduction path. Utilization of the permanent porosity in MOFs could be advantageous in this context. Analogous to the lithium alkoxide grafting approach, different magnesium phenolates and imidates were grafted on the coordinatively 
unsaturated metal cation sites in two isoreticular MOFs, MOF-74 and its expanded analogue which has a larger channel width (2.1 vs. $1.3 \mathrm{~nm}$ ) and lower surface polarity (Fig. 5c). ${ }^{89}$ The room temperature ionic conductivity of pressed powder pellets grafted with triethylene glycol dimethyl ether varied over four orders of magnitude for the different composites. The highest conductivity in the study $\left(0.25 \mathrm{mS} \mathrm{cm}^{-1}\right)$ was measured for the expanded material loaded with salts with non-coordinating anions. Notably, this conductivity is among the highest reported for $\mathrm{Mg}$ (II) solid electrolytes. ${ }^{90}$

\subsection{Energy harvesting and emission}

The hybrid organic-inorganic structure of MOFs provides numerous opportunities for light harvesting and tuning energy transport. Light can be absorbed by the linkers, metal cations, or guest molecules and transferred by exciton hopping or fluorescence resonance energy transfer (FRET; Fig. 3b). The same material characteristics that make MOFs attractive for charge transport operate here, namely synthetic tunability, long-range order and porosity as an additional design element. However, the use of MOFs as active components in lightharvesting devices was only discussed conceptually in the initial MOF roadmap. Since then, the field has progressed to the point that fundamental aspects of light harvesting in MOFs and their relationship to MOF design are beginning to be understood, and incorporation into devices has been achieved in a few cases. This body of work demonstrates the potential of MOFs for light harvesting, but also makes clear the major challenges that must be surmounted. Several reviews focused on synthetic approaches and post-synthetic functionalization, ${ }^{91,92}$ device aspects, ${ }^{93}$ and guest@MOF possibilities $^{21}$ recently appeared. Here we focus on the most recent device-related developments, in particular the possibility of using MOFs for photovoltaics (PV). The use of MOFs as emitter materials in light-emitting diodes is also receiving considerable attention, primarily from the point of view of emitter design, ${ }^{91,94}$ rather than device integration and performance.

Photovoltaics. MOFs can be envisioned as active materials in both the bulk heterojunction $(\mathrm{BHJ})^{95}$ and the dye-sensitized solar cell (DSSC) ${ }^{96,97}$ architectures. The advantages of MOFs in both cases are the synthetic versatility that would allow both light absorption and band alignment to be tuned, porosity that can be used to contain absorbers and/or charge carriers, and long-range order. In conventional $\mathrm{BHJ}$, an electron-donating semiconducting organic polymer serves the dual roles of light absorption and hole transport, with a molecular acceptor (often a fullerene) enabling exciton splitting and electron transport. The microstructure of these mixtures is tailored to have both ordered donor-acceptor regions to promote efficient exciton splitting and disordered regions of the electron- and hole-conducting components to improve conductivity. A MOF architecture emulating this would comprise a donor-acceptor pair using the MOF as one half of the junction and the pores as the host for a polymer or molecular semiconductor as the other half. ${ }^{98}$ This concept is yet to be realized as a device, due to the lack of semiconducting frameworks with appropriate band gap and band alignment with a charge donor or acceptor material. However, observation of energy transport by FRET $^{99}$ and longlived charge separation ${ }^{100}$ in guest@MOF systems have been demonstrated.

In contrast, in a DSSC, light absorption and charge transport are performed by different materials, making the material engineering much less complex than for $\mathrm{BHJ}$ solar cells. MOFs are well suited to serve as the dye (i.e. light harvesting) component of a DSSC by virtue of the ability to build structures with multiple light absorbers locked into a stable crystalline structure. Moreover, the porosity provides an additional design element by enabling another light absorber to be used, as well as access for the electrolyte needed to regenerate the dye. A recent report shows that even drop-cast MOF nanocrystals can act as the sensitizer in a Grätzel-type cell, ruling out the possibility that free linker chemisorbed to the $\mathrm{TiO}_{2}$ surface is the actual light absorber. ${ }^{101}$ When considering the required properties of the MOF, light absorption in the visible and near IR ( 400-900 nm) is clearly essential. Closed-shell metal ions such as zinc are preferred, as these minimize the opportunities for exciton quenching by fast decay to the ground state or the LUMO, rather than undergoing charge injection. Other necessary properties are compatibility with electrolytes such as $\mathrm{I}^{-} / \mathrm{I}_{3}{ }^{-}$and pores large enough to allow the electrolyte to come into close contact with the linkers. Effective visible light absorption is not sufficient, however. Proper alignment of the MOF LUMO and HOMO levels with the CB of $\mathrm{TiO}_{2}$ and the VB of the electrolyte are also critical for efficient photocurrent generation. Walsh and coworkers modeled the chemical potential of several well-known MOFs and showed that in most cases the alignment with the $\mathrm{TiO}_{2}$ CB is poor. ${ }^{56}$

Porphyrin-containing linkers in zinc-based frameworks are a logical choice for MOF-based sensitizers. ${ }^{73}$ However, their light absorption is primarily in the $400-500 \mathrm{~nm}$ region. Adopting strategies employed in the design of molecular sensitizers, the light absorption peak in a MOF can be shifted somewhat to the red by functionalizing the porphyrin ring with electron donors, as was done with diphenylamino groups. ${ }^{65}$ MOFs composed of dicarboxyphenylporphyrin (DCPP) linkers can exhibit very high charge carrier generation efficiencies, on the order of $10 \%{ }^{65}$ Linkers that absorb light further to the red have also been designed computationally; ${ }^{98}$ however, these have yet to be incorporated into a MOF. In another example of integrating a photoactive $\mathrm{MOF}$, $\mathrm{TiO}_{2}-\mathrm{MIL}-125$ composites were made in a single-step synthesis and integrated in $\mathrm{ITO} / \mathrm{TiO}_{2}-\mathrm{MIL}-125 /$ perovskite-graphite devices. ${ }^{102}$ Incorporation of MIL-125 resulted in an apparent photosensitization, raising the energy conversion efficiency from 2.5 to $6.4 \%$.

MOF analogues of classical ruthenium dyes are another light harvesting strategy being explored to provide absorption in the middle of the visible spectrum. ${ }^{103-105}$ Morris and coworkers adopted this strategy, incorporating ruthenium centers into a UiO-67 framework, and performed extensive photophysical characterization $^{103,106,107}$ of these materials and thin film growth. An especially promising aspect of this work is that MOF sensitization outperformed a device sensitized by the 
molecular ruthenium complex. It was speculated that efficient charge injection is possible because the $\mathrm{Ru}$ centers near the $\mathrm{TiO}_{2}$ interface intercept distant excitons via FRET. Long hopping distances have been reported in porphyrin- and BODIPY-based MOFs as well, ${ }^{108,109}$ suggesting that the ordered crystalline structure of MOFs is a key advantage. A chiral, indium-potassium MOF was reported as a co-sensitizer with a conventional ruthenium dye (N719) was also reported. ${ }^{110} \mathrm{PV}$ cells also have been constructed by depositing HKUST-1 on mesoporous $\mathrm{TiO}_{2}$, yielding a reasonable prototype efficiency $(0.26 \%)$ and a moderate improvement over a bare $\mathrm{TiO}_{2}$ ref. 111 . The ruthenium analogue of HKUST-1 gives even higher efficiency $(1.22 \%){ }^{104}$ However, the exact role of the MOF is unclear; heavy iodine doping was necessary in both cases to achieve the reported efficiencies, presumably to install acceptable electronic conductivity.

Whether the band gap in a MOF is direct or indirect also has a bearing on its potential for PV and light emission applications. Direct band gap semiconductors efficiently absorb light to create excitons (bound electron-hole pairs) that can be split to produce current in a PV cell. In contrast, light absorption is slow for indirect semiconductors, making them unattractive for light absorption unless very thick layers are used; similarly, they are of little use for light-emitting devices. For virtually all MOFs, the nature of the band gap has not been established, but can be predicted as for the Sr-based MOF discussed in Section 2.1. Liu et al. were the first to consider the nature of the band gap for a porphyrin MOF and concluded that their material is an indirect band gap semiconductor (Fig. 6a). ${ }^{52}$ Timedependent DFT calculations predict a bandgap of $1.94 \mathrm{eV}$ for the zinc-metallated porphyrin linker and GGA-DFT indicates some dispersion in the valence and conduction bands. However, this dispersion is so small $(\sim 3 \mathrm{meV}$ in the $\Gamma-Z$ direction, perpendicular to the porphyrin planes) that semiconductivity via an indirect band gap is unlikely. The resulting electron carrier mass, $9.6 m_{\mathrm{e}}$ ( $m_{\mathrm{e}}=$ electron mass), is more than an order of magnitude higher than molecular organic conductors such as rubrene, ${ }^{45}$ suggesting that the electronic conductivity also will be poor. From a structural standpoint, through-space conduction between the adjacent porphyrin rings will be low, due to the $0.6 \mathrm{~nm}$ inter-ring separation. The zinc-carboxylate linkages between porphyrins are not expected to provide significant through-bond conductivity, as also indicated by the limited efficiency of initial explorations in PV using MOF-5 $5^{112}$ and $\mathrm{Al}_{2}$ (BDC) ${ }_{3}{ }^{113}$ Unfortunately, the temperature-dependent electrical conductivity was not measured, which could confirm this conclusion. In spite of these uncertainties, the thorough spectroscopic and device characterization performed by Liu et al. provide important data that encourage further research to design MOFs for light harvesting and photonic applications.

In addition to light absorption and/or carrier separation and transportation, MOFs are being explored for different roles in PV applications as well. For example, ZIF-8 films incorporated in dye-sensitized $\mathrm{FTO} / \mathrm{TiO}_{2}$ half-cells formed an energy barrier preventing interfacial charge recombination (Fig. $6 \mathrm{~b}$ and c). ${ }^{114}$ However, as indicated by the decreased energy conversion efficiency (from 5.11 to $4.31 \%$ ), this effect also hampers charge injection from the dye molecules to the semiconductor. In a follow-up study, this unfavorable side-effect was to a certain extent overcome by modifying the fabrication protocol and
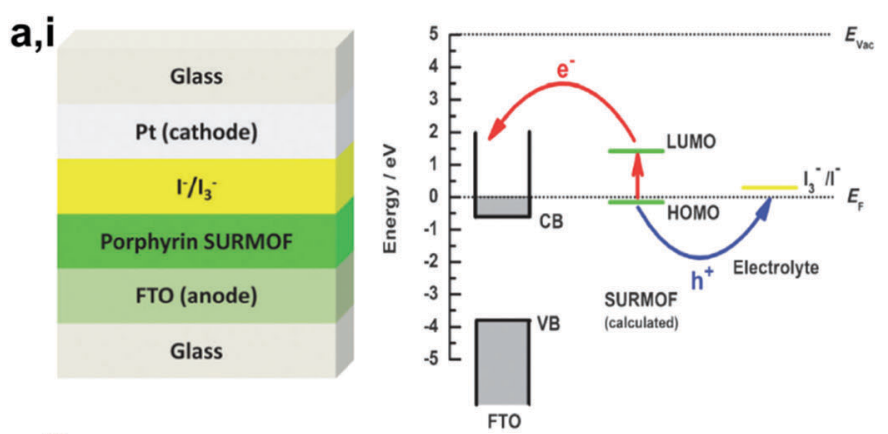

a,ii

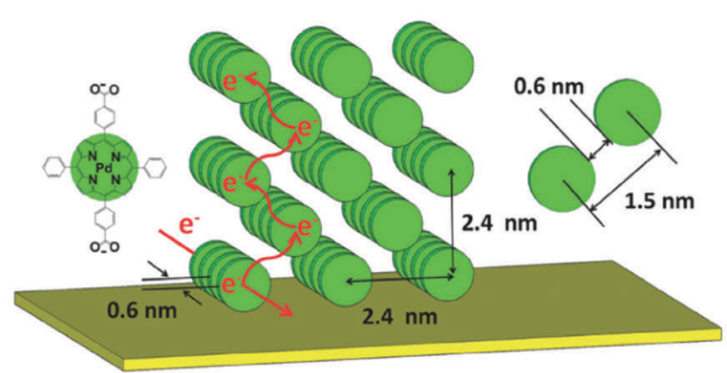

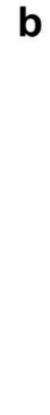

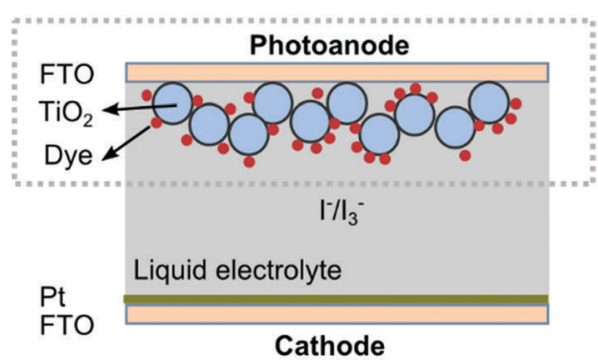

C

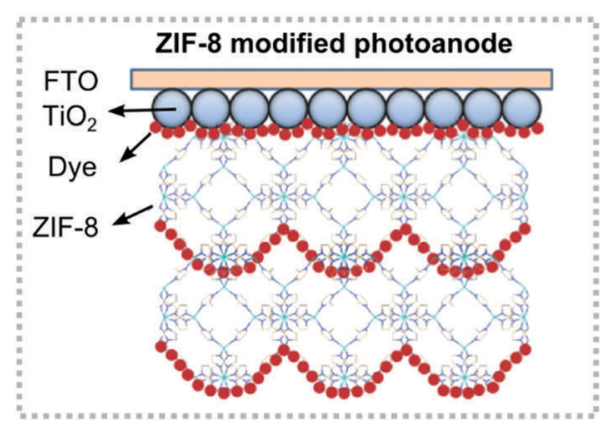

Fig. 6 Integration of MOFs in photovoltaic research. (a,i) FTO/SURMOF/ $\mathrm{I}^{-}-\mathrm{I}_{3}^{-}$/Pt PV cell assembly. The energy diagram shows photon absorption and exciton separation in the porphyrin SURMOF. (a,ii) Periodic nematic stacking of porphyrin linkers in the SURMOF (metal nodes are omitted for clarity). A proposed exciton diffusion pathway is shown by the red arrows. Adapted and reproduced with permission from ref. 52 . Copyright 2015 Wiley $\&$ Sons. (b) Design of a typical DSSC. (c) MOF as barrier layer for immobilization of dyes to $\mathrm{TiO}_{2}$ at the photoanode half-cell. Adapted from ref. 115 . Copyright 2015 Wiley \& Sons. 
depositing the ZIF-8 barrier layer on top of pre-adsorbed dye molecules, thereby increasing the energy conversion efficiency to $>6 \%{ }^{115}$ Recent work also suggested a role for MOFs in wet chemical fabrication of organic-lead halide perovskite devices, one of the fastest growing PV technologies. As the perovskite unit cell is significantly smaller than that of most MOFs, nucleation of perovskite nanodomains should be possible within a host with large enough pores. This strategy was demonstrated by adding nanocrystals of the Zr-based MOF$525\left(1.8 \mathrm{~nm}\right.$ pore size) to a perovskite spin coating solution. ${ }^{116}$ Surprisingly, the addition of the MOF caused a significant increase in the efficiency (from $10.1 \%$ to $12.0 \%$ ) that seems related only to the MOF scaffolding during perovskite nucleation.

Light-emitting devices. Besides PV applications, the designer nature of MOFs offers opportunities in light emission as well. Many frameworks are luminescent and through the combined engineering of inorganic nodes, organic linkers and guest space and therefore offer more design parameters than alternative materials in this area. Examples of approaches to tune the emission color include fine-tuning the linker ${ }^{91,94,117}$ or the metal ion $^{118}$ (especially lanthanide ions ${ }^{119}$ ) alone or in combination, or alternatively, by infiltrating the pores with luminescent species ${ }^{120,121}$ or by creating MOF nanoparticles. ${ }^{122}$ These materials are primarily targeted for use as phosphors that down-convert the emission of UV or blue LEDs to longer wavelengths and create broad spectrum white light emission. Due to the rapidly growing market for LEDs, a search is ongoing for efficient phosphors that are low-cost, sustainable, safe, nontoxic, and easily processible. Such materials can become alternatives for the rare-earth phosphors originally developed for fluorescent lighting and now implemented in LEDs.

One of the earliest studies of emission-tuning in MOFs for phosphor applications was the synthesis of $\mathrm{In}_{2} \mathrm{BTB}_{3}$ by Nenoff and co-workers, and the observation of its inherent broad-band white emission when excited at $330 \mathrm{~nm} .{ }^{123}$ It was shown that the emission color temperature, important for practical warm white-light applications, could be rationally tuned by doping the framework with isomorphic red-emitting Eu(III). However, the internal quantum yield of the proof-of-concept system, $4.3 \%$, was still extremely low. A great deal of progress has been made since then, most prominently by $\mathrm{Li}$ and co-workers. ${ }^{124}$ First, they showed that the photoluminescent properties of the host frameworks and selected guests can be rationally combined to improve the guest@MOF composite material performance. For example blue-emitting Cd-TATPT was prepared with an optimized concentration of a yellow-emitting cationic Ir(III) complex encapsulated in the pores $(20 \%$ internal quantum yield for excitation at $370 \mathrm{~nm}$ ). ${ }^{125}$ An interesting iteration of this concept is the one-step synthesis of MOF-based hostguest systems. Tan and co-workers demonstrated how a $\mathrm{Zn}$ based emitter can be encapsulated during the formation of ZIF-8, leading to significantly enhanced luminescence stability. ${ }^{126}$ DFT moreover indicates cooperativity via a host-guest charge transfer mechanism. A second important concept is the inhibition of non-radiative decay via chromophore rotation, vibration, and torsion by anchoring such emitters into the rigid MOF
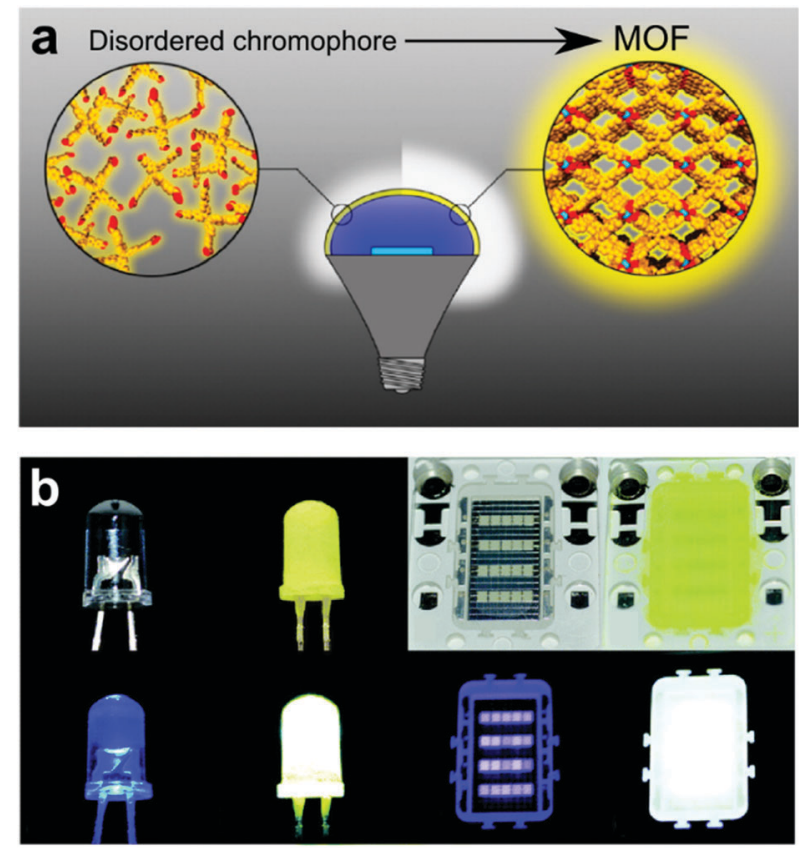

Fig. 7 MOFs for light emitting devices. (a) Schematic illustration of the improvement in quantum yield of a yellow-emitting organic chromophore by incorporation in a rigid metal-organic framework. Adapted from ref. 124. Copyright 2016 American Chemical Society. (b) Blue LED bulb and plate coated with solution-processed $Z n_{2}$ (TCPBE) MOF phosphor as a demonstration of application in white LEDs. Adapted from ref. 127.

backbone (Fig. 7a). For example, tetrakis(4-carboxyphenyl)ethylene $\left(\mathrm{H}_{4} \mathrm{TCPBE}\right)$ was used as a linker in the synthesis of a $\mathrm{Zn}_{2}(\mathrm{TCPBE})$ framework. ${ }^{127}$ Red shifting of the emission spectrum was observed through incorporation in the MOF, as well as an increase of the internal quantum yield from $<50 \%$ for the organic by itself to $>80 \%$ for the MOF. Third, by incorporation of a band gapmodulating co-ligand, the internal quantum yield can be further enhanced, up to $90.7 \%$ at $400 \mathrm{~nm}$ excitation for $\mathrm{Zn}_{6}(\mathrm{BTC})_{4}(\mathrm{TPPE})_{2}$ $\left(\mathrm{TPPE}=1,1,2,2\right.$-tetrakis(4-(pyridin-4-yl)phenyl)ethylene). ${ }^{128}$ The above results demonstrate that the incorporation of tailored photoluminescent linkers in MOFs can be used as a combined strategy for immobilization, stabilization and fine-tuning of their emission characteristics. Powder coating of blue-emitting LEDs with MOF phosphors has been demonstrated by several groups as a devicelevel proof-of-concept (Fig. 7b).

In contrast to MOF coatings on the outside of a LED, deeper integration of light-emitting MOFs as an active component, i.e. as the actual emitting layer in which electron-hole recombination results in light emission, would avoid the energy losses intrinsic to down-conversion and could enable new emission signatures and eliminate or reduce the cost of certain critical elements. Nevertheless, integration of (semi)conducting MOFs in LEDs has thus far not been reported. To achieve this goal, the MOF must be both emissive in the desired spectral region and have good charge mobility. A low density of electrically active defects and high charge mobility are also essential for an efficient LED so that injected holes and electrons can penetrate the emitting layer(s) and recombine to form excitons with 
minimal non-radiative recombination. As discussed in Section 2.1, measurements of electronic mobility in MOFs are rare. ${ }^{8}$ In the few cases for which they are known, the frameworks are comprised of transition metal cations that are likely to be only weakly luminescent, if at all. Mobilities have been reported for an indium-isophthalate framework ${ }^{129}$ and for a zinctetrathiafulvalene $\mathrm{MOF},{ }^{22}$ but the luminescence spectra were not reported. Clearly, this is a promising area that is ripe for new research and in which the ability to build multiple functions into the framework is an important advantage.

\subsection{Magnetic properties}

The presence of open-shell metal centers can impart magnetic properties in MOFs. Several reviews discussed such structureproperty relationships and the magnetic changes that occur in response to different chemical and physical stimuli. ${ }^{130,131}$ Therefore, in this section we focus on important progress towards understanding guest-induced spin crossover, the only magnetic property of MOFs that has received significant attention from a device point of view. Spin crossover occurs when the spin state of a metal ion switches from the low-spin to the highspin ground state electronic configuration and vice versa. ${ }^{132}$ The resulting changes in occupation of the antibonding and nonbonding molecular orbitals modulate the magnetic and optical properties of the material, providing a transduction mechanism for sensing near room temperature. ${ }^{133}$ The spin transition temperature and thermal hysteresis are two related properties that can be modulated by altering the ligand field strength and cooperativity that arises from intermolecular interactions in the complex. ${ }^{134}$ Although spin crossover can also be induced by physical stimuli such as light, pressure, and temperature, we focus here on guest-induced effects. Given the wide array of binding motifs and chemical functionalities in MOFs, there are numerous opportunities for tuning spin crossover behavior, including the ability to exploit porosity for guest@MOF interactions not possible in traditional spin crossover complexes. ${ }^{40}$

The first account of spin crossover in a porous MOF was reported by Halder et al., who demonstrated that the Fe(II) spin crossover center in $\mathrm{Fe}_{2}(\mathrm{AZPY})_{4}(\mathrm{NCS})_{4}$ (AZPY = trans-4,4'azopyridine; NCS = thiocyanate) renders the framework responsive to various guest molecules. ${ }^{135}$ While spin crossover is absent for the evacuated framework, modulated spin crossover is achieved in the presence of several alcohols, thereby illustrating the potential for reversible sensing. Prior to this work, spin crossover effects due to guest counter ions were demonstrated in three-dimensional networks such as the $\left[\mathrm{Fe}(\mathrm{BTR})_{3}\right]\left(\mathrm{ClO}_{4}\right)_{2}$ (BTR $=4,4^{\prime}$-bis-1,2,4-triazole) system, ${ }^{136}$ but these materials are not stable upon guest removal. Halder, Kepert and co-workers later extended their initial study by replacing AZPY with different bis-pyridyl ligands to further tune guest-induced spin crossover behavior. ${ }^{137-140}$ Following these seminal findings, several groups have exploited three-dimensional Hofmann clathrates as a tunable platform for probing spin crossover effects. These materials have a $\mathrm{Fe}(\mathrm{L})\left[\mathrm{M}(\mathrm{CN})_{4}\right]$ structure, in which $\mathrm{L}$ is a pillaring ligand between the $\mathrm{Fe}(\mathrm{II})$ cations and $\mathrm{M}$ is $\mathrm{Ni}(\mathrm{II}), \mathrm{Pd}(\mathrm{II})$ or $\mathrm{Pt}(\mathrm{II})$. A summary of these structures and various guest-induced effects can be found in recent reviews. ${ }^{141,142}$ For sensing applications, the $\mathrm{Fe}$ (pyrazine) $\left[\mathrm{Pt}(\mathrm{CN})_{4}\right]$ Hofmann variant is likely the most promising as spin crossover is observed near room temperature in the bulk form as well as in thin films. ${ }^{143-145}$

A number of recent advances bode well for the development of MOF-based magnetic sensing devices. While initial spin crossover studies focused primarily on small, polar molecules, the range of guest species targeted now spans an array of solvent and gas molecules, including halogens and aromatic species. ${ }^{141,142}$ Recent studies indicate that weakly interacting molecules such as nitrogen and oxygen cannot yet be detected using spin crossover. However, among the more strongly interacting species that can be detected, the current understanding is too limited to confidently predict their spin crossover effects. Molecular modeling is playing an increasingly important role in elucidating the underlying principles governing the spin crossover response, with recent studies of the $\mathrm{Fe}($ pyrazine $)\left[\mathrm{Pt}(\mathrm{CN})_{4}\right]$ framework providing valuable insight into the origin of its guestmodulated behavior. ${ }^{146,147}$ For example, in agreement with experiment, ligand field molecular mechanics simulations predict that the spin crossover temperature of $\mathrm{Fe}($ pyrazine $)\left[\mathrm{Pt}(\mathrm{CN})_{4}\right]$ decreases with increasing water loading, a phenomenon attributed to the elongation of the Fe(II)-pyrazine bonds caused by water interactions. ${ }^{147}$ In addition to the successful growth of spin crossover MOFs as thin films, ${ }^{143-145,148-150}$ recent work demonstrated reversible ppm-level gas sensing by monitoring refractive index changes upon aromatic volatile organic compound adsorption in a spin crossover $\mathrm{Fe}(\mathrm{BPAC})\left[\mathrm{Pt}(\mathrm{CN})_{4}\right](\mathrm{BPAC}=$ bis(4-pyridyl)acetylene) material. ${ }^{151}$ What is still missing from these developments is the demonstration of a MOF-enabled sensing device whereby a spin crossover MOF is incorporated as an integral component of the device hardware.

\subsection{Dielectric properties}

Dielectric polarization. The charge carriers of nonconductive materials, by definition, cannot move freely along the direction of electric fields. Nonetheless, these carriers can be locally displaced from their equilibrium position by an electric field, leading to dielectric polarization (e.g. in the medium in between two capacitor electrodes). The dielectric constant $(\kappa)$ is the resistance of a medium to the formation of an internal electric field. When $\kappa$ is decreased, the resistance to formation of an electric field in the medium will increase (maximum resistance at $\kappa=1$ in the absence of any medium: vacuum). For a given material, $\kappa$ is described by the Debye eqn (3), in which $N$ is total density of dipoles, $\alpha_{\mathrm{e}}$ the electronic polarization, $\alpha_{\mathrm{d}}$ the distortion polarization, and the third term corresponds to the thermally averaged contribution of the permanent dipole $\mu:^{152}$

$$
\frac{1-\kappa}{\kappa+2}=\frac{4 \pi}{3} N\left(\alpha_{\mathrm{e}}+\alpha_{\mathrm{d}}+\frac{\mu^{2}}{3 k T}\right)
$$

Hence, the three terms in the Debye equation represent the fieldinduced displacement of electron clouds, the displacement of nuclei or ions, and the orientation of permanent dipoles. In general, $\kappa$ decreases with decreasing material density, the introduction of porosity, and less polarizable atoms and bonds. 

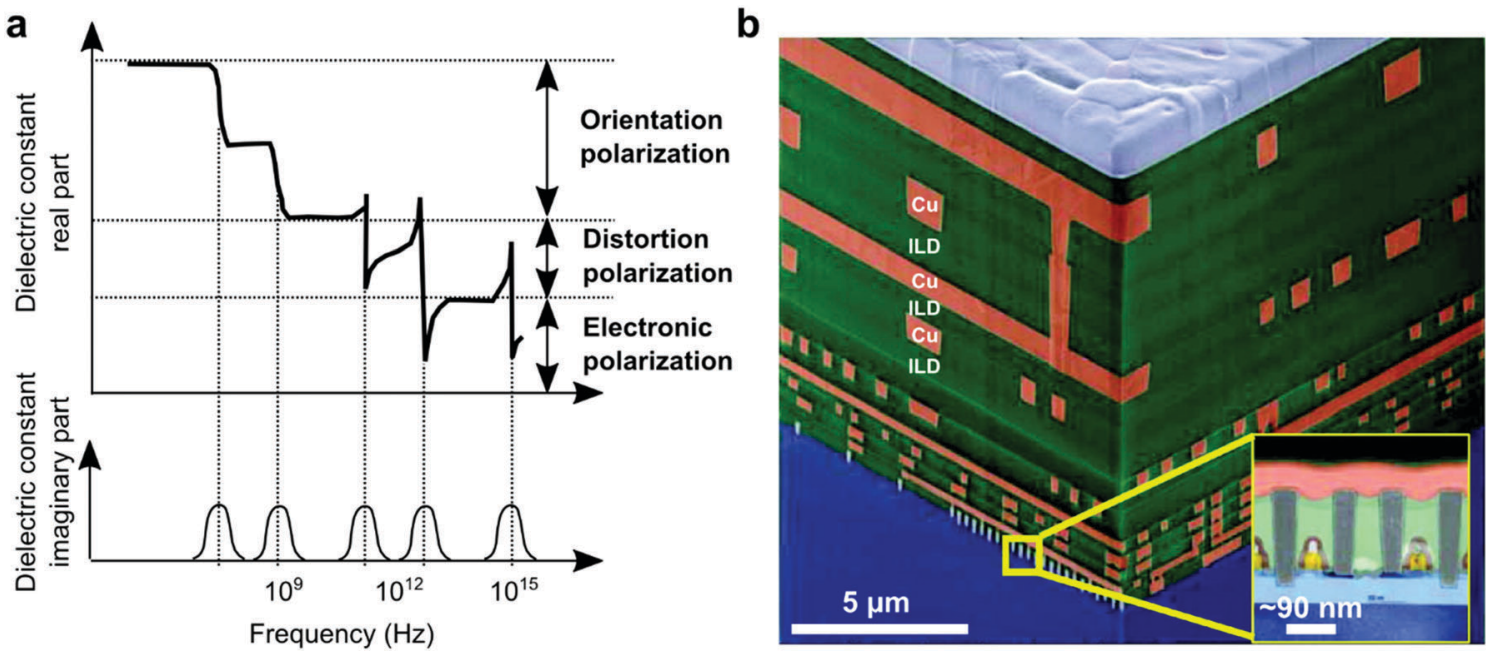

Fig. 8 MOFs as low $-\kappa$ dielectrics. (a) Simulated plot of the real and imaginary parts of the dielectric constant as a function of frequency for a fictional material. Redrawn from ref. 153. (b) False-color cross-sectional electron microscopy image of $90 \mathrm{~nm}$ CMOS microprocessor. Interconnects (red-brown) are embedded in multiple layers of low- $\kappa$ ILD (green). The inset shows the transistor layer that is position at the bottom of the vertical stack. Adapted from ref. 152. Copyright 2010 American Chemical Society.

Importantly, the different contributions to polarization are frequency-dependent, i.e. they are associated with different frequency regimes based on the speed at which the induced polarization is able to adjust to an applied alternating electric field. Electron clouds, being lowest in mass, contribute to the polarizability even at frequencies as high as $10^{15} \mathrm{~Hz}$. Ions and molecular dipoles, by contrast, will only resonate below ca. $10^{12} \mathrm{~Hz}$ and $10^{9} \mathrm{~Hz}$ or lower, respectively (Fig. 8a). ${ }^{153}$ Typical operation frequencies in integrated electronic circuits are in the range of $10^{9} \mathrm{~Hz}$. This means that all three Debye terms can contribute to the $\kappa$, and therefore should be taken into account in the design of suitable dielectrics.

Adsorption and desorption of guest molecules in MOFs leads to variation of the density and polarizability and a related transition in dielectric constant, from $\kappa_{(\mathrm{MOF})}$ to $\kappa_{\text {(guest@MOF). }}$. For example, by replacing less polarizable guest molecules with more polarizable counterparts, the $\kappa(60 \mathrm{~Hz})$ of Ni(II)-bisbenzimidazole coordination polymers increases from 4.8 to $12.6 .{ }^{154}$ Maxwell's eqn (4) relates the refractive index (n) to the real part of the dielectric constant at the frequency of optical electromagnetic waves (e.g. $10^{15} \mathrm{~Hz}$ for visible light):

$$
n=\sqrt{\kappa}
$$

Hence, guest-induced variation of polarizability is not only a signal transduction route for electronic chemical sensors, but also for optical chemical sensors $\left(n_{\text {(MOF) }}\right.$ to $\left.n_{\text {(guest@MOF) }}\right)$. An important distinction lies in the fact that orientation and distortion polarization effects do not contribute to the optical signal, whereas they do in electrical transduction at lower frequencies. Related chemical sensing approaches that have been reported in literature will be discussed in the in Section 4.2.

Low- $\boldsymbol{\kappa}$ dielectrics. Dielectric materials play a pivotal role in microelectronics, as the capacitance is one of the fundamental properties of a circuit. In integrated circuits, different metal interconnects are separated by an interlayer dielectric (ILD, Fig. 8b).
Downsizing of CMOS technology requires increasingly challenging ILD properties. For example, the parasitic (i.e. unwanted) capacitance between neighboring interconnects has to be kept to a minimum as it hampers signal propagation speed and increases power dissipation. To address this issue, reduced $\kappa$ materials have to be implemented. By definition, low- $\kappa$ dielectrics are materials that are less polarizable than $\mathrm{SiO}_{2}$, or $\kappa<4$. Porous materials are of particular interest as the incorporation of open space lowers the density $(N)$ and therefore $\kappa$. However, at the same time the pore aperture should be kept to a minimum to prevent metal penetration and electromigration during fabrication and device operation. Hydrophobicity is another requirement, as uptake and entrapment of humidity need to be avoided $\left(\kappa_{\mathrm{H}_{2} \mathrm{O}}=\sim 80\right)$. Selection criteria such as processability and integration routes, mechanical properties (e.g. fracture properties and thermomechanical loading), breakdown voltage and environmental impact are also decisive for the selection of future ILD materials. ${ }^{155}$ The development of $\kappa<2.4$ materials has been ongoing for years, and selecting suitable candidates for $\kappa<2.0$ has been particularly challenging (e.g. impact of high porosity on processability, resistance to processinduced damage and mechanical stability). Nanoporous materials based on modified $\mathrm{SiO}_{2}$ (e.g. porous, carbon-doped and fluorinated $\mathrm{SiO}_{2}$ ) are now commercially implemented. Although further improvement will depend on finding replacements, the difficulty of this search is evident and resulted in postponing with every edition of the ITRS roadmap in the last decade. ${ }^{156}$

The potential of MOFs for low- $\kappa$ applications was initially put forward by Zagorodniy et al. through theoretic evaluation of the (static) $\kappa$ of a range of different $\mathrm{Zn}$ (II)carboxylate IRMOFs. ${ }^{157}$ The semi-empirical Clausius-Mossoti formula was used; neglecting orientation and distortion polarization contributions. In a later study, these estimations were confirmed by first-principle DFT calculations, showing only a slight underestimation $(<10 \%){ }^{158}$ Above all, these calculations suggest that reticular MOF design can be utilized as a route to $\kappa<2.0$ materials with 
desirable band gaps ( $>5 \mathrm{eV}$ ) and suitable mechanical properties. However, experimental benchmarking of the theoretically proposed materials requires synthesis of the proposed materials as high-quality thin films in metal-insulator-metal (MIM) capacitors for analysis by impedance spectroscopy. This has not yet been achieved for all highly porous IRMOFs that were proposed. $\mathrm{Lu}$ and co-workers, in a recent review, summarized the handful of studies reporting experimental low- $\kappa$ behavior of MOFs at relevant frequencies. ${ }^{159}$ In line with the requirements for low- $\kappa$ applications, the authors indicate that while MOFs show potential, efforts are needed to develop suitable integration strategies and computational screening. Currently, a few cases have been reported that can be considered particularly promising. Eslava et al. reported $\kappa=2.3(0.1 \mathrm{MHz})$, for the prototypical Zn(II) framework ZIF-8. ${ }^{160}$ The ZIF subclass of MOFs are prominently utilized in thin film studies, and many compatible integration routes either available or in development (see Section 3.3). In agreement with the low density of ZIF-8 $\left(0.95 \mathrm{~g} \mathrm{~cm}^{-3}\right)$, the distortion contribution to $\kappa$ was found to be low $(0.5$, in comparison with 1.9 for $\mathrm{SiO}_{2}$ ). Hence, the observed $\kappa$ was only slightly higher than the Clausius-Mossoti calculated (static) $\kappa=1.98$. In addition, the breakdown voltage $\left(>2 \mathrm{MV} \mathrm{cm}^{-2}\right)$, leak current $\left(10^{-8} \mathrm{~A} \mathrm{~cm}^{-2}\right.$ at $\left.1 \mathrm{MV} \mathrm{cm}^{-2}\right)$, environmental stability and hydrophobicity of the ZIF-8 thin films were roughly within the specifications needed for future low- $\kappa$ materials. Usman et al. reported $\kappa=2.4(0.1 \mathrm{MHz})$, for two-dimensional $\operatorname{Sr}(1,3-\mathrm{BDC})$ measured as pressed powder pellets. ${ }^{161}$ On the one hand, these experimentally confirm the potential predicted for similar metalcarboxylate MOFs. On the other hand, interstitial gaps and interfaces in the pressed pellets could interfere with the data interpretation and further work is needed to benchmark these materials. Wöll and co-workers studied the optical constants of thin HKUST-1 films by spectroscopic ellipsometry at $750 \mathrm{~nm}$, or $10^{14} \mathrm{~Hz} .{ }^{162}$ After full dehydration of the framework $n=1.39$ was measured, which is in relatively good agreement with the Clausius-Mossoti predicted (static) $\kappa=1.7(n=1.30)$. Importantly, the optical constants do not include lower frequency contributions and also in this case MIM capacitors need to be fabricated and electrically analyzed. In general, further study in this area is needed, particularly since MOFs can offer unexplored strategies to further minimize $\kappa$ (e.g. isoreticular synthesis using longer linkers to realize even lower density, low polarizability fluorinated frameworks).

High- $\boldsymbol{\kappa}$ dielectrics. Materials that combine high- $\kappa$, low electrical leakage and high breakdown voltage are needed in microelectronics to enable capacitive coupling. For example, the gate dielectric of a downsized microprocessor FET needs to be high- $\kappa$, particularly when fast switching and low leakage is required. Currently, metal oxides such as $\mathrm{ZrO}_{2}$ and $\mathrm{HfO}_{2}$ are implemented as high- $\kappa$ dielectrics. However, organic electronics require alternative materials that couple suitable dielectric properties with e.g. lower mechanical brittleness. ${ }^{163,164}$ Because of the versatile building blocks that can be incorporated in the MOF backbone and pores, their use has been proposed in this context. In a recent study by $\mathrm{Li}$ et al., electrochemically grown thin films of the 2-fold interpenetrated $\mathrm{Zn}$ (dimethylammonium)(TBTC) framework, MOF-246 (TBTC = 1,3,5-tris[4-(carboxyphenyl)oxamethyl]-2,4,6-trimethylbenzene), demonstrated $\kappa=19.5$, at $10^{6} \mathrm{~Hz} .{ }^{165}$ The high $\kappa$ value was attributed to the close-packed interpenetration and electronic interaction of the frameworks and its trapped solvent guests (dimethylformamide and ethanol). The film exhibited passable high- $\kappa$ insulating behavior, showing a leakage current of $10^{-7} \mathrm{~A} \mathrm{~cm}^{-1}$ at $1 \mathrm{kV} \mathrm{cm}^{-1}$ and a breakdown voltage above $10 \mathrm{kV} \mathrm{cm}{ }^{-1}$. However, a significantly smaller $\kappa=5.9$ was observed for a powder layer of the same material, possibly indicating better aligned space-charge distribution in the monolithic film. Yet, further study is needed to understand this phenomenon. Connected to the previous examples, non-centrosymmetric orientation of (induced) dipoles in MOFs and guest@MOF approaches can give rise to ferroelectric (high- $\kappa$ ) dielectric behavior, i.e. spontaneous polarization that can be electrically switched in a reversible way. The progress and perspectives in this burgeoning field have been discussed by specialized reviews. ${ }^{166,167}$

\section{Microfabrication and current state of MOF processing}

The practical application of functional materials in devices requires synthesis methods that provide control over properties and morphology. In addition to performance, durability and cost as criteria to assess a material, processability and compatibility with all fabrication steps is key. In the next sections, a general introduction to typical microfabrication steps is followed by a discussion on the status of MOF processing in this context. The latter includes thin film deposition and patterning, templating uses of MOFs and mechanical thin film behavior to illustrate the importance of implementation-related engineering and its relation with fundamental materials properties. Note that a generalized description of microfabrication is inevitably an oversimplification, and that production routes different from the example of CMOS technology are utilized in specific areas such as large-area electronics, low-cost devices on plastics, etc.

\subsection{Microfabrication: key characteristics and processing steps}

CMOS production of integrated circuits (ICs, chips) is an excellent example of cost-effective industrial microfabrication as its persistent progress enabled modern information technology. ${ }^{168}$ Moreover, microfabrication techniques and infrastructure have become essential to science and technology areas far beyond semiconductor technology, e.g. in nanoscience, at the interface between physics, biology and chemistry. Many upcoming or recently commercialized microelectromechanical systems, microfluidics, on-chip bioassays, etc. would not have been feasible without microfabrication routes that enable massively improved performance, fabrication costs, power requirements, and device reliability. ${ }^{169}$ In order to bring MOFs to the area of device research and development, it is essential to capitalize on this technology platform.

Microfabrication relies on sequences of the following unit operations: thin film deposition, patterning, doping and 


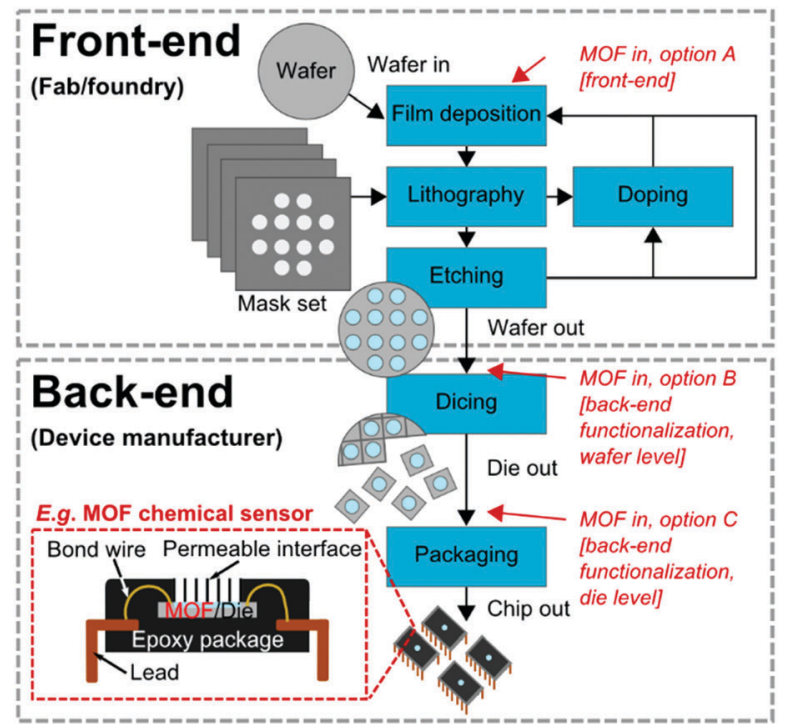

Fig. 9 Unit operations of microfabrication and chip production logistics. Three options for MOF integration during the production of a hypothetical CMOS fabricated chemical sensor are proposed: in the course of the microfabrication process (option A), before dicing step in back-end processing (option B) and on individual dies before packaging (option C).

etching (Fig. 9). Accordingly, electronic chips are generally constructed bottom-up, layer-by-layer and part-by-part, through vertical stacking of different materials. Integration of each material starts with deposition of a thin film on the substrate (or on the structure resulting from the previous sequences). A light-sensitive polymer, or photoresist, is then deposited on top of the layer and patterned through ultraviolet lithography. The pattern is transferred from the photoresist to the material through an etching process. Lastly, the remaining photoresist is removed from the assembly and the next sequence is initialized. For semiconductor devices, spatial-selective doping steps are included at different instances throughout the sequence. Whereas the above brief description is the textbook example of IC microfabrication, in reality many variations are utilized for case-specific causes. ${ }^{168}$ Markedly, well-established technology platforms now exist for a range of substrates beyond standard silicon wafers, such as glass and quartz (optical transparence, piezoelectricity), ceramic materials (chemical inertness, mechanical stability) and even organic polymers (flexibility and low-cost for large-area applications).

Deposition. Device fabrication critically depends on the deposition of homogeneous and pinhole-free films. Among a wide range of film growth techniques, vapor phase depositions are typically the process of choice, particularly for inorganics. ${ }^{170}$ These methods are categorized as either physical vapor deposition (PVD, i.e. direct deposition of an evaporated or sputtered material) or chemical vapor deposition (CVD, i.e. reaction of evaporated chemical precursors). A particularly flourishing CVD process is atomic layer deposition (ALD), in which alternated self-terminating reactions are used to enable bottom-up deposition of highly conformal films with precise thickness control. ${ }^{171}$ In addition, spin coating, spray coating and solution deposition are popular alternatives, particularly for organics. Many factors need to be taken into account in the development and selection of MOF thin film processing routes: control over composition and microstructure (crystallinity, epitaxy, grain size, grain boundaries, etc.), surface texture (low roughness), deposition speed, film-substrate interface chemistry and adhesion and compatibility of the deposition conditions with general prerequisites (avoidance of solvents, corrosive metal salts, chemical or physical contamination, etc.). ${ }^{172}$ In the context of inorganic microelectronic devices, MOFs can be considered relatively delicate in terms of chemical, mechanical and thermal stability, potentially leading to issues in other processing steps. Nevertheless, the type and number of steps following MOF deposition will depend on the position and integration level of the material and therefore its role, e.g. as embedded interlayer dielectric versus a chemical sensor coating in contact with the environment. If required, a device can be optimized to position the integration of a more delicate but critical material towards the back-end of the processing line (Fig. 9). In those cases, the deposition of that material cannot corrode or contaminate any of the previously deposited features. Note that in the cases when back-end processing is not possible (e.g. transistors), much more rigorous requirements are imposed upon these films but acceptable deposition conditions span a broader range.

Patterning, planarization and doping. Microscale patterning is the fundamental step that enables integration of complex functions in electronic devices. Basically, in this fabrication step the computer-assisted design of the device is transferred to the physical reality. Electron beam lithography is typically used to directly write a set of masks, which are subsequently used for mass-scale transfer of the patterns via photolithography. After selective removal of the photoresist, the functional material is patterned through etching. Wet etching, using liquid chemicals, as well as dry etching, using gas-phase chemistry, are frequently used. Additive patterning routes are a valuable alternative to the previous scheme. For example, area-selective deposition can be used to circumvent exposing materials to etching procedures (e.g. through shadow-mask deposition, soft lithography and microcontact printing, or lift-off lithography). Related to wet etching is the practice of chemical-mechanical planarization (CMP), which is utilized to smooth non-planar films in preparation of a next deposition step. This step is an essential part of current "Damascene" processing of the metal interconnects and ILDs in the fabrication of ICs. ${ }^{152,168}$ Similar to shadow-mask deposition of a material, the carrier type and carrier concentration of a semiconductor film can be altered in a spatially defined way through patterned doping. For inorganic semiconductors, this is achieved via ion beam implantation or solid diffusion of chemical dopants.

Back-end processing. After completion of all processing steps, the wafer substrate is diced to allow individual packaging of the chips that were produced in parallel. In this stage, advanced chip stacks can be fabricated through vertical bonding of different substrates (e.g. "flip-chip" assemblies), although integrating all functions on a single substrate is preferred. The role of packaging is to protect and preserve the performance of 
the device. In addition, the package provides larger-scale electrical contacts to the chip, for interfacing with a printed circuit board. Although crucial to enable devices, this engineering area is not discussed further here.

\subsection{Depositing and patterning MOF thin films}

The first step toward the fabrication of devices requires the deposition of MOF films, with control over composition, homogeneity, thickness, roughness and ideally crystal orientation. ${ }^{173}$ A second and similarly prominent aspect to fully exploit MOFs in the field of miniaturized devices requires the ability to fabricate MOF patterns from the micro- to the nano-scale. Since the publication of several reviews on fabrication methods for films and patterns, ${ }^{174-176}$ the field has further expanded, driven by the prospect of MOF-based devices. In this section the main methods used for MOF film preparation and MOF pattern fabrication will be illustrated, highlighting the most relevant progress and the conceptual differences.

Chemical solution growth. Solvothermal methods developed for MOF powder synthesis have been adopted for thin film growth. In some cases, nucleation and growth on the substrate occurs spontaneously, as in a particularly popular ZIF-8 growth protocol. ${ }^{177}$ In other cases, the surface needs to be pretreated, by the introduction of anchoring sites or organic ${ }^{178}$ or inorganic ${ }^{179,180}$ affinity layers. This approach is somewhat related to seeding protocols to locally lowering the energetic barrier for crystal nucleation. ${ }^{181}$ In any case, care should be taken to avoid corrosion and chemical contamination when introducing a substrate with previously defined features in a solution containing metal salts or at non-neutral pH levels. For this reason, mild conditions and minimally corrosive MOF precursors are preferred. Alternatively, the MOF could be deposited as film at the start of a fabrication sequence, before more delicate features are introduced. Top-down patterning of MOF films through lithography is not yet routinely performed, but has been illustrated for selected materials through photoresist masking and acid etching (Fig. 10a). ${ }^{108,182}$ This approach can likely be extended to other film growth methods and materials.

Bottom-up patterning, i.e. inducing patterned surface growth from a synthesis solution layered on top, has been demonstrated as well. For instance, carboxylate nucleation sites can be patterned on a surface while the rest of the surface is passivated with a $\mathbf{C F}_{3}$-terminated self-assembled monolayer (SAM). ${ }^{183}$ Similarly, seeds can be deposited in a pattern, e.g. by placing them in lithographically defined wells or channels. ${ }^{184,185}$ An alternative approach for direct lithographic control over nucleation is by including a photobase in the synthesis solution and irradiating a liquid film through a mask to locally generate basic species and induce MOF nucleation through ligand deprotonation. ${ }^{186}$ In evaporation-induced bottom-up patterning, MOF crystals are grown by depositing small volumes of precursor solution on predetermined locations on a substrate, followed by slow evaporation. ${ }^{187}$ The localization of the MOF precursor solution during evaporation gives rise to an increased precursor concentration in a confined volume, thus forcing nucleation and growth of a limited
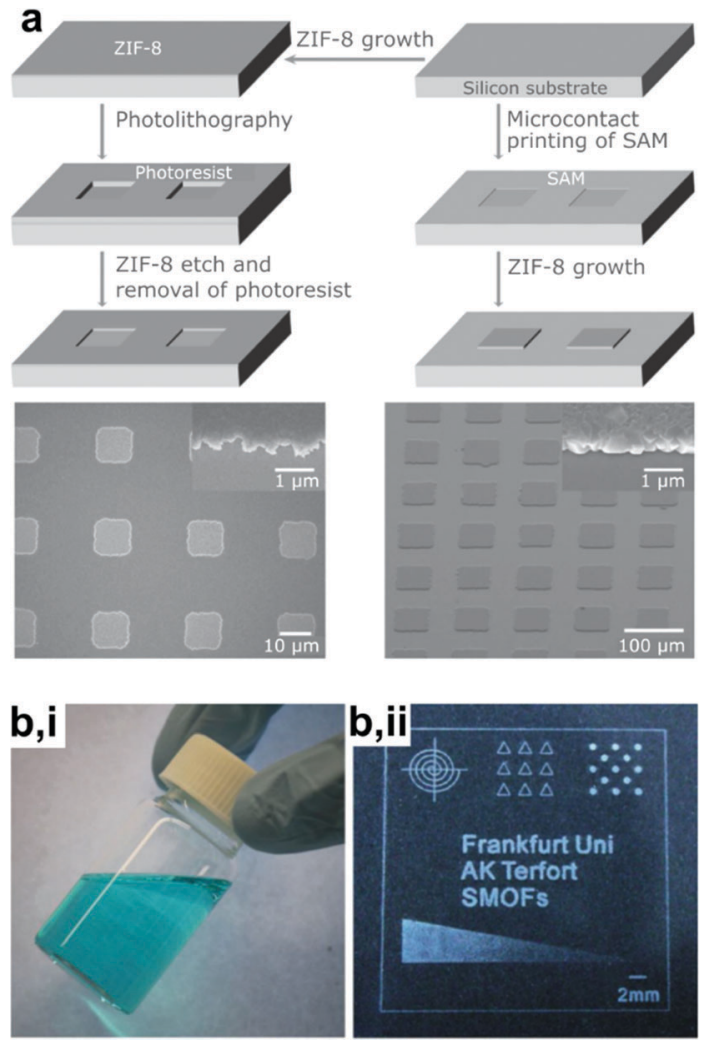

Fig. 10 Chemical solution growth of patterned MOF films. (a) Demonstration of two patterning routes for microscopic patterning of ZIF- 8 thin films via solution deposition: photolithographic etching (left) and areaselective solution deposition on microcontact printed SAM (right). Adapted from ref. 108. Copyright 2012 John Wiley \& Sons. (b) Inkjet printing of HKUST-1 precursor solution. (b,i) Picture of the precursor solution ink. (b,ii) Picture of the printed HKUST-1 pattern. Reproduced with permission from ref. 190. Copyright 2013 John Wiley and Sons.

number of crystals. This approach has been used to yield a few or even single crystals at each precursor droplet location. In several cases the orientation of these crystals with respect to the surface of the support was observed, likely due to the alignment of well-defined crystal facets with the surface. Different precursor solution deposition methods including micro-contact printing, ${ }^{187}$ microfluidic pen lithography, ${ }^{188}$ digital microfluidics, ${ }^{189}$ and inkjet printing ${ }^{190}$ (Fig. 10b) have been used to pattern MOF growth, even on large areas. For MOFs that can form under mild evaporation conditions, these methodologies provide a straightforward fabrication route.

Liquid-phase epitaxy. Liquid-phase epitaxial (LPE) deposition is a bottom-up layer-by-layer growth method that offers a high level of control over chemical functionalization and crystal orientation. Typically, SAM-functionalized gold-coated substrates are used with terminating functional groups that can interact with metal ions (e.g. $\left.-\mathrm{COOH},-\mathrm{NH}_{2},-\mathrm{OH}\right)$. By alternatingly exposing these substrates to metal salt and ligand solutions, a crystalline MOF film can be built up layer by layer. ${ }^{191}$ The resulting MOF films typically have a precisely controlled thickness and good properties in terms of homogeneity and roughness. These properties allow combination with other 
deposition techniques, as demonstrated by the fabrication of periodic MOF/oxide stacks via alternating LPE and sputtering. ${ }^{192}$ Additionally, crystals can often be grown with a clear out-ofplane preferential orientation and hetero-epitaxial MOF-on-MOF growth has been demonstrated. ${ }^{193-196}$ A recent review by Zhuang, Terfort and Wöll highlights different aspects of this deposition technique in detail. ${ }^{197}$ As LPE is greatly facilitated by using a metal precursor with a structural motif that resembles the MOF metal ion node, research in this area focused in particular on carboxylate MOFs based on $\mathrm{Cu}$ (II), $\mathrm{Zn}$ (II), and to a lesser extent $\mathrm{Fe}(\mathrm{III}){ }^{198}$ by using the corresponding acetate salts. ${ }^{193,194,199}$ Nevertheless, the demonstration of LPE growth of the azolate frameworks ZIF- ${ }^{200}$ and ZIF- $90^{201}$ indicates the broader applicability of the method.

LPE deposition was initially based on dip-coating, resulting in a time-consuming film fabrication process. A further iteration by Wöll, Fisher and co-workers demonstrated that alternatingly spraying the building block solutions preserves most of the characteristics of the original process and allows faster deposition on larger surfaces (Fig. 11a). ${ }^{202}$ Another advancement was made by Eddaoudi and co-workers, ${ }^{203}$ who demonstrated LPE deposition through alternating injection of MOF
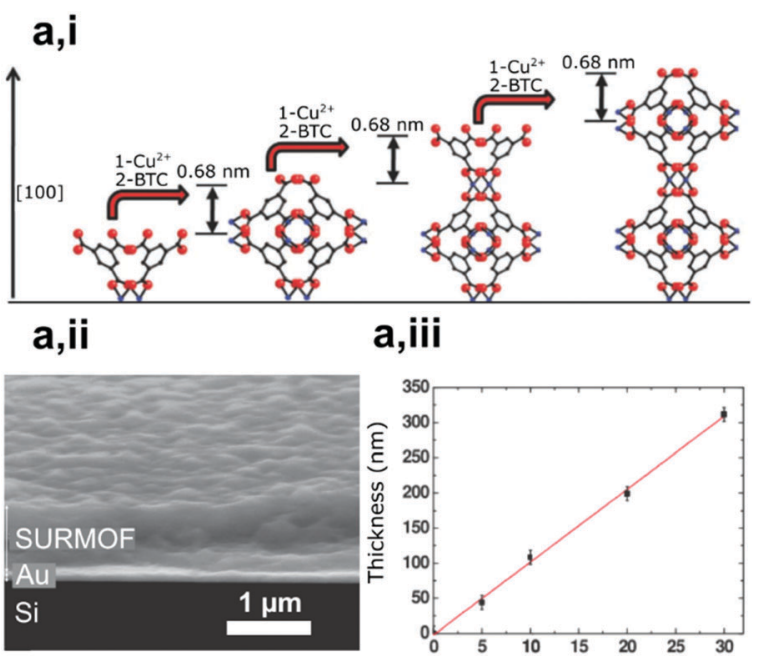

$a$,iii
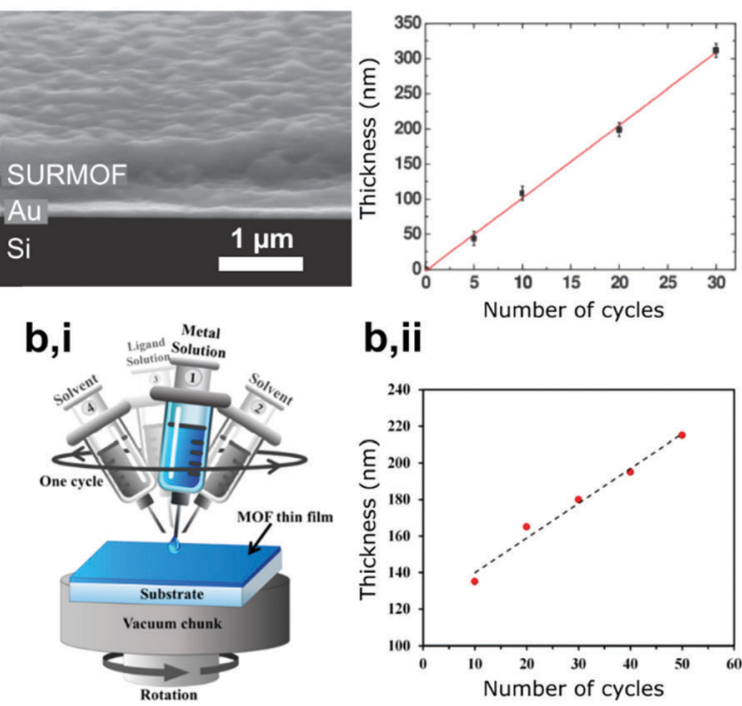

b,ii

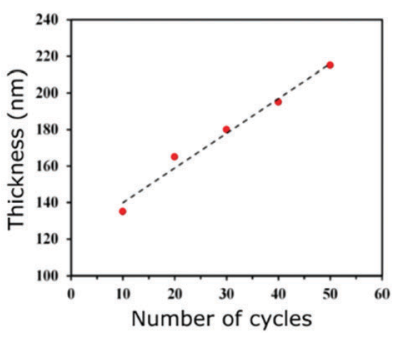

Fig. 11 Liquid phase epitaxy. (a,i) Schematic diagram showing LPE growth of $\{100\}$ oriented HKUST-1 SURMOF. (a,ii) Tilted-view electron microscopy image of spray LPE HKUST-1. (a,iii) Thickness of spray LPE HKUST-1 film at different number of cycles. Adapted from ref. 202. Copyright 2011 John Wiley \& Sons. (b,i) Overview of spin coating LPE method. (b,ii) Thickness of spin coating LPE HKUST-1 film at different number of cycles. Reproduced with permission from ref. 203. precursors in a spin-coater, thereby enabling rapid growth up to several micrometers for substrates up to tens of centimeters (Fig. 11b). In all accelerated LPE demonstrations, films were deposited on flat surfaces as draining issues would lead to inhomogeneity for complex three-dimensional substrates. The LPE methodology is intrinsically suitable for localized deposition, as the SAM surface functionalization required to initiate growth can easily be patterned, for instance via soft lithographic methods (e.g. stamping). ${ }^{204}$

Conversion of metal oxides and related precursors. The approach of using metal oxide films as precursors for MOF coatings benefits from a range of established technologies for deposition of the former (PVD, CVD, ALD, sol-gel methods, etc.). In the context of device fabrication, an advantage of this approach is that no corrosive metal salts are contacted with preformed circuitry (e.g. $\mathrm{Cl}^{-}$or $\mathrm{NO}_{3}{ }^{-}$anions). To grow the MOF, the oxide is decomposed to release cations while a suitable ligand is introduced. For example, the surface of an $\mathrm{Al}_{2} \mathrm{O}_{3}$ support was transformed into MIL-53 through solvothermal treatment in the presence of terephthalic acid. ${ }^{205}$ Similarly, Furukawa and co-workers illustrated the fabrication of MOF structures through quantitative pseudomorphic replication of three-dimensional $\mathrm{Al}_{2} \mathrm{O}_{3}$ structures fabricated via sol-gel routes. ${ }^{206}$ Recently, several reports demonstrated the conversion of thin ALD films instead of bulk oxides to conformal MOF coatings by solvothermal conversion, on both flat substrates and particles (Fig. 12a). ${ }^{207-209}$ As is clear from the thin film work, it is crucial to match precursor dissolution and MOF nucleation to avoid loss of a too large fraction of metal ions to the solution, resulting in defective coatings or even homogeneous (bulk) nucleation in the solution. Therefore, often fairly aggressive conditions not compatible with microelectronics fabrication are used (e.g. microwave heating, $\mathrm{pH}$ conditions). To completely avoid metal ions escaping to solution, the conversion can be performed under solvent-free conditions as well, as proposed by Stassen et al. for conversion of ZnO into ZIF-8 by contacting the oxide with the melted linker (Fig. 12b). ${ }^{210}$ In a next iteration, the same group demonstrated the transformation of conformal ALD metal oxides in a vapor-only process, coined "MOF-CVD”, that enables highly uniform ZIF-8 films as thin as $50 \mathrm{~nm} .^{211}$ By avoiding the use of solvents, metal salts and aggressive conditions, this process is highly compatible with vacuum technology and CMOS microfabrication. ${ }^{172}$

In addition to oxides, other inorganics have been used as well. For instance, $\mathrm{Cu}(\mathrm{OH})_{2}$ to HKUST-1 conversion was demonstrated as a route for efficient bulk production. ${ }^{212,213}$ Starting from crystalline $\mathrm{Cu}(\mathrm{OH})_{2}$ nanostructures Takahashi, Falcaro and co-workers, demonstrated the first heteroepitaxial inorganic-MOF growth process, resulting in both in-plane and out-of-plane orientation of Cu-based MOFs on a centimeter scale. ${ }^{214}$ This approach provides access to anisotropic properties of MOFs and devices based on oriented crystalline films, hinting at some similarities with semiconductor wafers. In situ oxidation of metallic substrates can be a source of metal ions as well. For instance, HKUST-1 films have been formed by exposing copper plates different oxidizing agents, including ammonium persulfate, 

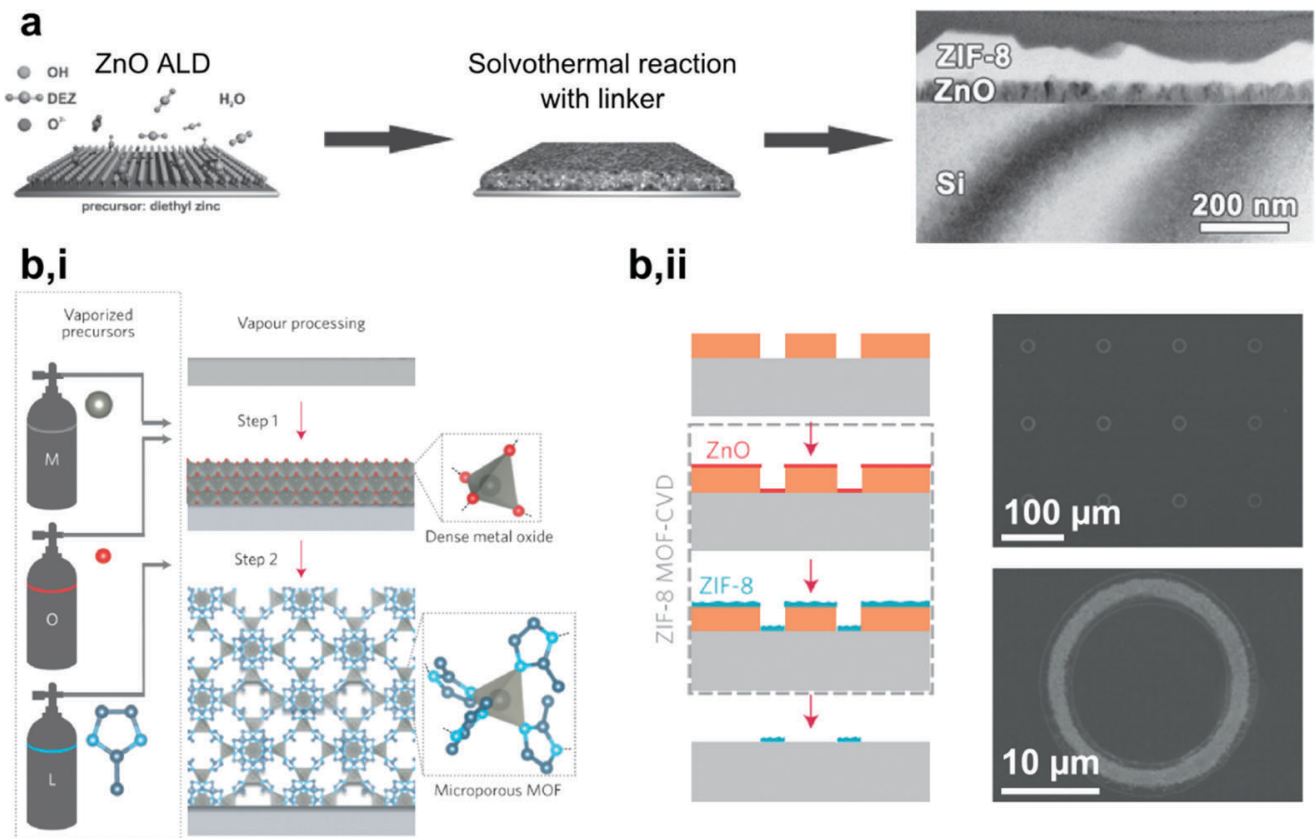

Fig. 12 Conversion of ALD deposited oxides. (a) ZnO deposition by ALD followed by partial solvothermal conversion to ZIF-8. Adapted from ref. 207. Copyright 2014 John Wiley \& Sons. (b,i) MOF-CVD route: ALD of ZnO followed by vapor-solid reaction with linker and crystallization of ZIF-8. $(b, i i)$ MOF-CVD lift-off lithography route for patterned deposition of ZIF-8. Adapted from ref. 210. Copyright 2016 Nature Publishing Group.

$\mathrm{H}_{2} \mathrm{O}_{2}$ and $\mathrm{Ag}^{+} \cdot{ }^{215}$ Grzybowski and co-workers extended this approach for different substrates (ceramics, metals and polymers) coated with metallic copper $(500 \mathrm{~nm})$ for the preparation of porphyrin-based MOF. ${ }^{216}$ Homogeneous MOF films were deposited over large areas $(>10 \mathrm{~cm})$ that could moreover be detached, allowing preparation of free-standing MOF membranes. As the conditions for direct metal conversion are intrinsically corrosive, this approach is more suitable for film deposition at the start of a fabrication process rather than later on when other features are present.

When thick films $(>0.5-1 \mu \mathrm{m})$ are targeted, the above methods are not particularly suited, at least not as a one-step approach. As the contact between ligand and inorganic is required, the process is limited by the diffusion of precursors through the MOF film. For most protocols, film roughness is somewhat higher than for LPE, up to $30 \%$ of the MOF film thickness. In terms of patterning, conversion of oxides and metals has the big advantage that it can rely on established protocols, often routinely applied in microfabrication. When solvothermally converting patterned precursors, particular care should be taken to match dissolution and MOF crystallization. As mentioned above, the immobility of metal ions in solventfree conditions has been demonstrated, thereby avoiding this issue. Solvent-free processing also enables entirely new patterning strategies, such as hot-pressing precursors to various substrates. ${ }^{217}$ In addition, vapor-only processing enables solvent-incompatible substrates and processing routes (e.g. lift-off patterning). Another advantage of growth from inorganics is that deliberate incomplete conversion leaves a "primer" layer between the MOF and the substrate, leading to improved adhesion.
Electrochemical film growth. Both anodic and cathodic electrochemical deposition processes have been reported for MOFs and were recently reviewed in detail. ${ }^{21,219}$ In anodic MOF formation, cations are liberated from a metallic electrode and react with ligand in the surrounding synthesis solution (Fig. 13a). This procedure was initially proposed by Müller and co-workers at BASF, ${ }^{20}$ as an method to avoid salts and therefore corrosion in the large-scale production of MOF powders. De Vos and co-workers realized the potential of this method for the rapid fabrication of HKUST-1 coatings due to the high concentration of $\mathrm{Cu}(\mathrm{II})$ ions at the anode. ${ }^{221}$ Several other teams further elucidated the underlying steps in film formation and the influence of experimental parameters such as solvents, temperature, electrolytes, voltage and current density on the growth of different MOFs including HKUST-1, ZIF-8, MIL-100(Al), MIL-53(Al), and MIL-53- $\mathrm{NH}_{2}(\mathrm{Al}) .{ }^{222-224}$ The method was later extended to films of a range of other well-known ZIFs (ZIF-4, ZIF-7, ZIF-14, ZIF-67) ${ }^{225}$ and robust Zr(Iv) frameworks such as UiO$66 .{ }^{226}$ Cathodic MOF deposition was first demonstrated by Dinca and co-workers and relies on the generation of basic species near the cathode surface (Fig. 13b). ${ }^{227}$ For instance, in a synthesis solution containing $\mathrm{Zn}$ (II) nitrate and terephthalic acid, the generation of $\mathrm{OH}^{-}$ions through reduction of the $\mathrm{NO}_{3}{ }^{-}$anion results in ligand deprotonation and formation of a MOF-5 film on the cathode. This approach was subsequently demonstrated for different probases and extended to other frameworks, including HKUST-1 and lanthanide-base materials. ${ }^{228-231}$ Although cathodic film growth requires the addition of a metal salt, it was also observed on the cathode during anodic UiO-66 growth in the presence of nitric acid, as $\mathrm{Zr}(\mathrm{Iv})$ species were transported from one electrode to the other. ${ }^{226}$ In this case, anodic deposition resulted 

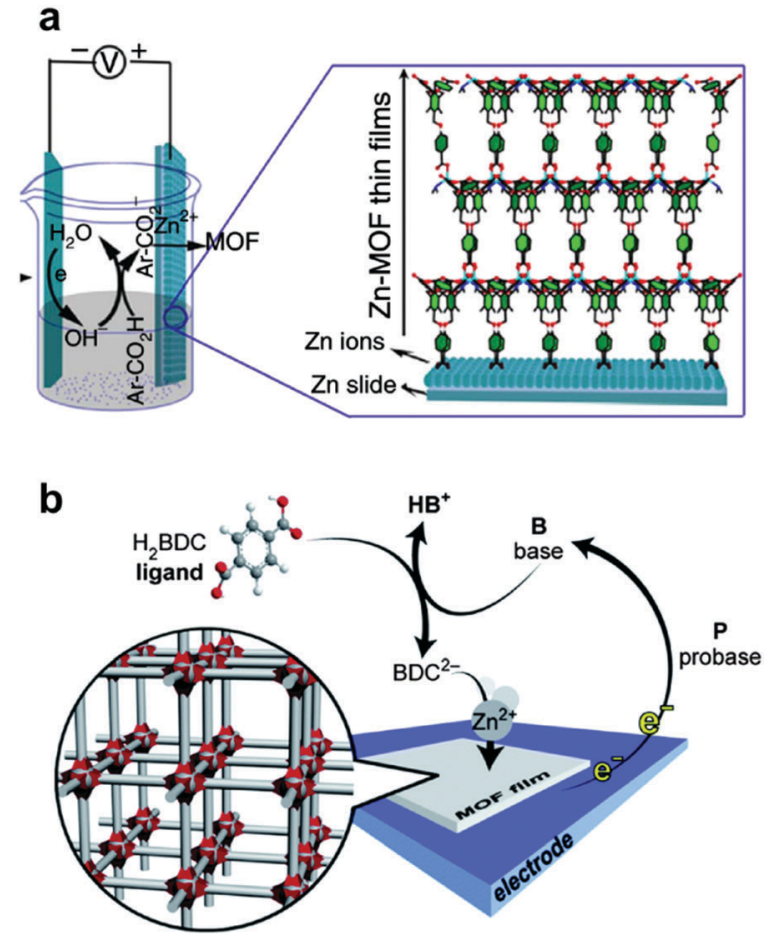

Fig. 13 Electrochemical film growth. (a) MOF deposition via anodic dissolution of metal ions. Reproduced with permission from ref. 165. (b) MOF deposition via cathodic reduction of a probase to stimulate linker deprotonation at the surface of the electrode. Reproduced with permission from ref. 229.

in superior adhesion on the metallic zirconium substrate, likely due to an intermediate oxide layer. On the other hand, cathodic deposition has the advantage that a broader range of electrodes can be used. For instance, reduction of a 4-carboxyphenyldiazonium salt was demonstrated to efficiently functionalize glassy carbon cathodes for subsequent solvothermal MOF deposition. ${ }^{232}$

For both anodic and cathodic approaches, the self-sealing character of film growth is an important advantage. The underlying mechanism is that when depositing non-conductive, electrode-passivating films such as MOFs, the uncovered metallic areas (i.e. film defects) provide a higher reaction rate, by releasing cations or producing basic species, and therefore enhance nucleation and growth. Mechanistic studies for the anodic case indicate that once a complete film is formed, metal ions can to some extent still pass through the MOF and initiate further growth at the MOF-solution interface, although care should be taken to avoid delamination. ${ }^{231}$ Another advantage of electrochemical deposition is the direct integration of the MOF films with an active electrode, thereby enabling straightforward electronic interfacing. ${ }^{165}$ Moreover, structuring electrochemical MOF growth is straightforward as nucleation and growth only occurs on the exposed metallic surface of patterned electrodes. $^{221,233,234}$ However, a requirement for controlled deposition is maintaining electrical contact. While trivial in the cathodic case, this condition hampers full metal conversion for the anodic process. An alternative wireless approach is bipolar electrodeposition, in which the metallic surface to be coated is placed in a synthesis solution in an electric field. If the polarization is strong enough, cathodic and anodic redox reactions can occur on opposite sides of the metal object, as demonstrated for ZIF-8 and HKUST-1 growth. ${ }^{235}$

Films of preformed MOF particles. As an alternative to direct synthesis on the substrate, preformed MOFs crystals can be transferred, either as a colloidal suspension or in the form of a powder. This methodology was first demonstrated by dip coating a MOF nanocrystal suspension to form homogeneous films in the $40 \mathrm{~nm}-1 \mu \mathrm{m}$ range. ${ }^{236,237}$ Spin-coating of colloidal suspensions has been used as well. ${ }^{238}$ Recently, a cold supersonic spray-coating method was demonstrated, where MOF particles are ejected with high kinetic energy (equivalent velocity of $\sim 500 \mathrm{~m} \mathrm{~s}^{-1}$ ) onto different substrates. ${ }^{239}$ Large-area deposition was shown in the range of tens of centimeters. This method most clearly offers opportunities for the deposition of relatively thick films (e.g. $20 \mu \mathrm{m}$ ZIF-8 films).

The solvent-air interface or the boundary layer between immiscible solvents can be used to grow a free-standing MOF film that can be subsequently transferred to a substrate. The Langmuir-Blodgett methodology, often used to assemble surfactant monolayers, was demonstrated for the deposition of two-dimensional coordinative network films and threedimensional frameworks. A review describing the key aspects of this technique such as preferred orientation was published by Kitagawa and co-workers. ${ }^{240}$ A similar method was employed to transfer films of the hydrophobic $\mathrm{Ni}_{2} \mathrm{HITP}_{3}$ material formed at the air-water interface to fabricate proof-of-concept transistors. ${ }^{28}$ In a related approach first demonstrated for HKUST-1 and ZIF-8, the metal salt and ligand are contacted as separate solutions in immiscible solvents. ${ }^{241}$ This approach was as well used to fabricate demonstrator transistors by transferring the film formed at the liquid-liquid interphase. ${ }^{46}$

Electrophoretic deposition of preformed MOF particles was first demonstrated by Hwang et al. to coat ZIF-69 on a microresonator sensor. ${ }^{242}$ This broadly applicable approach was subsequently expanded for HKUST-1, MIL-53, UiO-66 and NU-1000 by Farha, Hupp and co-workers. ${ }^{243}$ Interestingly, all of the examined MOFs display net negative charges and were deposited on the positive electrode. To tackle the often weak adhesion of larger physically deposited particles, Falcaro, Furukawa and co-workers used a glue-like primer to fix the MOF crystallites. $^{244}$ For example, by heating a commercial resist coating (SU-8) and pressing the softened polymer against a MOF powder bed, different materials could be efficiently immobilized by partial encapsulation. Similarly, phenyl-silane hybrid primers were prepared using the sol-gel method and doctor-bladed films of this material were used to bind ZIF-9 after solvent evaporation. ${ }^{245}$ The roughness, homogeneity and thickness of the final films strongly depend on the MOF particles. Additionally, control over the crystal orientation is unlikely. However, there are no limitations on the type of MOF that can be glued on a surface under mild heating $\left(<200{ }^{\circ} \mathrm{C}\right)$. Patterned deposition has been demonstrated as well for both primer approaches, by using pre-patterned photoresist features and X-ray lithography of the phenyl-silane material, respectively. 
In the latter case, deep X-ray lithography was used to circumvent scattering by the MOF particles and to induce cross-linking of the hybrid film with sufficient penetration depth. ${ }^{246}$

\subsection{Other fabrication uses of MOFs}

Templating is a rapidly growing aspect of MOF science. We review other types of templating, shown schematically in Fig. 14. The most straightforward approach, infiltration-reaction, infiltrates a preformed MOF with chemical precursors that are subsequently reacted to create nanoscale structures such as quantum dots or carbon nanotubes (Fig. 14a). Alternatively, a pre-synthesized nanostructure can be encapsulated by assembling the MOF around it (Fig. 14b). Hierarchical templating methods create composite materials with features that span beyond the local MOF pore network by using MOF crystals as a growth template ("deposition"; Fig. 14c).

Infiltration and reaction. The tailorable pores and welldefined structures of MOFs provide a versatile platform for directing the templated growth of ordered nanostructures within their pore space. The first step of this process involves the introduction of precursors into the pores via the gas, ${ }^{247-250}$ liquid, ${ }^{251-253}$ or solid phase,$^{254-256}$ followed by conversion (Fig. 14a). Hard template materials, such as zeolites and mesoporous silica, have been used as well in this way, but a key advantage of MOFs is their flexible synthetic chemistry that can be exploited to direct the nanostructure growth. For example, it has been demonstrated that the MOF structure and geometry can be used to control the size and orientation of nanoparticles or nanowires over a range inaccessible via traditional templating. ${ }^{257,258}$ Ordered arrays of silver nanostructures with diameters $<10 \mathrm{~nm}$

\section{$\mathbf{a}$}

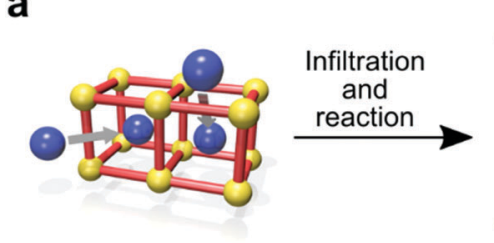

b

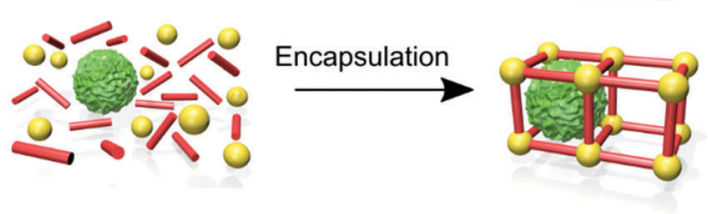

C
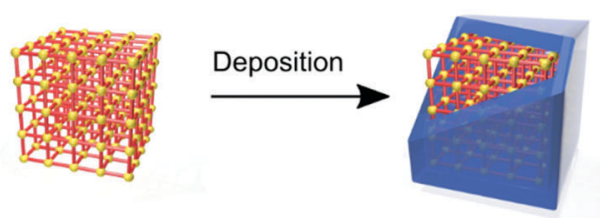

Fig. 14 Schematic overview of templating methods involving MOFs. (a) Precursor infiltration and subsequent nanostructure formation within MOF pores. (b) Nanostructure encapsulation during MOF synthesis. (c) Composite material synthesis using MOF crystals as templates. were formed by varying the silver salt loading within various MOFs before decomposition. ${ }^{257}$ A study building on this work showed the possibility of growing gold and platinum nanowires within the onedimensional MOF pores. ${ }^{259}$ The formation of metallic nanostructures is currently the most extensively investigated use of templatedirected reaction and has been demonstrated for a wide array of metals and alloys (e.g. Cu, Pd, Pt, Ru). ${ }^{260-262}$ These examples represent a first step toward the tantalizing goal identified in the first roadmap, namely to exploit MOF templating to arrange precisely spaced and oriented nanowires as electrical interconnects with nanometer-scale resolution. Nevertheless, major hurdles remain, not the least of which is the formation of electrical contacts between these nanowires and various device components.

For electronic devices, nanoscale particles can serve as quantum dots (QDs) that provide broad visible light absorption. By modifying their size the QD emission can be tuned to overlap with the lowest-energy absorption band of the MOF to allow efficient energy transfer for photocatalysis and lightharvesting. Fischer and co-workers provided a proof of concept by templating semiconducting $\mathrm{TiO}_{2}$ nanoparticles in MOF-5 through vapor phase titanium-isopropoxide infiltration. ${ }^{263}$ Since then, other QD@MOF materials have been created, including $\mathrm{ZnO}$ and GaN using ZIF-8 as a template and diethyl zinc $^{264}$ or trimethylamine gallane ${ }^{265}$ precursors. More recently, CdSe nanoparticles were incorporated into the pores of MIL$101(\mathrm{Al})-\mathrm{NH}_{2}$ via a similar templated infiltration reaction. ${ }^{266}$ As already mentioned in Section 2.3, perovskite nanodomains were formed inside the pores of MOF-525 when added to a perovskite precursor solution, resulting in an increase in solar cell efficiency that seems related only to the MOF scaffolding. ${ }^{116}$ Porphyrin-based MOFs are particularly attractive hosts for QD confinement for photonic devices because of their promising light harvesting properties (see Section 2.3) that could be further enhanced by confined QDs. Although composites of two Zn-porphyrin-based MOFs and amine-capped CdSe/ZnS core-shell QDs have been reported, ${ }^{267}$ the synthesis was achieved not via templating but via surface functionalization allowing only a single QD layer on the MOF surface. The improvement in light-harvesting efficiency was nevertheless substantial; $\sim 50 \%$ more photons could be absorbed over a monolayer of porphyrin building blocks in the MOF. Further efficiency improvements can be anticipated by incorporating well-dispersed QD in bulk porphyrin-based MOFs. The advantage of templating approaches is that they eliminate the need for stabilizing ligands or surfactants on the QD surface, as typically used in template-free solution synthesis. Moreover, they enable the formation of ordered QD arrays interesting for imaging and display applications.

Another promising class of MOF-based templating reactions involves the polymerization of monomer precursors to achieve polymers with controlled molecular weight and stereochemistry guided by the confinement in the MOF pores. ${ }^{268}$ Recent studies have expanded the repertoire of conductive polymers that can be formed using MOFs, now including poly-3,4-ethylenedioxythiophene (PEDOT) ${ }^{269}$ polythiophene, ${ }^{270}$ polyaniline ${ }^{271}$ polymethylpropylsilane, ${ }^{272}$ poly $(N$-vinylcarbazole $),{ }^{273}$ polyacetylene, ${ }^{274}$ 
and polypyrrole. ${ }^{275}$ Demonstrations of the potential benefits of this synthetic strategy in electronic devices are also now emerging. In particular, recent work shows that MOF-polymer guest@MOF composites not only exhibit enhanced electronic conductivity relative to the bulk polymer or evacuated framework but that these properties persist once the MOF template is removed. $^{269,272,273}$ The origin of these property enhancements is rooted in the conformation control the MOF provides. For example, the enhanced charge carrier mobility that results from confining single polysilane chains within DUT- 4 and DUT- 5 is caused by the elimination of slow inter-chain hole hopping that is otherwise present in bulk polysilane. ${ }^{272}$ As an added benefit, the host can also enhance the chemical stability of the polymer, as illustrated by the improved photostability of MOF-confined polysilane.

Encapsulation during MOF synthesis. The second nanoscale templating category entails growing the MOF around a preexisting template. One advantage of this approach over the infiltration-reaction route is that it is a simple one-pot procedure. Moreover, the MOF is not required to be stable under the reaction conditions used to form the template, which resulted in encapsulating a wider range of QD materials: ${ }^{276} \mathrm{CdTe},{ }^{277}$ graphene, ${ }^{278}$ and carbon nanocrystals ${ }^{279}$ in $\mathrm{ZIF}-8, \mathrm{TiO}_{2}$ in HKUST- $1,{ }^{280} \mathrm{CdSe}$ in a Eu(III) MOF, ${ }^{281}$ and $\mathrm{CdSe} / \mathrm{CdS} / \mathrm{Cd}_{0.5} \mathrm{Zn}_{0.5} \mathrm{~S} /$ ZnS multishell QDs in MOF-5. ${ }^{282}$ Among these studies, the resulting composite has mostly been tested for sensing applications, ${ }^{276}$ leaving improved energy harvesting in QD@MOF materials still largely unexplored. In that context, the stabilization by encapsulation of porphyrins and metalloporphyrins is of interest as well; details of these efforts are summarized elsewhere. ${ }^{283,284}$ Because of their relatively complex size and shape, porphyrins can also play a structure-directing role that enables the templated formation of MOF structures otherwise unattainable. ${ }^{284}$

Material synthesis using MOF templates. MOFs can serve as sacrificial templates that are removed once the desired microstructure is formed. In a recent demonstration of this concept, Telfer et al. showed that ZIF-8 and ZIF-67 crystals can serve as templates for creating hollow titania shells, porous titania, and titania-based composite nanomaterials. ${ }^{285}$ In addition to enabling tailored nanoparticle morphology and composition control, the authors demonstrated that components of the MOF templates, including encapsulated guest molecules, could be incorporated within a nanoparticle shell following the decomposition of the MOF. One particularly promising use of MOF templating is the synthesis of porous carbons with electrochemical storage characteristics interesting for batteries and supercapacitors. The first demonstrations of this concept were based on the carbonization of MOF-5 after infiltration with various precursors, resulting in high surface-area carbons with high specific capacitance and suitable galvanostatic charge-discharge curves. ${ }^{286-288}$ Recent work shows that through the carbonization and subsequent potassium hydroxide activation of ZIF-8 nanoparticles yields hierarchically porous carbons with unprecedentedly high supercapacitive properties. ${ }^{289}$ Moreover, the nitrogen-doped carbons produced from ZIF pyrolysis have potential as fuel cell catalysts. ${ }^{290,291}$ To produce carbons, the metal ions of the MOF framework must be removed after or during pyrolysis. Another approach is to view the MOF itself as a nanoscale organic-inorganic composite. By pyrolysis of a suitable transition metal MOF (e.g. ZIF-67), it is therefore possible to generate a porous carbon matrix with embedded metal oxide particles (e.g. $\left.\mathrm{Co}_{3} \mathrm{O}_{4}\right)$ with the same composition as used in batteries. In this scenario, the carbon matrix simultaneously provides electrical contact, prevents sintering of the active phase and buffers mechanical stresses, e.g. during intercalation-deintercalation in Li-ion batteries. For an in-depth discussion of work in this area, we refer to recent reviews. ${ }^{292,293}$

\subsection{Mechanical properties of MOF thin films}

The measurement, understanding and alteration of mechanical properties of functional materials is of critical importance in the context of miniaturized devices as mechanical loading (e.g. bending, torsion, compression) is practically unavoidable, during microfabrication, back-end processing or real-world operation. Mechanical loading can induce structural damage, which for MOFs may show as phase changes, (partial) pore collapse and eventually amorphization. Insight in the behavior of MOFs under mechanical stress has grown considerably over the last decade, through combined computational and experimental studies, mostly based on single crystals or microcrystalline powders. Mechanical properties such as the shear modulus, bulk modulus, elastic modulus, Poisson's ratio and hardness have been determined for a few MOFs through nanoindentation, high-pressure crystallography and molecular dynamics simulations. ${ }^{294}$ In general, it has been found that MOFs exhibit "softer", i.e. more flexible and less hard, properties in comparison to inorganic materials such as zeolites and microporous oxides. On material selection maps, MOFs can therefore be roughly placed in an intermediate position between inorganics and polymers (Fig. 15). Intuitively, this is

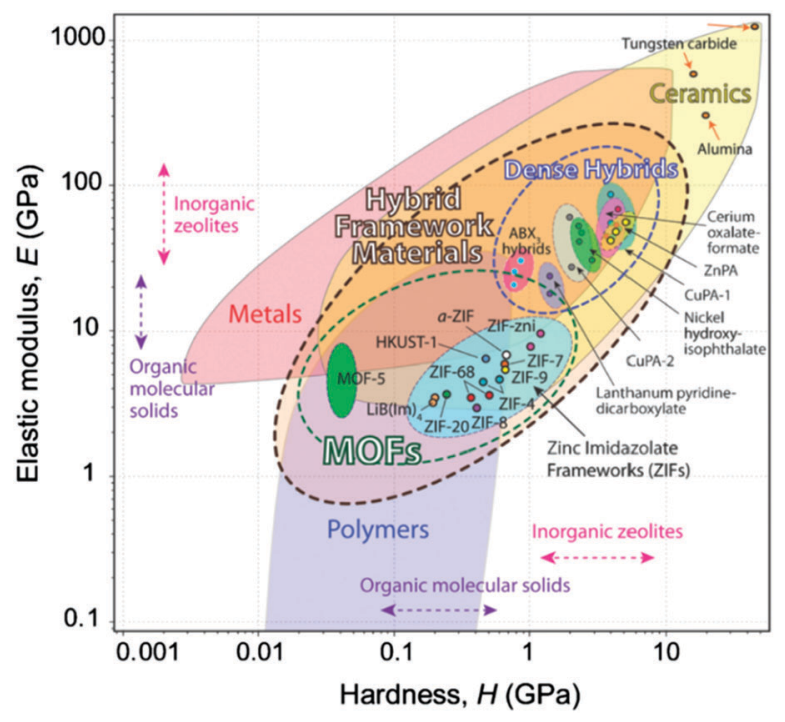

Fig. 15 Elastic modulus vs. hardness material property map. The measured mechanical properties of some typical MOFs are found in an intermediate position between classical groups of hard and soft materials (metals, polymers, ceramics). Reproduced with permission from ref. 294. 
explained by their larger porosity, more bendable organic building blocks and lower bond strengths. However, MOFs often exhibit "anomalous" mechanical behavior related to their structural flexibility and the diverse chemical interactions that can take place within the pores. ${ }^{295}$ For instance, anisotropy of bond strengths and framework rigidity causes different mechanical properties for different crystal faces. ${ }^{296}$ Extreme anisotropy of the compressibility of MOFs has also been observed and related to similar mechanisms. ${ }^{297,298}$ Strain-induced phase transformations can lead to rather extreme (reversible) compression of the unit cell volume. ${ }^{299,300}$ In addition, the stimuli-responsiveness of MOFs has been a celebrated topic of research, ${ }^{301,302}$ including adsorptioninduced reversible structural transitions such as breathing, ${ }^{303}$ gateopening, ${ }^{304}$ multistep adsorption, ${ }^{305}$ and recently the phenomenon of guest-induced contraction. ${ }^{306}$ Giving a complete overview of such behavior is beyond the scope of this review; we refer to a recent perspective for further reading. ${ }^{302}$

The occurrence of lattice defects (e.g. in the form of missing linkers or nodes) causes structural destabilization and might explain discrepancies between computationally predicted properties of "ideal" frameworks and observed experimental results. ${ }^{307}$ On the other hand, such defects can be used as anchoring sites for chemically tuning the mechanical properties. $^{308}$ In thin films, factors such as anisotropy, texture or grain boundaries may cause additional deviations from the predicted properties. For example, Wöll and co-workers observed an elastic modulus $(9.3 \mathrm{GPa})$ and hardness $(0.23$ GPa) for $\{100\}$ oriented LPE HKUST-1 thin films, ${ }^{309}$ which closely matched with computed values. By contrast, randomly oriented electrochemically grown films of the same material displayed significantly lower elastic modulus (3.5 GPa) and hardness $(0.17 \mathrm{GPa}){ }^{310}$ Nevertheless, a close match between the mechanical properties of films and single crystals can be considered an indication of film quality in terms of phase purity, absence of interstitial gaps and cohesion of the crystallites. For example, Eslava et al. determined an elastic modulus (3.5 GPa) and hardness $(0.43 \mathrm{GPa})$ for polycrystalline ZIF-8 films ${ }^{160}$ that were close to those previously determined for the $\{110\}$ face of a ZIF-8 single crystal (2.97 GPa and $0.50 \mathrm{GPa}$, respectively). ${ }^{311}$

The integration of MOF thin films in devices implies interfacing with different materials, for instance within vertical stacks. As guest-induced flexibility phenomena typically involve large lattice parameter changes, it will be interesting to see how and to what extent this behavior is influenced by interfacing with a substrate. In one of the few studies in this context, Kitagawa and co-workers have shown through grazingincidence X-ray diffraction on single crystals that a guestinduced sheared phase of $\mathrm{Zn}_{2}(\mathrm{NDC})_{2}(\mathrm{DABCO})$ at the surface can remain connected to the unaltered lattice in the bulk. ${ }^{312}$ Perhaps even more important, dissimilar material properties in vertical stacks will cause (interfacial) mechanical strain especially when exposed to stimuli (e.g. guest molecules, temperature changes or pressure swings), potentially resulting in failure through debonding or cracking. For instance, the brittleness of MOF film relative to ductile polymer substrates can lead to additional microcrack channeling and film debonding

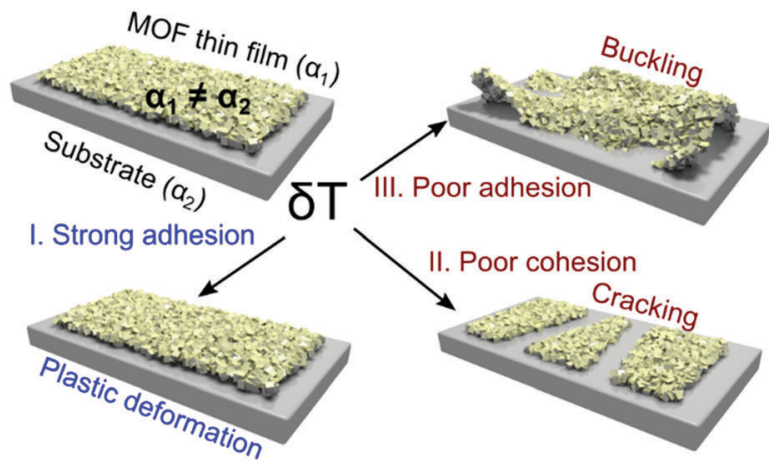

Fig. 16 Effects of residual stress on film properties due to a mismatch in the thermal expansion coefficient, $\alpha$, of the film and its substrate caused by variations in temperature during MOF film processing and device operation.

failure modes, e.g. induced through tensile strain upon substrate bending. ${ }^{313}$ To assess the viability of integration of MOF thin films with flexible electronics, bending testing of such assemblies should be conducted. Two rare example will be discussed in Section 4.1: HKUST-1/PET and ZIF-8/PET (PET = polyethylene terephthalate) stacks for which the performance as memory resistors was tested at different bending radii and associated levels of tensile strain. ${ }^{314,315}$ As a second example, a mismatch in the coefficient of thermal expansion (CTE) between a thin film and its substrate can produce residual stresses following growth that can cause material fatigue or even compromise the connection between the MOF and its electrical contact (Fig. 16). ${ }^{316}$ Consequently, it is important to understand this behavior so that device reliability issues can be avoided by modifying or minimizing temperature changes during film growth and processing and device operation. The CTE of MOFs can be quantified experimentally through refinement of the lattice parameters during variable temperature X-ray or neutron diffraction measurements. Despite the relatively large number of reported variable-temperature powder X-ray diffraction experiments, only a few MOF CTE values have been determined. In most cases, these concern MOFs that exhibit negative thermal expansion, which is a relatively rare property that nevertheless has been predicted to be widespread in nanoporous materials. ${ }^{317}$ For example, HKUST- $1^{318}$ and MOF- $5^{319}$ have CTE values near room temperature of roughly $\alpha=\mathrm{d} L L^{-1} \mathrm{~d} T^{-1}=-4.1 \times 10^{-6} \mathrm{~K}^{-1}$ and $-1.4 \times 10^{-5} \mathrm{~K}^{-1}$ ( $L=$ length), respectively. A limited number of other MOFs also show negative thermal expansion. ${ }^{320-324}$ In contrast, the CTE of the substrates commonly used in electronic devices tend to be positive near room temperature. Reported values $\left(\mathrm{K}^{-1}\right)$ for silicon, copper, aluminum, and silver are $\alpha=2.6 \times 10^{-6}, 1.7 \times 10^{-5}, 2.3 \times 10^{-5}$, and $1.9 \times 10^{-5}$, respectively. ${ }^{325,326}$ This large CTE mismatch between a MOF thin film and its substrate could lead to film cracking or delamination. Negative thermal expansion is of interest for many composite material applications, however. ${ }^{327}$

The thermal expansion properties of MOFs can also be predicted using simulation techniques such as NPT (constant number of particles, pressure, and temperature) molecular 
dynamics. ${ }^{328-331}$ This approach was used to yield CTE values in reasonable agreement with those measured for MOF-5 and also predicted the increase in negative thermal expansion with increasing linker length. ${ }^{329-331}$ Performing these thermal expansion calculations requires accurate flexible force field models of MOFs; unfortunately, the limited number of such models developed for MOFs makes it difficult to predict CTE trends on a larger scale. Although CTE can also be predicted using DFT, the timescale required for thermal equilibration of the lattice parameters can make these calculations prohibitively expensive. However, quantum mechanical calculations can still play a valuable role in elucidating the underlying vibrational modes that govern the thermal expansion properties of the framework. ${ }^{319}$ The potentially large range of CTE values possible in MOFs could also have important implications for their use in applications where fluctuations in temperature can occur during device operation. For example, in devices that rely upon a mechanical transduction mechanism for sensing applications such as microcantilevers, variations in temperature can create stresses that modify the sensing response. ${ }^{332}$ This further motivates the need for thermal expansion measurements to be performed on additional MOFs in the literature. An additional challenge exists for MOFs that exhibit discontinuous changes in their CTE due to temperatureinduced phase transitions. Such behavior is observed in MIL-53(Al), for which a large anisotropic change in lattice parameters occurs during the temperature-induced phase transition between its large-pore and narrow-pore conformations. ${ }^{333,334}$

\section{Case studies of MOFs in electronic devices}

In this section we highlight recent progress on integrating MOFs in electronic devices. We refer to the definition of "electronic devices" in the Introduction, as hardware that uses current or voltage as input or output and in which a MOF enables a function such as sensing, energy conversion, data storage or logic processing. Following publication of the first roadmap in 2011, several milestones in MOF-based devices have been realized, as well as important progress in this direction. MOF-enabled sensing continues to be a particularly active area of research and has been thoroughly reviewed, ${ }^{335}$ most recently in $2014 .^{93}$ In contrast to previous overviews of this area, recent progress allows us to adhere more strictly to the definition of an electronic device, which shifts the focus from chemistry- to more engineering-focused. For instance, although there are many reports on sensing-relevant properties of MOFs, such as changes in color or luminescence upon uptake of chemical species, we do not consider these devices. We make this distinction to emphasize that the integration of MOFs in electronic and photonic applications must go beyond materials discovery to include processing and material integration technologies. Although not exhaustive, the case studies we picked serve to highlight the diversity of roles MOFs can play in electronic devices, a versatility that stems directly from the range of properties discussed in Section 2.

\subsection{Digital circuits}

Digital (electronic) circuits are broad group of devices for processing and storage of logic signals (signal/ground voltage = ON/OFF). Since miniaturization of integrated digital circuits is one of the key pillars of our current information technology and further downsizing of current CMOS technology will come to an end in the foreseeable future, new approaches and novel materials are explored with increased interest. New trends such as lowpower/low-cost standalone devices (e.g. "internet-of-things" applications) and flexible electronics are further inciting this interest. The work on graphene-based devices is a prominent example of this development and is recently expanding to compositionally more versatile and nanoporous analogues. ${ }^{336}$ We demonstrate the potential of MOF integration in this context through a discussion of the progress in two categories of devices: logic gates and memory cells. Potential distinguishing features of MOFs in these applications are the (sub-)nanometer ordering, the tunable electronic band structure and the intrinsic open pore space that can be exploited for guest@MOF approaches.

4.1.1 Field effect transistors. Field effect transistors (FETs) are three-terminal circuit elements operated as logic gates, i.e. electronic switches. A FET consists of a semiconductor, a dielectric and three conducting electrodes (gate, source and drain). The device can be viewed as a planar capacitor, formed by the gate electrode and semiconductor on both sides of a dielectric (Fig. 17). Applying a voltage to the gate electrode $\left(V_{\mathrm{G}}\right)$, i.e. charging the capacitor, causes charge carrier depletion or enhancement in the semiconductor and thereby tunes the current flowing between the drain and source terminals $\left(I_{\mathrm{DS}}\right)$. In practical digital circuits, the device is optimized for ON/OFF switching based on the logic input on the gate terminal. In addition, FETs are also highly useful experimental tools for indepth characterization of semiconductors. The key parameters to compare device performance are the charge carrier mobility and the threshold voltage, as described elsewhere. ${ }^{163}$

A challenge in fabricating FETs to study crystalline materials stems from the fact that charge transport occurs only in the first few molecular layers of the semiconductor. Electronic percolation within these layers is required for device operation. Importantly, for polycrystalline MOF films, several effects of the particle-particle and semiconductor-dielectric interfaces, such

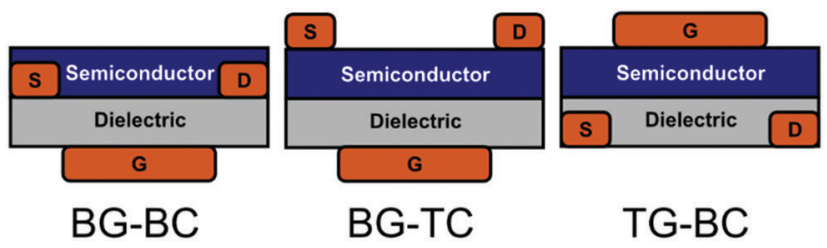

Fig. 17 Three geometries that are widespread for FET integration and testing of organic, and recently hybrid, semiconductors. From left to right: bottom gate, bottom contacts $(B G-B C)$, bottom gate, top contacts (BG-TC), top gate, bottom contacts (TG-BC). Redrawn from ref. 163. 
as grain boundaries, interfacial adhesion and roughness, affect contact quality and charge carrier transport and are therefore expected to interfere with the interpretation of the device performance. ${ }^{337}$ It is noteworthy that single crystal MOF FETs in which such effects would be minimized are still to be realized.

Panda and Banerjee reported the characterization of a twodimensional In(III)isophthalate framework using a FET. ${ }^{129}$ In this work, BG-BC FETs were fabricated by drop casting the crystals on pre-fabricated device structures. From the $I_{\mathrm{DS}}-V_{\mathrm{G}}$ transfer curve of the device, p-type depletion behavior and a typical threshold voltage and carrier mobility of $-2.0 \mathrm{~V}$, respectively $4.6 \times 10^{-3} \mathrm{~cm}^{2} \mathrm{~V}^{-1} \mathrm{~s}^{-1}$ was found. The charge mobility was linked to close $\pi-\pi$ stacking of the two-dimensional layers in the framework. Zhu and co-workers reported the characterization of a two-dimensional Cu-BHT (BHT = benzenehexathiol) coordination polymer using a FET. ${ }^{46}$ In this work, the material was directly synthesized as a free-standing polycrystalline thin film at the interface between immiscible aqueous and organic precursor solutions. The thin films were transferred to a prefabricated device structure to form BG-BC FETs. From the transfer characteristics of the device, high ambipolar transport was found, with up to $99 \mathrm{~cm}^{2} \mathrm{~V}^{-1} \mathrm{~s}^{-1}$ and $116 \mathrm{~cm}^{2} \mathrm{~V}^{-1} \mathrm{~s}^{-1}$ hole and electron mobility, respectively. This behavior is typical for a FET fabricated from a gapless conductor. However, the observed mobility surpasses all previously reported organic ambipolar devices. Therefore, further research on the manipulation of the electronic band structure, for example through doping, could lead to very promising FET devices based on similar coordination polymers.

Although not strictly porous, the results for the above materials are relevant for the further development of MOFs in this area. $\mathrm{Xu}$ and co-workers recently reported the first FET that implemented a porous MOG, namely $\mathrm{Ni}_{3}(\mathrm{HITP})_{2}$, as active channel (Fig. 18). ${ }^{28}$ Physisorption measurements confirmed a pore volume and BET surface area of $0.51 \mathrm{~cm}^{3} \mathrm{~g}^{-1}$ and $625 \mathrm{~m}^{2} \mathrm{~g}^{-1}$, respectively. Polycrystalline free-standing thin films were formed at the air-water interface of a $\mathrm{Ni}_{3}(\mathrm{HITP})_{2}$ synthesis solution. Next, BG-TC devices were fabricated through transferring the films to a $\mathrm{SiO}_{2} / \mathrm{Si}$ substrate, followed by gold contact evaporation through a shadow mask. The top surface of the interfacial film was very smooth (RMS roughness of $\sim 1 \mathrm{~nm}$ ), which is thought to be responsible for the low estimated defect density in the resulting devices. From the transfer characteristics of five devices, p-type depletion behavior and a typical threshold voltage of $1.1 \mathrm{~V}$ and average carrier mobility of $38 \pm 8 \mathrm{~cm}^{2} \mathrm{~V}^{-1} \mathrm{~s}^{-1}$ were found. The device moreover displayed on/off current ratios as high as 2000 . The observed mobility is competitive with state-of-the-art solution-processed organic and inorganic semiconductors, a remarkable feature for a material that consists for a significant part of empty pore space. The authors pointed out that no saturation current was observed and that the device showed gapless conductor behavior, similar to the $\mathrm{Cu}-\mathrm{BHT}$ case.

4.1.2 Resistive memories. Memory resistors, or in short "memristors", are two-terminal circuit elements that display bipolar switching of resistance through field-dependent hysteresis. Current-voltage behavior of the element can be switched between a high and low resistance state by opposite writing and erasing voltage sweeps, i.e. storing digital ON and OFF signals. Importantly, in order to be interesting for low-power applications, switching needs to occur at relatively low threshold voltage. Switching in memory resistors can either be based on physical processes, such as phase transitions, charge trapping, ferroelectrics or magnetics, or on chemical processes, typically involving the reversible formation of mobile ion gradients or passivation layers. ${ }^{338}$ Although relatively new circuit elements, memristors based on ceramics are promising for integration in
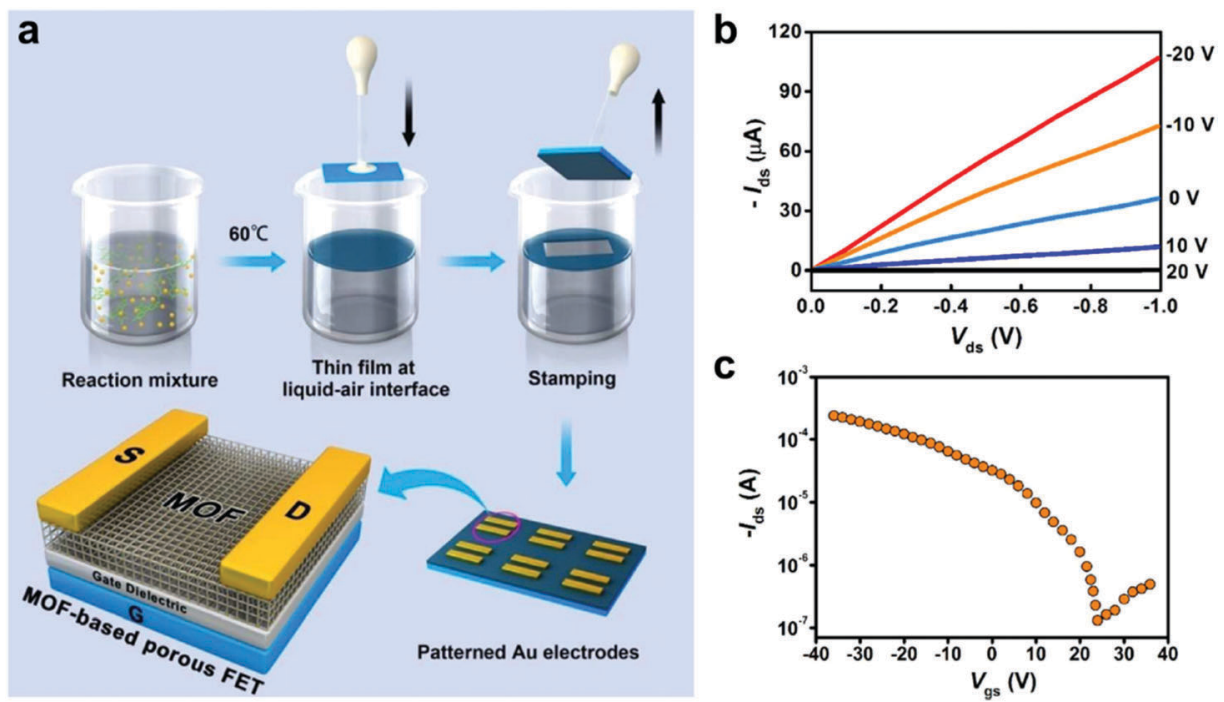

Fig. 18 Proof-of-concept demonstration of $\mathrm{Ni}_{3}(\mathrm{HITP})_{2}$ field effect transistor. (a) Schematic representation of fabrication of the BG-TC device. (b) $I_{\mathrm{DS}}-V_{\mathrm{DS}}$ characteristic at different $V_{\mathrm{G}}$. (c) $I_{\mathrm{DS}}-V_{\mathrm{GS}}$ transfer curve at $V_{\mathrm{DS}}=-1 \mathrm{~V}$. Reproduced with permission from ref. 28 . Copyright 2016 American Chemical Society. 
data storage in the form of resistive random access memory (RRAM). Organic and hybrid nanomaterials are currently investigated for potential application in low-cost fabrication, flexible or transparent devices. ${ }^{339}$ Notably, immobilization of such materials in ZIF-8 films has also been explored as a route to facilitate their integration in resistive memories. ${ }^{340}$

Physical bipolar resistance switching in MOFs has currently only been observed for a single case. $\mathrm{Li}$ and co-workers reported the apparent coupling of ferroelectric behavior and resistance switching in single crystals of $\operatorname{In}[2 \text {-aminoterephthalate }]_{2}$ (coined resistance-switchable metal-organic framework 1 or RSMOF-1). ${ }^{39}$ In this study, a single crystal of RSMOF-1 was glued on a Pt substrate using silver colloidal paste and connected by a tungsten probe. The assembly showed two resistance states (ON/OFF ratio of 30) which could be reversibly switched at a relatively high threshold voltage of $\pm 7.5 \mathrm{~V}$. Polarization-electric field characterization of the crystal connected this behavior to its ferroelectric nature. It was hypothesized that the electrical properties of the MOF were strongly modulated by aligned water molecules densely packed in the one-dimensional nanopores. Molecular dynamics simulations showed that these guests hydrogen-bonded to the amine-lined pore walls undergo realignment upon polarization switching, resulting in a $0.16 \mathrm{eV}$ decrease of the activation energy for charge carrier hopping.

Chemical bipolar resistance switching assisted by MOFs was first demonstrated by Grzybowski and co-workers, who utilized Rb-CD-MOF (CD = cyclodextrine), a framework that contains mobile hydroxide ions in its pores. ${ }^{341}$ A single MOF crystal was contacted by applying silver colloidal paste at two opposite faces. Essentially, the MOF functions as a rigid selective electrolyte that allows hydroxide ions to pass but restricts mobility of the large hydrated silver ions released from the electrodes under anodic bias (Fig. 19). In this way, the MOF stabilizes the formation of a $\mathrm{AgOH} / \mathrm{AgO}_{x}$ passivation layer on the anode. The working principle of the device relies on the occurrence of negative differential resistance, which is a decrease of the current with increasing voltage due to formation of the resistive passivation layer. Writing was done at relatively high $\pm 10 \mathrm{~V}$ sweeps, reading at $2-4 \mathrm{~V}(\mathrm{ON} / \mathrm{OFF}$ ratio of 150$)$.

Chen and co-workers explored resistance-switching using ZIF-8 as porous dielectric. ${ }^{314}$ Patterned arrays of Si/ZIF-8/Ag memory cells were fabricated through room temperature solution deposition of $60 \mathrm{~nm}$ ZIF-8 films on doped silicon, followed by $\mathrm{Ag}$ thermal evaporation using a shadow-mask. When characterized as memristors at moderate voltages $( \pm 1-2.5 \mathrm{~V})$, the cells showed a relatively large sample-tosample distribution of the high and low resistance states, but consistently with a very large ON/OFF ratio of $10^{7}$. In this case, the mechanism of resistance switching relies on the reversible formation of silver ions at the silver electrode. These ions are transported to the counter electrode by electromigration in the applied field, and are reduced to silver nanoparticles. The conductive pathways that are formed between both electrodes in this way result in a transition from high to low resistance. Opposite biasing can be applied to reverse the process. This mechanism, filamentary switching, has been observed a

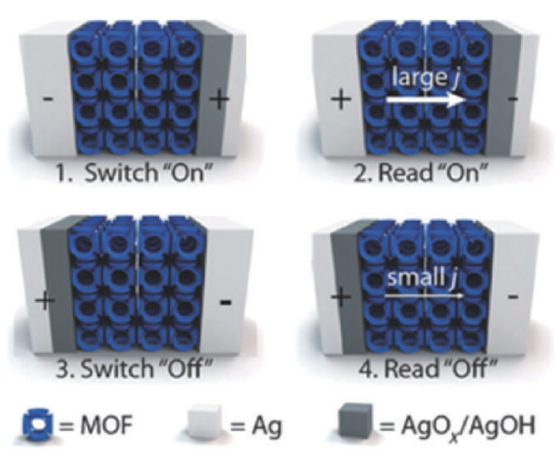

b

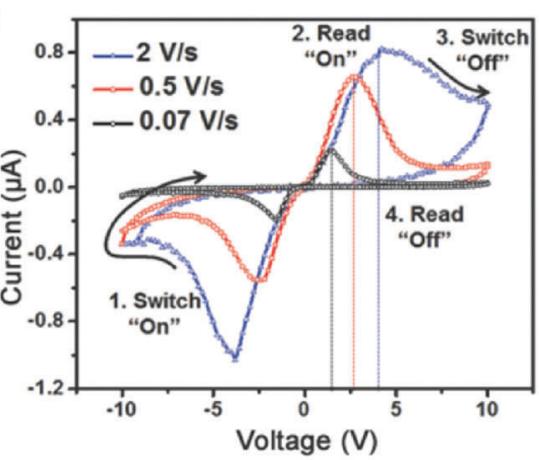

Fig. $19 \mathrm{Rb}$-CD-MOF electrolyte memristor. (a) Schematic representation of formation of the passivation layer during the different steps in operation of the device. (b) Cyclic voltammetry showed the occurrence of negative differential resistance. Adapted from ref. 341. Copyright 2014 Wiley \& Sons.

for many types of MIM systems. ${ }^{342}$ A distinguishing feature of the ZIF-8 matrix was investigated by operation of the device in atmospheres of different alcohols. These guest molecules were adsorbed in the ZIF-8 matrix and enable additional routes for electron-hopping and ionic conductance. As a result, environmental responsiveness and guest-modulated tuning of the current-voltage characteristics of the cells was demonstrated. Lastly, unchanged performance of similar cells integrated on flexible substrates was observed at different bending radii of the substrate (Fig. 20a).

In an analogous approach, $\mathrm{Li}$ and co-workers studied the resistive switching potential of Au/HKUST-1/Au/PET memory cells fabricated through $130 \mathrm{~nm}$ LPE growth of HKUST-1 SURMOF. ${ }^{315}$ An initial voltage sweep to $15 \mathrm{~V}$ was utilized as an in situ partial pyrolysis "forming" step, resulting in a switch from high to low resistance. Next, relatively small opposite sweeping voltages of $-0.5 \mathrm{~V}$ and $0.8 \mathrm{~V}$ could be utilized to induce switching (ON/OFF ratio of 18.5). A filament formation mechanism was also hypothesized in this study. In the initial step, copper ions are removed from the framework through electromigration while linkers are decarboxylated through Joule heating. These processes result in the formation of $\mathrm{sp}^{2}$-rich conductive filaments that can be reversibly disrupted and formed through subsequent writing and erasing operations. The approach was reproducible over at least 50 samples and in a broad temperature range between -70 and $70{ }^{\circ} \mathrm{C}$. In addition, only a minor influence of bending the flexible device was found (up to $2.8 \%$ tensile strain), for over a hundred of repeated 
a,i

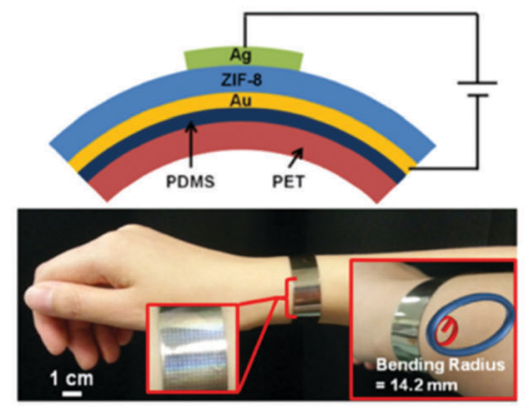

b,i

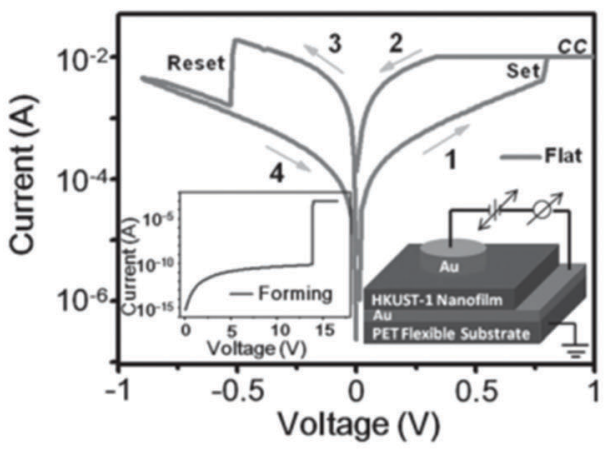

a,ii

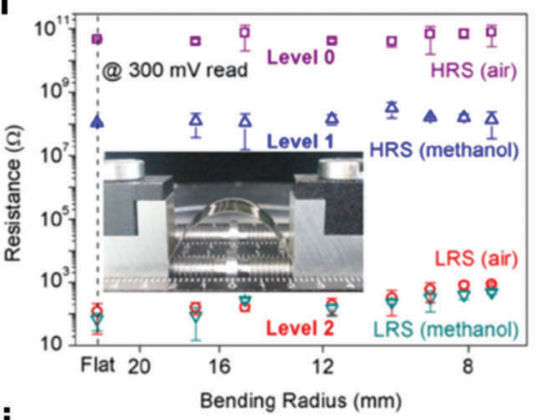

b,ii

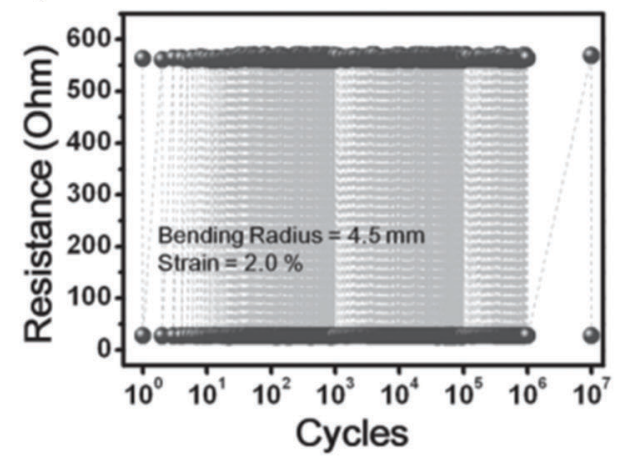

Fig. 20 Examples of integration of MOFs on flexible substrates for application in resistive memory. (a,i) Schematic representation of Ag/ZIF-8/Au memristor and demonstration of its implementation at the $14.2 \mathrm{~mm}$ bending radius of a wearable wristband. (a,ii) High and low resistance states (HRS, LRS) of the device at different bending radii and upon exposure to methanol vapor. Inset: Picture of the bending test experimentation. Reproduced with permission from ref. 314. Copyright 2016 Wiley \& Sons. (b,i) Operation window of Au/HKUST-1/Au memristor. Insets are the device schematic and the breakdown current during the "forming" step. (b,ii) Stable switchable states of the device over $10^{7}$ cycles at 4.5 mm bending radius. Reproduced with permission from ref. 315. Copyright 2015 Wiley \& Sons.

tests. The authors point out that this performance is by far superior to purely inorganic counterparts. Moreover, the performance level was maintained for $10^{7}$ cycles at $2 \%$ tensile strain (Fig. 20b).

Redel and co-workers investigated a similar system consisting of SURMOF films integrated by LPE in Au/HKUST-1/Al/Au sandwich structures. ${ }^{343}$ These ultrathin film devices showed switching behavior at low potentials $( \pm 1 \mathrm{~V})$ without the need for an initial pyrolysis step, and in the absence of evidence for copper migration or framework decomposition. Performance fine-tuning of the device was shown through a downsizing approach (from 50 down to $10 \mathrm{~nm}$ ) and by loading the SURMOF with ferrocene guest molecules. Although the underlying mechanism for this behavior is not well understood, an interesting clue comes from recent work by Attfield, Dryfe and co-workers, who produced HKUST-1 coated $\mathrm{Cu}$ electrodes via an anodic dissolution process. ${ }^{344}$ In this study, the observed data storage behavior is attributed to electrically accessible $\mathrm{Cu}$ cations within the MOF that are able to undergo facile $\mathrm{Cu}(\mathrm{II}) / \mathrm{Cu}$ (I) redox interconversion as a function of the applied potential. Optimization of electrolyte composition and concentration and electrode separation resulted in an ON/OFF ratio of $\sim 5$, with each state stable to at least 10 consecutive reads and rewritability that persists over 6000 cycles.

\subsection{Chemical sensors}

Chemical sensors are devices that respond to changes in analyte concentration and transduce this information as electrical signals. Such measurement and monitoring devices are crucial for instance in industrial leakage testing and household carbon monoxide detectors. Improvements in terms of performance, size and cost would open up a range of new opportunities for chemical sensors. For example, more energyefficient and better insulated homes will benefit from sensorcontrolled ventilation systems to ensure indoor air quality. ${ }^{345}$ Exciting prospects also exist in medical diagnostics, through detecting organic tracer molecules in human breath. ${ }^{346}$ In addition, major demand is building for performant, small, cheap and robust sensors that enable integration in wearables and mobile phones, therefor hinting at distributed sensor networks. $^{347}$ In this context, fabrication scalability and low power consumption are requirements to take into account.

Key performance characteristics of chemical sensors are sensitivity (i.e. detection limit), stability (i.e. drift, reversibility) and selectivity (i.e. no cross-analyte response). While sensitivity and to some extent stability depend on the sensor type, peripheral electronics and signal processing (e.g. amplification and noise filtering), selectivity more directly depends on the performance of the sensing material capturing and interacting with the analyte. Although approaches such as sensor arrays address this challenge to some extent, selectivity arguably remains the biggest challenge in currentgeneration detection and monitoring devices. In comparison to non-ordered sensing materials (e.g. polymer coatings), MOFs offer improvements in selective analyte recognition through precisely 


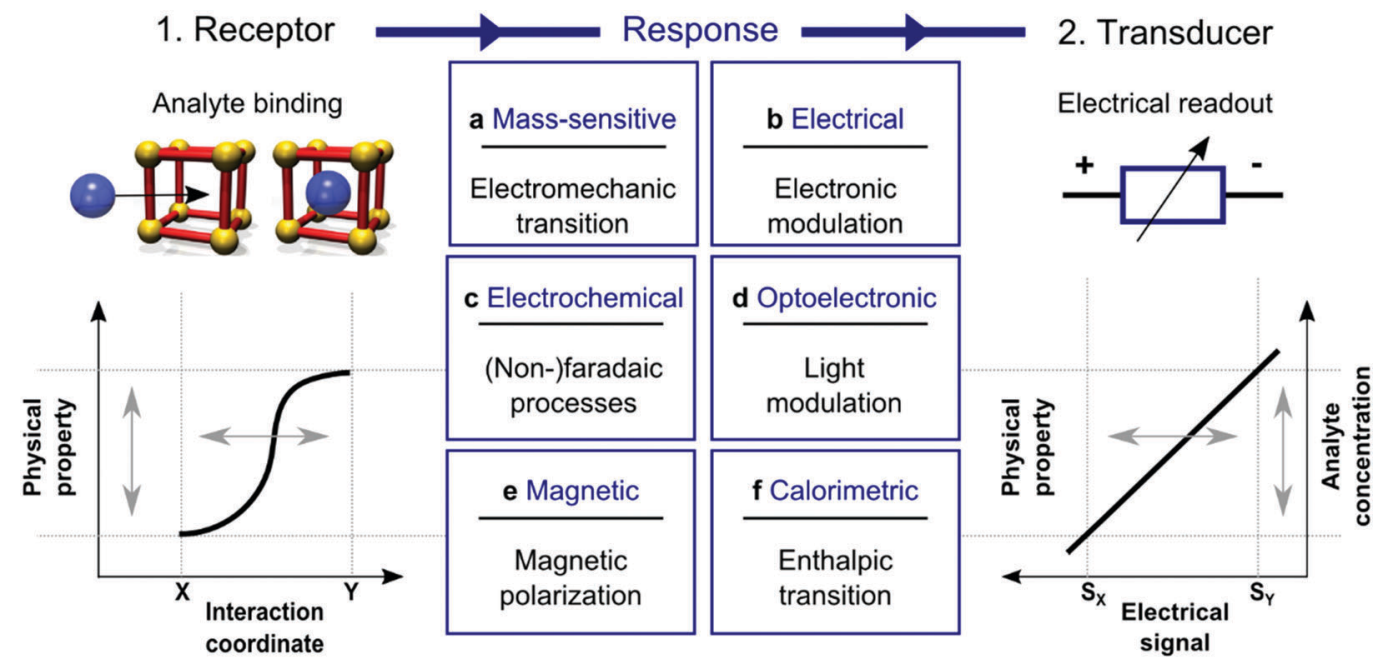

Fig. 21 Electronic chemical sensors. Schematic overview of widespread strategies $(a-f)$ for chemical-to-electrical transduction. Two functions are distinguished: the receptor, and the transducer. The first element conducts the chemical-to-physical, the second the physical-to-electrical transduction. Ideally, the resulting electrical signal can be (linearly) correlated to the concentration of a targeted analyte.

tuned pore environments and molecular sieving. In our overview, MOF chemical sensors are classified according to the transducer operating principle as shown in Fig. $21 .^{348,349}$

4.2.1 Mass-sensitive sensors. Mass-based sensors transduce the uptake of an analyte into a mechanical response (e.g. bending or changes in vibration behavior) that can be detected as an output signal, often through piezoelectric materials. Microcantilevers, microresonators, and acoustic wave sensors, for example, are highly sensitive microelectromechanical systems (MEMS) that can monitor mass changes in an adsorbent film. For polymer-functionalized mass sensors it is often a challenge to achieve high selectivity for the analyte of interest. Approaches include utilizing an array of partially selective films or coupling the sensor with a preceding separation step, such as gas chromatography. MOFs could address this problem by serving as a highly selective sorbent layer. The discussion below introduces the operating principles of these MEMS sensors and highlights selected instances where MOFs have been integrated.
Microcantilevers. Microcantilevers perform as highly sensitive mass sensors by one of two transduction mechanisms. ${ }^{350}$ Dynamic microcantilevers detect changes in the resonant frequency of an oscillating microfabricated cantilever beam upon analyte adsorption (Fig. 22). Static microcantilevers, on the other hand, sense mass uptake by stress induced at the interface between the beam surface and an applied coating, causing the microcantilever to bend. Bending can be quantified by optical reflection using a diode laser and a linear position sensitivedetector, or alternatively, via a piezoresistive strain gauge built into the device. Both designs can be used for a variety of physical, chemical, and biological sensing applications, ${ }^{351-353}$ and arrays of microcantilevers have been proposed to increase both measurement speed and selectivity. ${ }^{354}$

In contrast to inorganic porous materials such as zeolites, MOFs are particularly attractive as analyte recognition layers for static microcantilevers, as they can exhibit large changes in crystal lattice parameters upon guest adsorption, resulting in

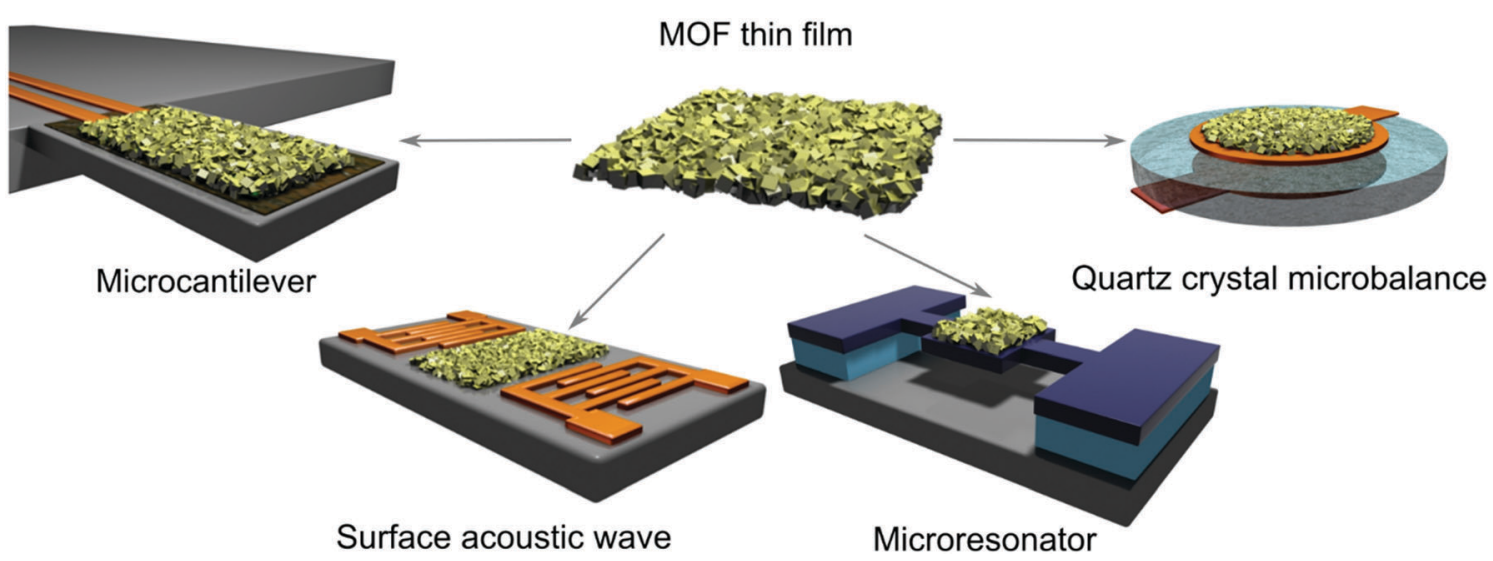

Fig. 22 Schematic overview of the four basic classes of representative mass-sensitive transducer platforms in which MOFs have been incorporated as thin film adsorption receptor layers. 
detectable mechanical strain. This concept was first exploited to detect water vapor using HKUST-1 thin films on a silicon microcantilever with a built-in piezoresistive sensor. ${ }^{355}$ Highly sensitive and rapid detection of volatile organic compounds was subsequently demonstrated. ${ }^{355-359}$ The sensing of alcohol vapors using $\mathrm{ZIF}-8^{360}$ and $\mathrm{CO}_{2}$ using MIL-53(Al) ${ }^{361}$ was more recently demonstrated through the simultaneous measurement of both resonance frequency and deflection changes on silicon cantilevers. From a device design standpoint, multiphysics materials modeling pointed out that increasing the Young's modulus of the MOF thin film increases the sensitivity of the functionalized microcantilevers, even more so than the density or Poisson's ratio. ${ }^{362}$

Although these initial studies were performed using thin films of relatively rigid MOFs, frameworks with greater adsorption-induced structural flexibility have been reported in the literature. For example, the structural distortions exhibited by HKUST- $1^{363}$ are minor compared to the large-scale volume changes $(\sim 40 \%)$ exhibited by MIL-53(Cr) in response to varying water contents. ${ }^{364}$ By exploiting such highly flexible materials, microcantilevers with unprecedented sensitivities for target analytes can be envisioned. To guide the selection of suitable materials, a deeper understanding of MOF mechanical properties and their relationship to framework dynamics upon guest adsorption is needed. A theoretical framework for rationalizing such behavior is being actively developed, ${ }^{365}$ but is still in its early stages. An additional challenge is that the vast majority of characterization studies needed to identify suitable MOF candidates were performed using bulk powder samples. Recent work demonstrates that a MOF that is rigid and non-porous in particle format can exhibit dynamic, gate-opening type behavior when downsized to a thin film. ${ }^{366}$ It is not yet clear how common such downsizing effects are among MOFs, or what the range of magnitudes may be. However, related work concerning crystal size effects also shows that these can significantly affect the phase transition behavior of ZIF-8. ${ }^{367}$ These studies suggest that care should be taken before using the properties and behavior of bulk MOFs to predict the properties of their thin film state.

Microresonators. Yaghi, Candler and co-workers fabricated ZIF-69 resonant sensors that are conceptually similar to dynamic microcantilevers, except with a design involving torsional beams supported by a wide center paddle region. ${ }^{242,368}$ In an elegant demonstration of MEMS fabrication, these microresonators were formed via a multi-step process involving $\mathrm{SiO}_{2}$ and polysilicon deposition followed by patterning and chemical etching steps. ZIF-69 particles were then deposited onto the paddle using either drop-casting ${ }^{368}$ or dielectrophoresis. ${ }^{242}$ The ZIF-69 microresonators were used for the detection of $\mathrm{CO}_{2}$ and isopropyl alcohol by laser Doppler vibrometry, and achieved a mass detection limit of $\sim 26 \mathrm{fg},{ }^{242}$ a value comparable to that of static microcantilevers (Table 2). Eddaoudi, Younis and co-workers integrated HKUST-1 films on electrostatically actuated resonant polymer microbeams designed to display a mass-sensitive natural frequency and pull-in frequency band (i.e. range at which the
Table 2 Typical operating frequencies and mass detection limits for representative mass-sensitive sensor designs. ${ }^{372,392}$ Note that actual sensor performance can be highly dependent upon additional factors such as the substrate thickness, sensor exposure conditions, and physical properties (thickness, uniformity, adhesion) of the thin film

\begin{tabular}{lll}
\hline Device & $\begin{array}{l}\text { Operating frequency } \\
(\mathrm{MHz})\end{array}$ & $\begin{array}{l}\text { Mass detection } \\
\text { limit }(\mathrm{ng})\end{array}$ \\
\hline Microcantilever (oscillating) & $\sim 0.02-5$ & $\sim 0.01$ \\
Microcantilever strain gauge & N/A & $10^{-6}$ \\
Quartz crystal microbalance & $\sim 5-30$ & $\sim 1$ \\
Surface acoustic wave sensor & $\sim 20-500$ & $\sim 0.1$
\end{tabular}

resonator collapses with the counter electrode). ${ }^{369,370}$ The MOF was integrated on the microfabricated device through inkjet printing for the detection of water and ethanol. In this way, it was shown that these sensors were sensitized for binary switching between a "safe" and "alarm" signal, whereas the resonance amplitude could be related to the gas concentration down mass detection limit of $\sim 371 \mathrm{pg}$.

QCM devices. Acoustic wave sensors rely upon adsorbateinduced frequency changes of waves propagating either through or across surfaces, typically at $\mathrm{MHz}$ frequencies, resulting in an electrical excitation that can be detected in piezoelectric materials. Quartz crystal microbalances (QCM) and surface acoustic wave (SAW) sensors, are two of the most commercially developed acoustic devices and have been integrated with MOF thin films for sensing applications. QCM devices generally consist of an AT-cut quartz substrate with circular electrodes patterned on both sides. By applying a voltage between the electrodes, the piezoelectric properties of the quartz crystal create a shear deformation. By applying an alternating voltage, the QCM crystal operates as part of an oscillator circuit that changes frequency upon guest adsorption on the MOF-functionalized top electrode. Assuming the MOF thin film is rigidly adhered to the resonator surface and has acoustical properties identical to quartz, the decrease in resonant frequency upon mass adsorption can be approximated by the Sauerbrey eqn (5): $:^{371}$

$$
\Delta f=-\frac{2 f_{0}^{2}}{A\left(\mu_{\mathrm{q}} \rho_{\mathrm{q}}\right)^{1 / 2}} \Delta m
$$

in which $\Delta f$ is the frequency shift due to added mass, $f_{0}$ is the resonant frequency, $A$ is the area of the quartz crystal between the electrodes, $\mu_{\mathrm{q}}$ is the shear modulus of the quartz substrate, $\rho_{\mathrm{q}}$ is the density of quartz, and $\Delta m$ is the mass change. Typical QCM devices operate at frequencies between 5-30 MHz (Table 2), and for a device with an upper frequency of $\sim 10 \mathrm{MHz}$ a quartz thickness of around $150 \mu \mathrm{m}$ is needed. ${ }^{372}$ Although decreasing the crystal thickness would increase the device operating frequency (and thus its mass sensitivity), this would make the device too fragile to fabricate or handle.

Two factors must be taken into account in determining the appropriate thickness of MOF films in QCM devices. The first consideration applies irrespective of the type of sensing device and concerns the sensor response time upon exposure to an 
analyte. Heinke et al. quantified this relationship and showed that although the cyclohexane loading in a HKUST-1 SURMOF film was unaffected by its thickness (ranging from 52 to $690 \mathrm{~nm}$ ), the time to reach full-scale response (i.e. equilibrium) increased quadratically with thickness. ${ }^{373}$ This observation indicates that the uptake for SURMOF films is limited by intra-crystal diffusion rather than a surface energy barrier. The second consideration is the validity of the Sauerbrey equation. As mentioned above, a key assumption is that the film has identical acoustical properties as the quartz crystal, which is critically related to the acoustic wave coupling at the substrate-film interface. This is governed by the film thickness, but also by the film adhesion and the differences in mechanical properties of the MOF relative to quartz. These relationships are poorly understood for most materials; regarding MOFs, a systematic investigation of device response versus film thickness would be of great value.

An advantage of QCM over dynamic microcantilevers is facile operation under vacuum, gas, or liquid-phase conditions. This versatility, combined with their simplicity and commercial availability, have made these devices widely used tools for in situ probing of a wide array of MOF film properties. The chemical affinity of MOFs for adsorptive chemical sensing of various volatile organics has been studied using thin films deposited on QCM. ${ }^{199,221,359,374,375}$ The methodology was utilized in a few cases to study the substantial influence of structure and chemical functionalization of multicomponent thin films on the sensing selectivity. ${ }^{376-380}$ In addition, QCM has been frequently used as a tool to study transient phenomena ranging from structural responsiveness and mass transfer kinetics of guests in MOF thin films, ${ }^{366,373,381-385}$ to real-time insight into the mechanisms of layer-by-layer growth during the thin film deposition process. ${ }^{195,386-388}$

$S A W$ devices. Another acoustic wave device commonly used for chemical sensing applications is the surface acoustic wave (SAW) sensor. First reported as a chemical vapor sensor in $1979,{ }^{389}$ a key distinction between SAW and QCM devices is the acoustic modes by which they operate. Whereas QCM devices utilize shear horizontal waves, a SAW device primarily relies upon surface-normal displacements that create what is known as a Rayleigh wave. ${ }^{390}$ One implication of this operating mode is that, although SAW devices can operate at much higher natural frequencies (tens of $\mathrm{MHz}$ to $\mathrm{GHz}$ ) than QCM devices, thereby endowing higher mass sensitivity, these compressive Rayleigh waves cannot propagate effectively into liquids. Consequently, SAWs do not operate efficiently outside of gaseous or vacuum environments. For a comparison of the typical operating frequencies and gravimetric sensitivities of the acoustic wave sensors discussed in this section, see Table 2 .

Like QCMs, SAW devices are also commercially available, but have been less explored for sensing applications using MOF films despite their exceptional mass sensitivity. Nevertheless, this high sensitivity was exploited to create a SAW humidity sensor based that exceeded the sensitivity of many commercially available sensors (sub-ppm concentrations) by coating ST-cut quartz operating at $97 \mathrm{MHz}$ with HKUST-1. ${ }^{391}$ In a more recent study, the use of
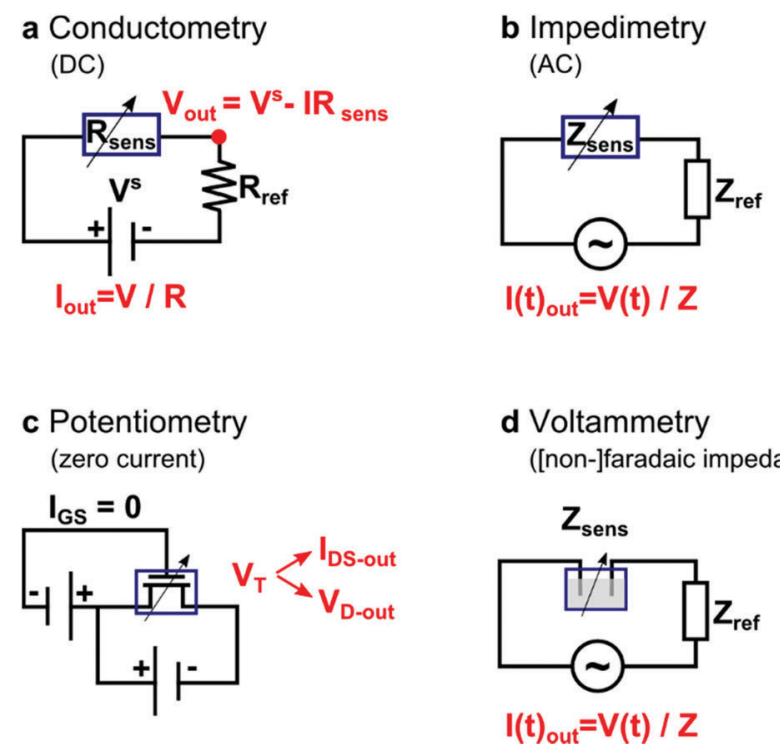

Fig. 23 Overview of some relevant electrical and electrochemical sensor circuits. The position of the chemically sensitive MOF is represented by the blue square (and black arrow to indicate external modulation of the $1-V$ characteristics). Selected examples of the output signals are shown. (a) Conductometry. (b) Impedimetry or impedance spectroscopy. (c) Potentiometry. (d) Voltammetry or electrochemical impedance spectroscopy.

MOF-based SAW sensors was extended to include sensing of volatile organic compounds using thin films of various MOFs sharing the " $n b o$ " topology as the chemical recognition layer. ${ }^{388}$

4.2.2 Electrical and electrochemical sensors. Electrical and electrochemical chemical sensors are based on direct chemical-toelectrical signal transduction. However, the working principle of these devices is based on different physicochemical mechanisms that affect electronic properties such as charge density, charge mobility, polarizability and electrostatic potential. As a general operation principle, the current-voltage $(I-V)$ characteristic of a chemically sensitive electrical component changes in relation to the analyte concentration and is determined through circuit analysis (Fig. 23). Depending on the ideal behavior of this component, such sensors are typically categorized as chemiresistors, chemicapacitors, chemitransistors, etc.

Conductometry and chemiresistors. Chemical sensors operating through analyte-induced electrical conductance variation are often analyzed through monitoring of the direct current (DC) that flow through the element at a constant biasing voltage (i.e. conductometry). The specific conductance or conductivity $(\sigma)$ of a material is the ratio of the current density $\left(j, \mathrm{~A} \mathrm{~cm}^{-2}\right)$ and the electric field $\left(E, \mathrm{~V} \mathrm{~cm}{ }^{-1}\right)$. For a mixed semiconductor, the conductivity is determined by the mobility $(\mu)$ and density $(N)$ of holes (p) and electrons (n), as given by the general conductivity eqn (6). ${ }^{393}$

$$
\sigma=\frac{j}{E}=e \mu_{\mathrm{p}} N_{\mathrm{p}}+e \mu_{\mathrm{n}} N_{\mathrm{n}}
$$

Hence, the conductivity is changed by those physicochemical interactions between the analyte and the sensing material that 
alter either the concentration of charge carriers or their mobility. The resistance (i.e. inverse of conductance) of an unknown circuit element can be analyzed directly from the current flowing through the elements in a connected known circuit. As the working principle of a chemiresistor relies on its ohmic behavior, at least moderate electrical conductance of the sensor assembly is required; otherwise no observable current and voltage drop would arise over the sensor. Semiconductor metal oxide (SMO) sensors based on wide band gap ceramics such as zinc oxide, tin oxide and tungsten oxide are the most widespread commercial chemical sensors. SMO sensors have to be operated at high temperature $\left(>200-400{ }^{\circ} \mathrm{C}\right)$, as conductance shifts are caused by the formation of surface states by chemisorption of oxidizing or reducing gases at the gas-solid interface. These surface states alter the conductance of the space charge layer at the oxide grain boundaries. Molecular semiconductors such as conductive polymers, ${ }^{394}$ and carbon allotropes such as graphene ${ }^{395}$ and carbon nanotubes, ${ }^{396}$ offer alternative operating principles at room temperature, as relatively weak charge transfer interactions have been observed to lead to significant conductance shifts. However, these materials typically need extensive functionalization to achieve high sensitivity towards targeted analytes.

A first share of studies, recently published in parallel by different groups, investigated the performance MOF@SMO heterostructures as chemiresistors. In these devices, the MOF functions as receptor material, whereas conductance and chemiresistive behavior is supported by the SMO. The main motivation for this approach is the limited selectivity of SMO sensors, which leads to high cross-sensitivity. In a first study by Yao et al., ZnO nanowires were hydrothermally deposited on a seeded sapphire substrate. Subsequently, ZIF-8/67@ZnO nanowire heterostructures were fabricated by solvothermal treatment of the structures with a precursor solution (Fig. 24a). ${ }^{397}$ Colloidal silver paste was used to create ohmic contacts at two ends of the substrate. A catalytic effect of the Co-based ZIF-67 in acetone sensing was observed at $260{ }^{\circ} \mathrm{C}$, in comparison with reference measurements with non-coated nanowires (tenfold higher signal). Acetone sensing was successfully demonstrated between 0.25 and $100 \mathrm{ppm}$, showing a $2 \mathrm{ppb}$ extrapolated detection limit based on the signal noise level. In contrast, the sensor was fully desensitized to humidity variations due to the hydrophobic nature of the MOF (Fig. 24a), showing feasibility of the device for selective acetone trace detection in a humid background, similar to the conditions in diagnostic breath analysis. In a second study by Drobek et al., ZIF-8@ZnO nanowire heterostructures were fabricated by vapor deposition of the nanowires on interdigitated electrode (IDE) substrates, followed by solvothermal treatment with a precursor solution to form the MOF shell. ${ }^{209}$ Conductometric chemical sensing was performed at $300{ }^{\circ} \mathrm{C}$. The results showed overall reduced sensitivity (various gases/vapors at $10-50 \mathrm{ppm}$ ) in comparison to the pristine nanowires, but significantly improved selectivity towards hydrogen ( $0.29 \mathrm{~nm}$ kinetic diameter) over larger molecules such as benzene and toluene (0.52-0.53 nm kinetic diameter, respectively). This effect was attributed to the narrow pore aperture (0.34 $\mathrm{nm}$ diameter) of the ZIF-8 framework. Therefore, these results are an exciting demonstration of size exclusion through molecular sieving for chemical sensing applications. Lastly, in a third related study by Tian et al., ZIF-8@ZnO nanorod heterostructures were fabricated by solvothermal processing as a powder and subsequent spin coating from a suspension on an

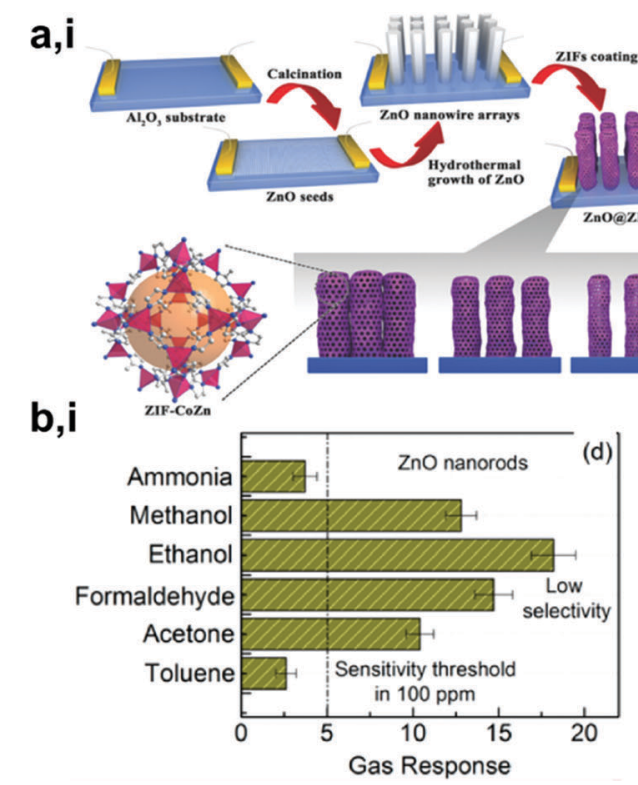

\section{a,ii}

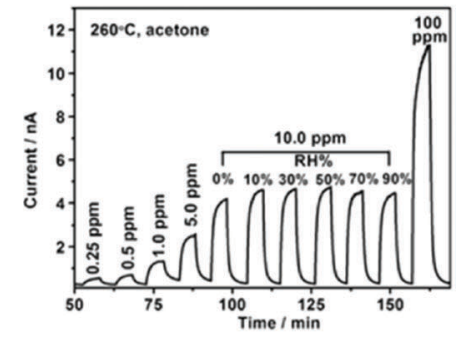

b,ii

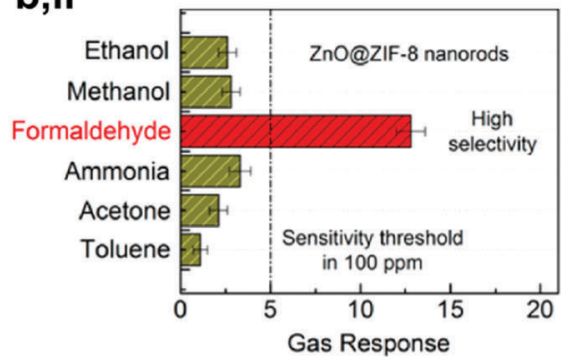

Fig. 24 MOF-SMO heterostructures as chemiresistors. (a,i) Schematic representation of ZIF-8-ZnO nanowire SMO fabrication through seeded deposition and solvothermal treatment with the linker. (a,ii) Chemiresistive sensing experimentation showed that acetone detection at 10 ppm was made insensitive to variation of the humidity. Adapted with permission from ref. 397. Copyright 2016 Wiley \& Sons. (b,i) ZnO nanorod SMO, and (b,ii) ZIF$8-\mathrm{ZnO}$ nanorod response to various volatile organics, showing ZIF-8-induced selectivity towards formaldehyde over more polar analytes and analytes with a larger kinetic diameter. Adapted with permission from ref. 398. Copyright 2015 American Chemical Society. 
alumina substrate with pre-integrated resistive heater and gold contacts. $^{398}$ Conductometric sensing experiments at $300{ }^{\circ} \mathrm{C}$ showed a quasi-linearly correlated response of the device towards formaldehyde at concentrations between 10 and 200 ppm (extrapolated detection limit $5.6 \mathrm{ppm}$ ). Moreover, the interfering signals of more polar analytes such as water, methanol and ethanol, and that of analytes with a larger kinetic diameters such as ammonia, acetone and toluene, was significantly reduced by the ZIF-8 shell (Fig. 24b). The previous studies show the advantageous properties of MOFs as tailorable receptor materials to improve the selectivity of SMO chemiresistors. Importantly, heterostructured sensors of this type need to be operated at relatively high temperature due to the SMO transduction mechanism.

MOF sensing layers can be combined with materials other than SMOs to fabricate chemiresistors that operate at much lower temperatures. For example, HKUST-1@graphene oxide composite devices were fabricated by slurry blade-casting on IDE substrates. ${ }^{399}$ By pretreatment in ammonia, the MOF was converted to an amorphous phase that demonstrates reversible binding of ammonia. The MOF-derived device showed a quasilinear response to ammonia concentrations between 100-500 ppm at room temperature. However, the response of the system was rather low in comparison to other functionalized graphene sensors. Much improved responses may be expected for MOFs that can maintain their crystallinity and porosity upon exposure to the analyte. An interesting variation on coating conductive materials with MOFs is to grow conductive polymers in the MOF pores. Le Ouay et al. grew conductive PEDOT chains inside MIL-101 particles and created a resistive $\mathrm{NO}_{2}$ sensor by drop casting this composite on an IDE. ${ }^{400}$ Although the surface area of the composite $\left(S_{\mathrm{BET}}=1038 \mathrm{~m}^{2} \mathrm{~g}^{-1}\right)$ is lower than that of the pristine MOF, the pores provide much improved accessibility to the polymer chains compared to bulk PEDOT $\left(S_{\mathrm{BET}} \approx 2 \mathrm{~m}^{2} \mathrm{~g}^{-1}\right)$. The nanoscale structuring of the polymer resulted in a linear response at room temperature up to $10 \mathrm{ppm}$ of $\mathrm{NO}_{2}$, with rapid equilibration times $(<30 \mathrm{~s}$ for low concentrations) and an extrapolated detection limit of $60 \mathrm{ppb}$.

Other studies have demonstrated the application of MOFs directly as intrinsically chemiresistive materials. In these examples, the MOF material functions both as receptor and transduction material. Zhang and co-workers reported the chemiresistive behavior of $\mathrm{ZIF}-67^{401}$ and $\mathrm{Co}(\mathrm{IM})_{2}{ }^{402}$ films fabricated by slurry casting on IDE substrates. The ZIF-67 device was used in formaldehyde sensing at $150{ }^{\circ} \mathrm{C}$ and showed significant responses for concentrations between 5 and $500 \mathrm{ppm}$. A limited influence of humidity was observed, as expected for the hydrophobic ZIF-67 framework. The $\mathrm{Co}(\mathrm{IM})_{2}$ device was employed at $75{ }^{\circ} \mathrm{C}$ for trimethylamine (TMA) sensing between 2 and $500 \mathrm{ppm}$. Strong TMA adsorption was indicated by the slow recovery time $(\sim 1 \mathrm{~h})$. By contrast, negligible response of the device was demonstrated for water, methane, acetone, ammonia and methanol. The origin of this selectivity is unclear. The authors attributed the chemiresistor performance of the $\mathrm{Co}$ (II)imidazolate frameworks to the low band gaps of $1.98 \mathrm{eV}$ and $1.80 \mathrm{eV}$. However, as conduction mechanisms and experimental conductivities were not reported, further investigation is needed to understand the "turn-off" conductance shifts of these sensors. Dincă and coworkers demonstrated chemiresistive ammonia sensing for the conductive $\mathrm{Cu}_{3}(\mathrm{HITP})_{2}$ MOG ( $0.2 \mathrm{~S} \mathrm{~cm}^{-1}$ bulk conductivity) by drop casting the material on IDE substrates. ${ }^{71}$ The device showed a quasilinear and reversible "turn-on" response between 0.5 and 10 ppm ammonia at room temperature. To simulate real-world conditions, quantitative ammonia monitoring was demonstrated down to $5 \mathrm{ppm}$ in air at $60 \%$ relative humidity $(\mathrm{RH})$, which is competitive with literature benchmarks. In follow-up work, a sensor array was prepared consisting of three IDE substrates coated with the $\mathrm{Cu}_{3}(\mathrm{HITP})_{2}$ and two isomorphs with altered linkers and metal centers, $\mathrm{Cu}_{3}(\mathrm{HHTP})_{2}$ and $\mathrm{Ni}_{3}(\mathrm{HITP})_{2} \cdot{ }^{403}$ Monitoring the response of each sensor upon exposure (200 ppm, room temperature) to five classes of volatile organic compounds or VOCs indicated that the nature of the metal center, $\mathrm{d}^{8} \mathrm{Ni}$ (II) versus $\mathrm{d}^{9} \mathrm{Cu}$ (II), had the largest impact on the signal intensity. As the charge density of the MOG is considerably affected by the metal center, this indicates that electron transfer between the analytes and the MOG nodes is probably responsible for the chemiresistive response. However, the results showed clear evidence for multiple sensing mechanisms with different concentration-dependence. By combining the response of the three MOG-based chemiresistors, the study showed how it is possible to distinguish between VOC categories through principal component and linear discriminant analysis (Fig. 25).

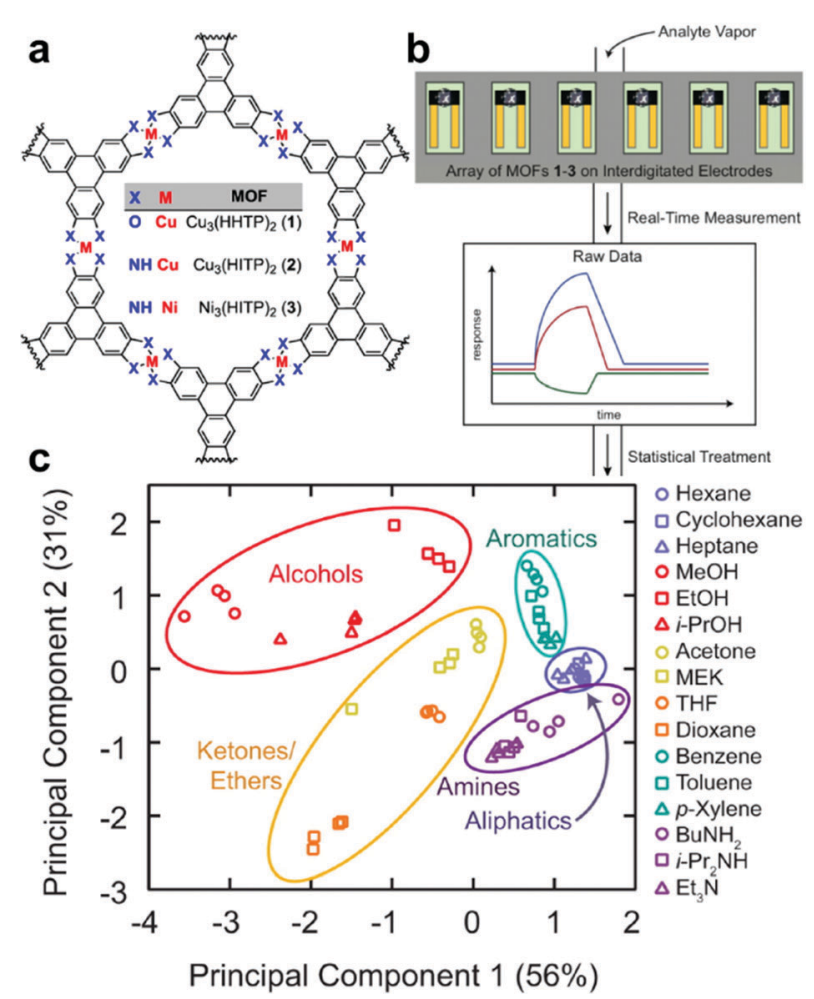

Fig. 25 MOGs as intrinsic chemiresistors. (a) Structure of three isoreticular analogues of $\mathrm{Cu}_{3}(\mathrm{HITP})_{2}$ that were utilized in the sensing array. (b) Schematic representation of the measurement of the sensing responses of the different IDE sensors, followed by statistical treatment of the data. (c) Clustering of different VOC categories based on principal component analysis of the signals from the array of three sensors. Adapted with permission from ref. 403. Copyright 2015 American Chemical Society. 
Impedimetry and chemicapacitors. A very general readout strategy for two-terminal chemical sensors is through monitoring of the current-voltage behavior in the frequency domain, by connecting the device to an alternating current (AC) power source. In AC circuit analysis, the generalized concept of impedance $(Z, \Omega)$ is defined in analogy to DC resistance, as the voltage to current ratio. The real part of the impedance is the resistance $(R)$ and the imaginary part is the capacitive reactance $\left(X_{\mathrm{C}}\right)$, which depends on the electric field geometry (7). $X_{\mathrm{C}}$ is inversely proportional to the dielectric constant $(\kappa)$ of the sensing material (8), and hence the variation of this property upon analyte adsorption can be exploited for chemical sensing.

$$
\begin{gathered}
Z=\frac{V(t)}{I(t)}=R+j X_{\mathrm{C}} \\
X_{\mathrm{C}} \propto \frac{1}{\kappa}
\end{gathered}
$$

Note that the resistance does not contribute to the complex term of the impedance. In other words, electric charge transport through the MOF is not required for chemicapacitors analyzed in the frequency domain. The required readout and data processing circuitry are generally more complex for chemicapacitors than for chemiresistors. Conversely, the biasing frequency is an additional experimental variable ( $c f$. impedance spectroscopy), offering opportunities for elucidation of the host-guest interactions through transient circuit analysis. ${ }^{393}$ Additionally, the absence of a net polarization of the contacts eliminates interference of the contact resistance with the analysis.

The early proof-of-concept chemicapacitor by Moos and coworkers (2009) was one of the first examples of application of MOFs in an electronic device. ${ }^{404}$ A set of commercial MOF powders (BASF Basolite) was casted together with an organic binder on IDE substrates. Impedimetry at $1 \mathrm{~Hz}$ and $120{ }^{\circ} \mathrm{C}$ showed a quasi-linear and reversible response to water for MIL-100(Fe), of $590 \mathrm{M} \Omega$ per \% RH up to $2.5 \%$. In the same study, the feasibility of impedimetric VOC sensing was demonstrated as well. Ruan and co-workers, more recently reported a similar approach for water sensing at higher concentration using MIL-125(Ti)- $\mathrm{NH}_{2}$ slurry casted on IDE substrates. ${ }^{405}$ Measurements at room temperature and $100 \mathrm{~Hz}$ showed a quasi-linear response to humidity of roughly $1.4 \mathrm{M} \Omega$ per \% $\mathrm{RH}$ between 11 and 95\% RH. Impedance spectroscopy showed that the device output became independent of $\mathrm{RH}$ at $\mathrm{kHz}$ frequencies, indicating the possible role of ionic transport of dissociated water in its impedimetric detection in MIL-125(Ti)- $\mathrm{NH}_{2}$. In another example, Qiu and co-workers evaporated millimeter-sized aluminum dots on a HKUST-1 film solvothermally grown on polished copper. ${ }^{406}$ The resulting parallel-plate chemicapacitors showed a linear, reversible capacitance response to humidity at $1 \mathrm{MHz}$ of approximately $1.5 \mathrm{pF}$ per \% RH between 10 and 90\% RH. In a later study, Zeinali and Homayoonnia showed a link between the HKUST-1 crystallite size and the response kinetics of similar parallel-plate chemicapacitors. ${ }^{407}$ Wagner and co-workers fabricated parallel-plate chemicapacitors via deposition of gold electrodes on both sides of pressed pellets of different isoreticular analogues of aluminum-isophthalate CAU-10. ${ }^{408,409}$
These assemblies were operated as humidity sensors at room temperature and the $\mathrm{mHz}-\mathrm{MHz}$ spectral range. Using the Havriliak-Negami model, different non-correlated contributions were distinguished for the observed impedance, such as a 5 orders of magnitude variation of the DC resistance over the humidity range. However, the observed response of $0.13 \mathrm{pF}$ per $\% \mathrm{RH}$ was rather low. In a later study, the occurrence of a phase transformation was observed in CAU-10 during hydration and dehydration, ${ }^{410}$ an effect that can enhance either counter balance the polarizability rises that are anticipated for water uptake in the pores. The rather low response in the study might indicate the latter effect.

De Smet and co-workers recently reported fabrication of a microelectronic sensor by drop casting MIL-53- $\mathrm{NH}_{2}(\mathrm{Al})$ nanoparticles in a polymer matrix (MOF@polymer) on top of a capacitance transducer manufactured through $140 \mathrm{~nm}$ CMOS processing (Fig. 26a). ${ }^{411}$ The active area of the device consisted

a
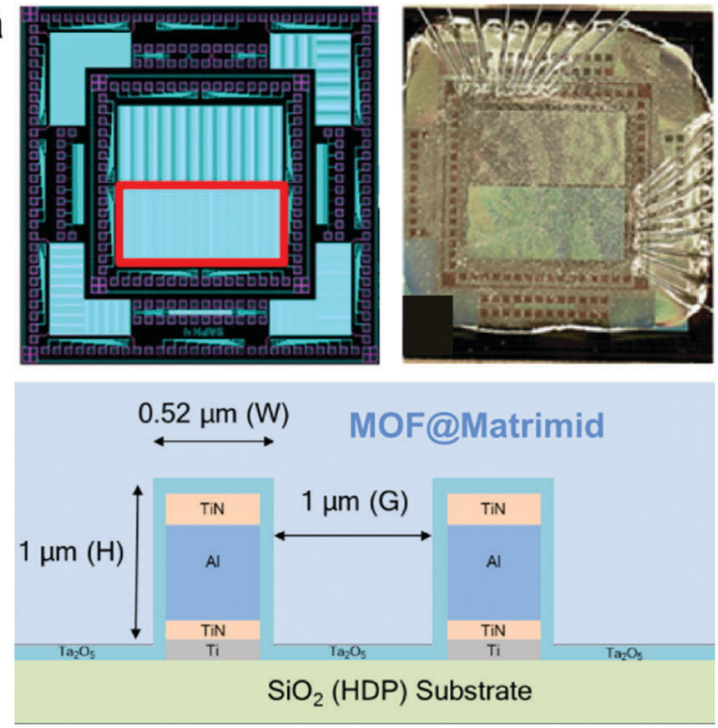

b

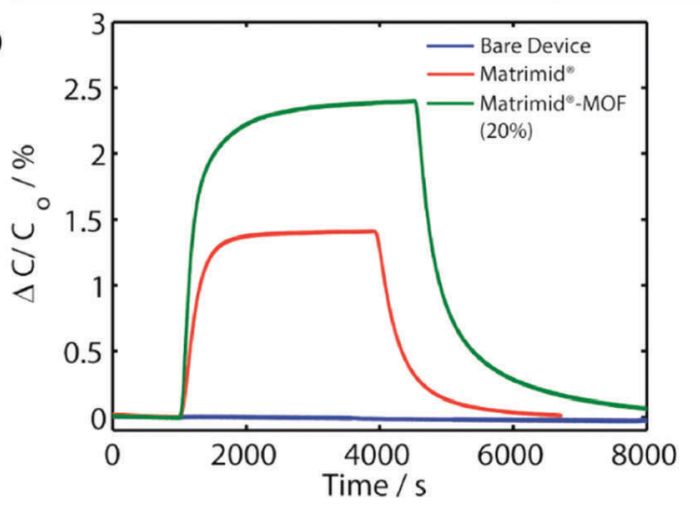

Fig. 26 Miniaturization of chemicapacitors. (a) Overview of the device layout of a CMOS fabricated IDE chemicapacitor. Left: Computer-assisted design: the red rectangle marks the active area. Right: Picture of the actual device after coating with MOFapolymer (Matrimid ${ }^{\mathbb{R}}$ ). Bottom: Schematic cross section of the IDE capacitor. (b) Chemicapacitive response over time of the device and two reference devices upon exposure to $0.5 \%$ methanol followed by desorption. Adapted with permission from ref. 411 . Copyright 2016 American Chemical Society. 
of planar IDEs that were downsized to $0.5 \mu \mathrm{m}$ width and $1 \mu \mathrm{m}$ spacing to optimize the capacitance response. Furthermore, custom lithographic fabrication of the IDE geometry enables matching of the electric field to the MOF layer thickness and thereby optimizing sensitivity, response time and recovery time. Device operation was tested through impedance spectroscopy at room temperature $(20 \mathrm{~Hz}-1 \mathrm{MHz})$. Consistently with the presence of accessible pore space, the MOF functionalized sensor showed a response roughly double that of a sensor coated with polymer alone upon exposure to $0.5 \%$ methanol (Fig. 26b). Pursuing an analogous path of CMOS compatible integration, Eddaoudi and co-workers reported solvothermal growth of Y-FUM sensing films directly on SAM-functionalized IDEs ( $4 \mu \mathrm{m}$ width, $5 \mu \mathrm{m}$ spacing). ${ }^{412}$ This environmentally stable MOF was selected based on its high affinity for $\mathrm{H}_{2} \mathrm{~S}$, for which monitoring in the range of the toxicity exposure limit of $100 \mathrm{ppb}$ is an industrial need. Sensing experiments show a quasi-linear capacitance response over the extraordinarily

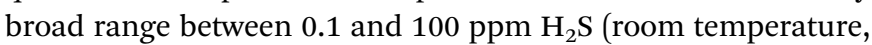
unspecified frequency). The detection limit of the sensor extrapolated from the response between 100 and 1000 ppb was as low as $5.4 \mathrm{ppb}$. Moreover, sensor stability over 12 weeks of testing and high selectivity to $\mathrm{H}_{2} \mathrm{~S}$ over other gases such as $\mathrm{NO}_{2}$, $\mathrm{CH}_{4}, \mathrm{H}_{2}$ and toluene was demonstrated.

Potentiometry and chemitransistors. Potentiometry utilizes the potential difference between two electrodes for chemicalto-electrical transduction and is performed under zero current conditions. Typically a potentiometric sensor consists of a reference electrode that is kept (or presumed) at a constant potential and a chemically sensitive electrode. The work function $(\Phi, \mathrm{eV})$ is defined as the thermodynamic work needed to extract an electron from the Fermi level of a conductor and place it in a vacuum just outside the material. ${ }^{413}$ Physicochemical models for the chemical modulation of the work function of electrodes in the gas and liquid phase have been previously described. ${ }^{414-416}$ For the following discussion, it is important to keep in mind that the work function of an electrode depends to a large degree on interface phenomena, including the surface termination chemistry, the adsorbate phase and the electric field. Intuitively, it can be understood that the presence of dipoles and charge density (in the form of molecular orbitals) near the electrode surface alters the barrier for removal of electrons from the solid and therefore the work function.

A well-established analytical circuit to measure the work function of conductive materials is the Kelvin Probe (KP). In the KP method, a parallel-plate capacitor is constructed consisting of the analytical electrode and a vibrating reference electrode (Fig. 27a). ${ }^{414}$ When contacted through an external circuit, the Fermi levels of the two electrodes will equalize and, as a result of the migration of electrons, the capacitor charges proportionally to the work function difference, i.e. the contact potential difference (CPD, V). The working principle of the KP relies on applying a harmonic vibration to the reference electrode by a piezoelectric actuator. The periodically changing distance between the electrodes causes an oscillation of the capacitance

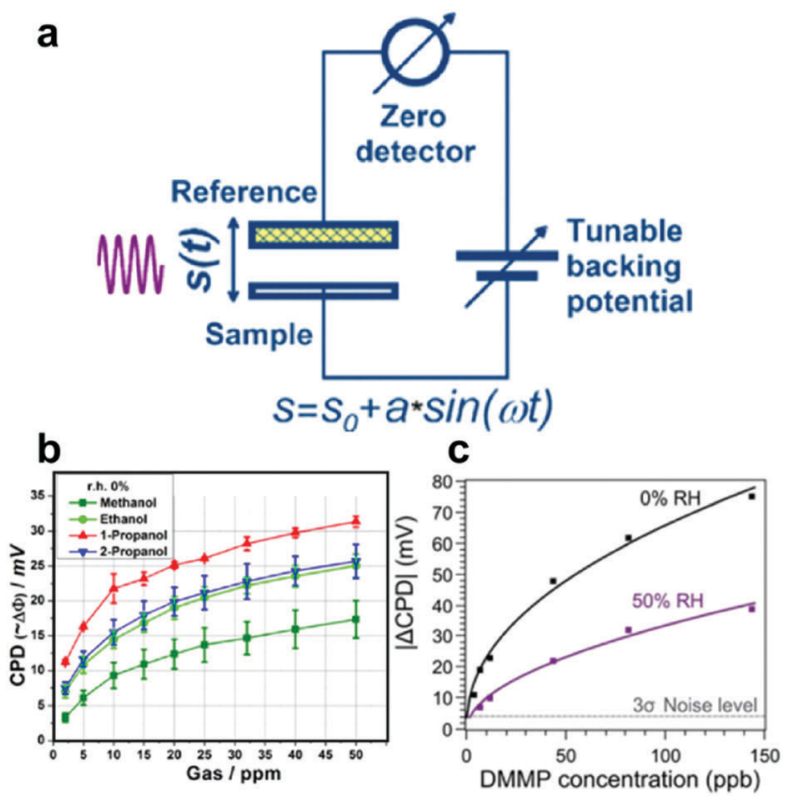

Fig. 27 KP gas sensing by chemical modulation of the work function of the underlying substrate. (a) Layout of KP measurement circuit. Reproduced with permission from ref. 416. (b) CPD of HKUST-1/TiN as a function of the trace ppm concentration of different alcohols. Adapted from ref. 419. Copyright 2014 American Chemical Society. (c) CPD of UiO$66-\mathrm{NH}_{2} / \mathrm{Si}$ as a function of dimethyl methylphosphonate (DMMP) trace ppb concentration in dry and humid conditions. Reproduced from ref. 423.

that can be used to infer the work function of the analytical electrode by taking into account the known reference. Chemical work function modulation can be measured in real time by introducing analytes during KP monitoring of the analytical electrode. The sensing response, $\triangle \mathrm{CPD}$, is interpreted in the assumption that the analyte does not interact with the inert reference electrode. The KP method is well-established as a method to screen the potentiometric sensing performance of (semi)conductive materials based on their surface interactions. However, recent studies demonstrated that analyte-permeable dielectric sensing materials can also be screened using the KP method. ${ }^{416,417}$ As the notions of Fermi level equalization and work function cannot be extended to insulating materials, the KP method in these cases evaluates the influence of the dielectric layer on the potentiometric response of the underlying electrode.

The KP sensing response of dielectric MOF films deposited on (semi)conducting electrodes were investigated in a few studies. Davydovskaya and co-workers utilized HKUST-1 microcrystals drop casted on TiN electrodes to demonstrate CPD responses upon exposure to trace concentrations of different aldehydes. ${ }^{418}$ The reversible responses were particularly significant in case of pentanal (14 $\mathrm{mV}$ at $10 \mathrm{ppm})$, whereas only minor (tenfold lower) responses were observed for longer or shorter chain analogues, such as hexanal or propanal. According to the authors, these results seem consistent with a close match in polarity and size between the analyte and the MOF cages. In any case, the results proved an undisputable correlation between the chemical and structural features of the MOF 
and the chemical modulation of the underlying electrode. In a follow-up study, a range of small alcohols (methanol, ethanol, 1-propanol, propanol) showed similarly high responses at comparable concentrations (10-20 mV at $10 \mathrm{ppm})$, consistent with the anticipated stronger adsorption of these compounds in HKUST-1 (Fig. 27b). ${ }^{419}$ For a better understanding of the results, QCM and KP responses of HKUST-1 films were recorded and compared, in dry conditions as well as at $40 \%$ $\mathrm{RH}$. This comprehensive investigation showed that the interaction with analytes could be significantly influenced by the presence of water in the framework. ${ }^{419,420}$ The unsaturated metal nodes of HKUST-1 served as a preferential strong adsorption sites in dry conditions, whereas the interaction of the analytes with adsorbed water clusters is dominating in the presence of humidity. The same research group also reported exploratory results on using the unsaturated metal nodes in MOF-74 for selective $\mathrm{CO}_{2}$ sensing, ${ }^{421}$ and the complementary use of MOFs and catalytically active electrodes for potentiometric sensing. ${ }^{422}$ Recently, Ameloot and co-workers utilized the KP sensing approach to demonstrate that the high affinity of UiO-66- $\mathrm{NH}_{2}$ for toxic organophosphates can be exploited in sensors (Fig. 27c). ${ }^{423}$ The selection of this particular MOF was inspired by its reported catalytic activity for decomposition of such compounds in buffered media. ${ }^{424,425}$ The MOF powder was drop casted on silicon and the assembly was benchmarked in detection of the nerve agent simulant dimethyl methylphosphonate (DMMP). This study showed a significant and reversible response to DMMP concentrations in the lower ppb range $(24 \mathrm{mV}$ at $10 \mathrm{ppb}$ ) and an extrapolated detection limit $<1 \mathrm{ppb}$ in ideal conditions, which is among the lowest ever reported in KP sensing. Remarkably, at $50 \% \mathrm{RH}$, enough to saturate the nanopores of the MOF with water, DMMP could still be detected down to roughly $2 \mathrm{ppb}$. DFT modeling of the adsorptive interactions between the analyte and MOF, indicated that the combined interaction with the amino linker and the cluster $\mathrm{Zr}-\mathrm{OH} / \mathrm{Zr}-\mathrm{OH}_{2}$ groups is probably responsible for the very high partition coefficient of DMMP. The study demonstrates that judicious matching of the MOF pore space to the analyte can greatly advance sensitivity and selectivity for particularly demanding analytes.

The KP method is typically not utilized in miniaturized devices due to scaling issues for the oscillating electrodes. However, potentiometric effects similar to those registered by the KP method can be utilized in many types of field effect devices, of which chemically sensitive field effect transistors (CHEMFETs) are arguably the most technologically relevant. ${ }^{426,427}$ The operation principle of CHEMFETs is based on chemical work function modulation of the gate electrode (or the channel semiconductor). In a FET device, the threshold voltage $\left(V_{\mathrm{T}}\right)$ is proportional to the difference between the work functions of the gate and channel (9): ${ }^{428}$

$$
V_{\mathrm{T}} \propto \Phi_{\text {gate }}-\Phi_{\text {channel }}
$$

In CHEMFETs, $V_{\mathrm{T}}$ is determined from the $I_{\mathrm{DS}}-V_{\mathrm{G}}$ transfer characteristics and related to the analyte concentration. For instance, in (n-channel) CHEMFETs operated in the saturation regime, the $I_{\mathrm{DS}}$ at a constant gate voltage is related to the square of $V_{\mathrm{T}}$, with $V_{\mathrm{G}}$ and $K_{\mathrm{dev}}$ the externally applied gate voltage and a device constant, respectively (10): ${ }^{428}$

$$
I_{\mathrm{DS}}=K_{\mathrm{dev}}\left(V_{\mathrm{G}}-V_{\mathrm{T}}\right)^{2}
$$

This equation illustrates the direct electrical readout of chemical work function modulation in CHEMFET devices. CHEMFETs can also be considered impedance transformers, as the impedance of the gate-channel capacitor is converted to an associated $I_{\mathrm{DS}}$. As such, the CHEMFET provides options for intrinsic amplification of the signal. ${ }^{429}$ Moreover, a CHEMFET can be designed to provide both work function and impedance information, through simultaneous determination of the $I_{\mathrm{DS}}-V_{\mathrm{G}}$ and $I_{\mathrm{DS}}-V_{\mathrm{DS}}$ characteristics. These characteristics and the option to obtain multidimensional information offer an important advantage over other sensor designs.

Though MOF CHEMFETs are yet to be realized, two recent reports show progress in this direction. Firstly, De Smet and co-workers demonstrated metal-organic polyhedra (MOP) silicon nanowire CHEMFETs (Fig. 28a). ${ }^{430}$ MOPs are discrete molecular cages, often considered zero-dimensional MOF analogues. Similar to MOFs, they contain well-defined cavities and are constructed from tunable metal ions and organic linkers. In this study, CMOS fabricated nanowire FETs (i.e. containing an active channel in the form of a single silicon nanowire) were functionalized by a monolayer of Cu-MOPs selected for binding of 2,4,6-trinitrotoluene (TNT). The $I_{\mathrm{DS}}$ sensitivity towards TNT was raised by three orders of magnitude by the MOP functionalization (in ethanol, down to 100 pM). Secondly, Rao and co-workers reported MOF@conducting-polymer CHEMFETs fabricated by spin coating a mixture of the copolymer diketopyrrolopyrrole thiophene-vinylene-thiophene (DPP-TVT) and the MOF Cd(NDC) ${ }_{0.5}(\mathrm{PCA})$ (NDC = 2,6-napthalenedicarboxylate; $\mathrm{PCA}=4$-pyridinecarboxylate) on pre-fabricated BG-BC devices (Fig. 28b). ${ }^{431}$ Decreasing $I_{\text {DS }}$ was observed at different gate voltages upon exposure to saturated streams of nitroaromatic explosives. This observation pointed to a charge-carrier trap mechanism resulting from the electron-withdrawing nature of the analytes, potentially amplified by accumulation of the analyte in the MOF. Consistent with this hypothesis, a greater effect was observed for analytes with multiple electron withdrawing nitro moieties on the aromatic ring. However, the function of the MOF in the composite is somewhat ambiguous, as reference devices containing only the polymer were not subjected to comparative tests.

Electrochemical sensors and voltammetry. Electrochemical cells consist of two electrodes contacted at one end through an electrical circuit and at the other end through an electrolyte (often complemented by a high ohmic third electrode as a reference at constant potential). When utilized as electroanalytical devices, these cells offer a lot of opportunities for chemical-toelectrical transduction. Voltammetric methods measure current as a response to the waveform of an applied potential. Amperometry designated different types of single-potential voltammetry. However, the terms are often used in an interchangeable way. Electrochemical sensors are distinguished from the previous sensors by the fact that the (Faradaic) current in the measurement circuit is associated with reduction or oxidation of the analyte and 

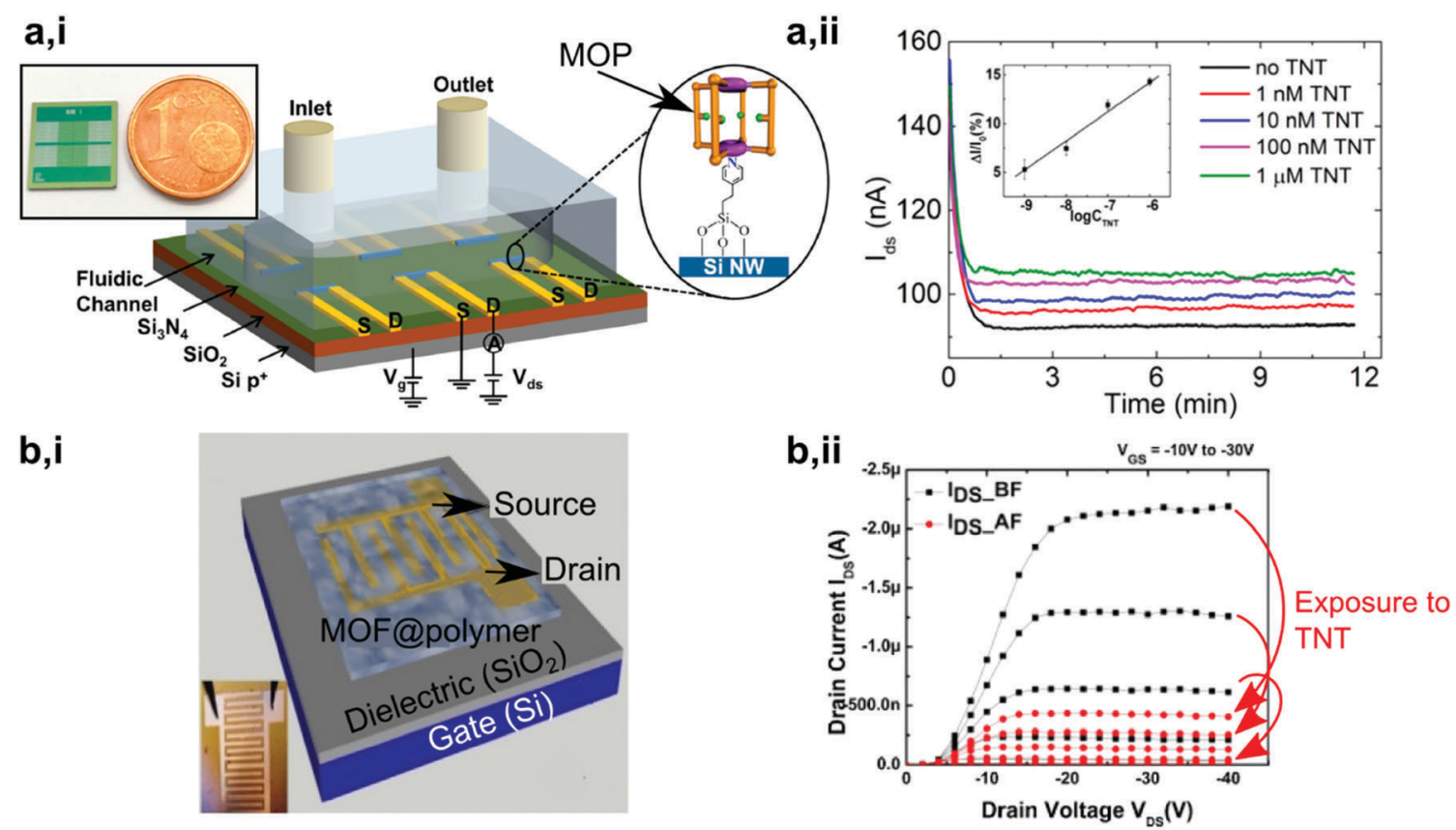

Fig. 28 CHEMFET sensors. (a,i) Array of Cu-MOP functionalized CHEMFETs, schematic layout of device and chip packaging. Inset: Optical picture of the chip. (a,ii) I DS response over time to different concentration of TNT. Adapted with permission from ref. 430. Copyright 2016 American Chemical Society. $(b, i)$ MOF@polymer composite BG-BC CHEMFET layout. Inset: Picture of the applied drain and source contacts during experimentation. (b,ii) $I_{D S}-V_{D S}$ curves at different $V_{\mathrm{G}}$, before (black) and after (red) exposure to TNT. Adapted with permission from ref. 431. Copyright 2016 Elsevier.

is coupled to an opposite ionic current in the electrolyte. Electrochemical voltammetry is the operation principle in various types of microelectrode sensors. ${ }^{432}$ Electrochemical pathways are of particular interest to connect aqueous (bio)chemistry to electronics. A growing number of studies focuses on electrochemistry at the interface between aqueous analytes and MOF functionalized electrodes. Various dielectric MOFs were utilized in composite electrocatalysts, either to immobilize the active sites on an electrode or to enhance the overall activity and selectivity. Analytes of interest in these voltammetric sensing studies were aqueous contaminants (e.g. dihydroxybenzene ${ }^{433}$ hydrazine ${ }^{434,435}$ ), biomolecules or metabolites (e.g. acetaminophen, ${ }^{436}$ ascorbic acid, ${ }^{437,438}$ L-cysteine, ${ }^{439}$ dopamine ${ }^{436-438,440-442}$ chlorogenic acid ${ }^{443}$ glucose ${ }^{444}$ hydrogen peroxide,${ }^{445,446}$ tryptophan, ${ }^{447}$ urea, ${ }^{448}$ uric acid $^{437,438,440}$ ). A particularly elegant example of such routes was demonstrated by Zheng and co-workers: ZIF-8 was coated on one-dimensional arrays of metal oxide semiconductor nanorods to fabricate ZnO@ZIF-8 core-shell nanorod composites. These core-shell composites functioned as photoelectrochemical sensors that utilize the size-selective sieving properties of ZIF-8 to create distinct photoelectrochemical responses upon UV-excitation, depending upon the size of the hole scavenger molecules present in solution. ${ }^{445}$

Although research in MOF sensing is dominated by volatile analytes, arguably inspired by the extensive literature on gas separation and storage, such molecules have not yet been targeted in electrochemical voltammetry. Nevertheless, opportunities may be found in analytes partitioning between the gas and electrolyte phases. Jeong and co-workers demonstrated selective $\mathrm{CO}_{2}$ sensing through electrochemical impedance spectroscopy of a MOF in an electrochemical cell. ${ }^{449}$ A pressed pellet of Rb-CD-MOF saturated with methanol and contacted using silver conductive glue was utilized as electrolyte. Analysis of the impedance spectra $(0.1 \mathrm{~Hz}-30 \mathrm{MHz})$ showed that proton conductivity in the cell gradually dropped 550-fold upon exposure of the framework to different $\mathrm{CO}_{2}$ concentrations. It was hypothesized that the effect was triggered by reversible binding of $\mathrm{CO}_{2}$ as carbonates to the free hydroxyls in framework. Semi-log correlated $\mathrm{CO}_{2}$ sensor responses were demonstrated at room temperature for various concentrations between zero and 100\% (Fig. 29).

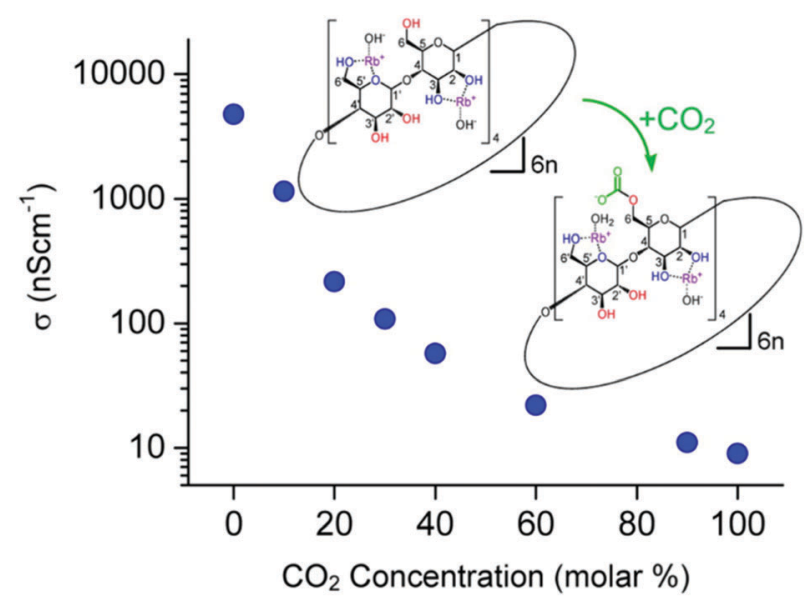

Fig. 29 Implementation of a MOF for electrochemical impedance sensing. The proton conductivity shifts upon carbonate formation were utilized to reversibly quantify atmospheric $\mathrm{CO}_{2}$ over a broad concentration range. Reproduced with permission from ref. 449. Copyright 2014 American Chemical Society. 
Analogous gas sensing routes through Faradaic impedance can by envisioned as a next step. However, this application relies chiefly on the design of MOFs as effective solid electrolytes. Traditionally, the volatile constituents of electrochemical gas sensors are the main bottleneck limiting sensor lifetime, operating temperature range and electrical potential window. ${ }^{432}$

4.2.3 Optoelectronic sensors. Interaction of light with matter involves reflection, scattering, refraction and absorption, potentially followed by emission (i.e. photoluminescence). As previously discussed in Section 2, such interactions are often guestdependent in porous MOFs. Hence, they offer opportunities for chemical sensing via chemical-to-optical and optical-to-electrical transduction pathways in MOF-based optoelectronic devices. Routes for on-chip integration of optical elements such as lasers, waveguides, filters and even full spectrophotometers, are rapidly developing and offer new opportunities for integrated optical sensors. ${ }^{450}$ Many spectroscopic studies have been focused on tailoring of MOF to achieve superior optical responses upon guest uptake. In particular, host-guest responsive photoluminescence has become one of the most active domains in MOF research. The corresponding material design strategies have recently been comprehensively reviewed. ${ }^{119,451}$ The following discussion is limited to recent studies that actively target integration of MOFs in optoelectronic sensors.

A scalable device for optical absorption gas sensing was recently demonstrated by Toda and co-workers. ${ }^{452}$ To fabricate the device, a HKUST-1 coated polytetrafluoroethylene filter was mounted between two optic fibers, one equipped with a blue light source and the other with a photodiode (Fig. 30). This measurement cell was utilized to determine the transmittance

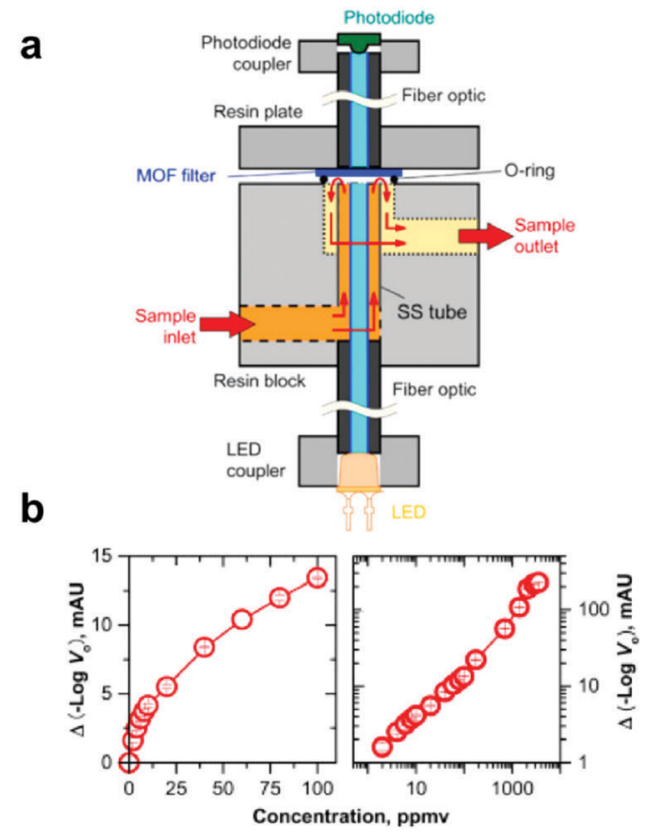

Fig. 30 Transmittance fiber optic device for gas sensing through the colorimetric changes in HKUST-1 upon hydration and dehydration. Reproduced with permission from ref. 452. Copyright 2015 Elsevier. of blue light through the MOF upon exposure to different $\mathrm{RH}$ air streams. The significant color shift of HKUST-1 upon water binding has been previously described in detail. ${ }^{363}$ The device showed a quasi-linear response in trace water sensing, reversibly covering an extraordinary dynamic range of five orders of magnitude (50 ppb-2000 ppm). This device provides an excellent example of how guest-induced spectroscopic shifts can be utilized in chemical-to-optical-to-electrical transduction.

The refractive index shift displayed by MOFs upon guest adsorption offers interesting options for optical sensing as well. The magnitude of this phenomenon was first demonstrated by Férey and co-workers for spin coated MIL-101 thin films on silicon. Refractive index increases up to thirty percent were observed through spectroscopic ellipsometry upon exposure of the film to different volatile molecules. ${ }^{237}$ For practical application of this effect, the refractive index shift needs to be transformed to a measureable optical signal. A range of strategies to achieve this goal relies on mesoscopic structuring of MOFs as photonic etalons, Bragg stacks, two-dimensional, or three-dimensional photonic crystals. As a common feature, these structures consist of regularly repeated regions of high and low refractive index materials. Photonic behavior resulting in observable colors through interference and diffraction occurs when the periodicity is roughly half the wavelength (i.e. 200-400 $\mathrm{nm}$ for the visible range). Readout is typically performed by monitoring color changes that correlate a change in refractive index contrast upon analyte adsorption.

Hupp and co-workers showed that MOF thin films can be exploited as guest-sensitive etalons (or Fabry-Pérot interferometers). ${ }^{177}$ In their study, ZIF-8 thin films of uniform thickness corresponding to optical wavelengths were deposited on glass substrates. The samples showed distinct interference fringes in the visual range as a result of diffraction and interference at the glass-MOF and MOF-air interfaces. Substantial red shifts (up to $49 \mathrm{~nm}$ ) were observed upon exposure of the ZIF-8 thin films to different gases and vapors. In a follow-up study, Bragg stacks consisting of three repeated Pd/ZIF-8 bilayers, were fabricated on glass by ZIF-8 deposition alternated by Pd sputtering. ${ }^{108}$ Adding layers to a Bragg stack leads to increased relative intensity of the interference fringes and therefore improved sensitivity in detecting peak shifts. As a proof-of-concept case, quantitative sensing of hydrogen at concentrations between 0 and 5\% was demonstrated. Lotsch and co-workers, fabricated analogous Bragg stacks by alternatingly spin coating colloidal suspensions of ZIF-8 and $\mathrm{TiO}_{2}$ nanoparticles on silicon (Fig. 31a). ${ }^{238}$ In this example, reflectance spectra fringe peak shifts of up to $100 \mathrm{~nm}$ were shown upon exposure to various alcohols. In a follow-up study, it was demonstrated that the combined responses of Bragg stacks consisting of different MOFs offers options for optical analyte recognition by using MOFs with different polarities, namely ZIF-8, HKUST-1 and CAU-1-NH $\mathrm{NH}_{2}$. Fingerprint analysis of different alcohols was demonstrated by principal component analysis of the visual shifts observed for the different Bragg stacks. ${ }^{453}$ As an additional route for analyte-selectivity, Wang and co-workers demonstrated how guest-specific framework breathing behavior can be exploited to govern the kinetic and equilibrium response to 

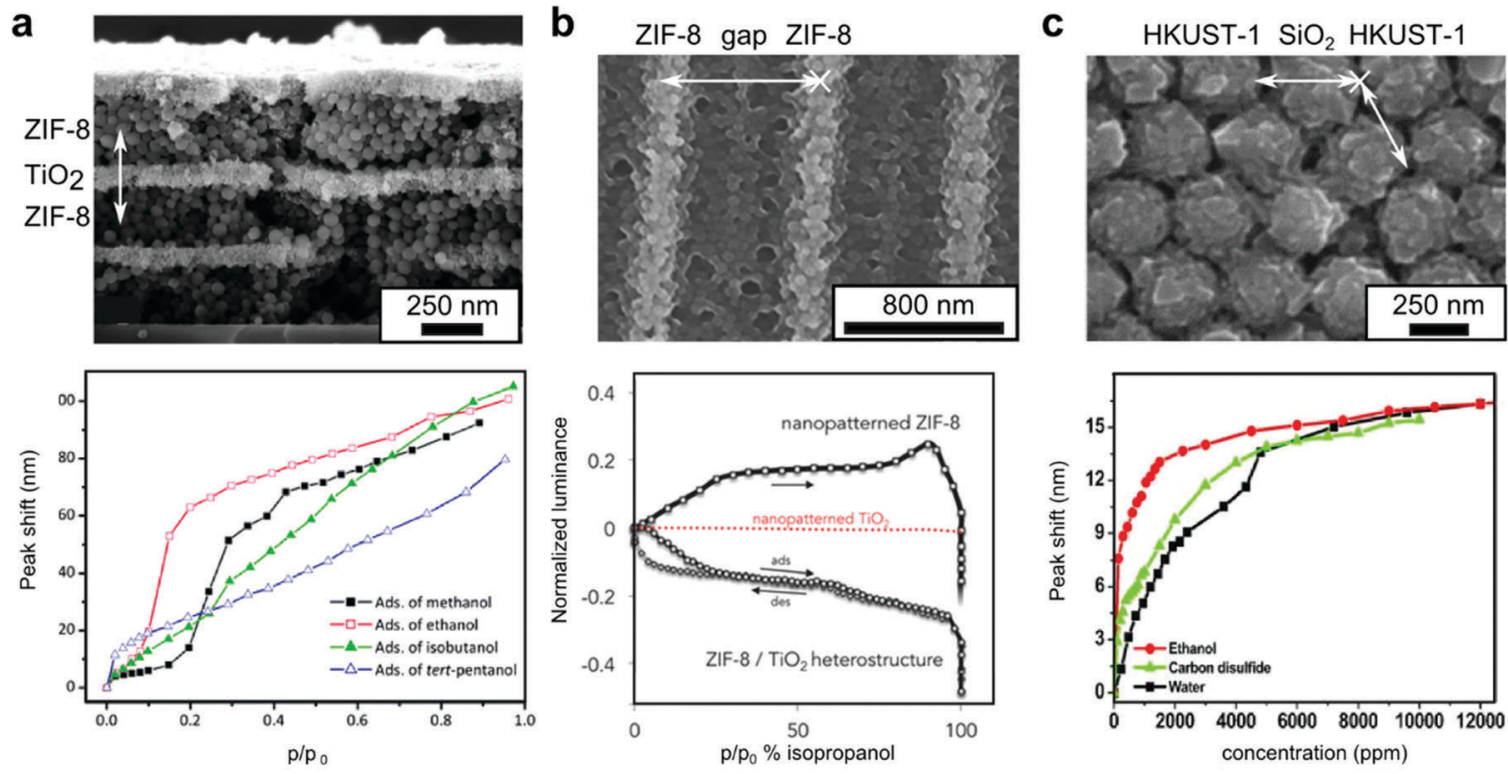

Fig. 31 Photonic films and assemblies for vapor sensing. (a) $\mathrm{ZIF}-8-\mathrm{TiO}_{2}$ one-dimensional photonic structure (Bragg stack). Top: Electron microscopy cross section. Bottom: Reflectance spectral shifts recorded as a function of different organic vapor concentrations. Adapted with permission from ref. 238. (b) ZIF-8 two-dimensional photonic structures. Top: Electron microscopy top view of surface-patterned ZIF-8. Bottom: Reflected luminance recorded by smartphone CCD device at different isopropanol partial pressures. Adapted with permission from ref. 455 . Copyright 2015 Wiley $\&$ Sons. (c) HKUST-1-SiO 2 three-dimensional photonic crystal structure. Reflectance spectral shifts recorded as a function of different analyte vapors. Adapted with permission from ref. 457. Copyright 2011 Wiley \& Sons.

different organics (MIL-88B- $\mathrm{NH}_{2}-\mathrm{TiO}_{2}$ Bragg stack). ${ }^{454}$ Wöll and co-workers fabricated HKUST-1-ITO Bragg stacks by alternated SURMOF LPE spray deposition and ITO sputtering. ${ }^{192}$ In this study, Bragg stack reflectivity of roughly $80 \%$ was observed, which exceeds previous examples. The reduced scattering losses and high optical quality of the thin films grown by LPE could be beneficial for their performance in chemical-to-optical transduction in future applications.

The previous Bragg stack photonic structures are characterized by a periodicity parallel to the substrate. Two-dimensional photonic crystals are equivalent photonic structures with a periodicity perpendicular to the substrate, which is highly versatile as it can be applied by highly-controllable lithographic patterning. Faustini and co-workers fabricated two-dimensional ZIF-8 photonic crystals (ZIF-8-air and ZIF-8-TiO ${ }_{2}$ ) by soft-lithographic nanoimprinting of nanocrystals on different substrates (Fig. 31b). ${ }^{455}$ Using a simple consumer-grade charge-coupled device (CCD) camera, measured reflected light could be correlated to isopropyl alcohol concentrations. Three-dimensional photonic crystals are a combination of the strategies discussed above. $\mathrm{Li}$ and co-workers were the first to demonstrate fabrication of three-dimensional MOF photonic crystals. ${ }^{456}$ HKUST- 1 and ZIF-8 were solvothermally grown in the interstitial space of a close packed film of monodisperse polystyrene beads on glass substrates. After removal of the polymer template, the reflectance spectra of the structure showed a redshift response (up to $75 \mathrm{~nm}$ ) upon exposure to methanol vapor. Secondly, Hupp and co-workers demonstrated MOF-SiO ${ }_{2}$ photonic structures through HKUST-1 deposition in the interstitial space of predeposited $\mathrm{SiO}_{2}$ photonic crystal films (Fig. 31c). The transmittance spectral shifts were utilized for carbon disulfide sensing in the range between 100 and $10000 \mathrm{ppm} .{ }^{457} \mathrm{~A}$ third general strategy, reported by Huo and co-workers, demonstrated layer-by-layer Langmuir-Blodgett coating of self-assembled monolayers of highly monodisperse MOF nanocrystals. ${ }^{458}$ Utilizing the strategy for UiO-66, transmittance spectra red shifts (up to $30 \mathrm{~nm}$ ) upon exposure to different volatile organics was shown for a five layer coating. Recent studies have underlined the potential advantages of mesoscopic design and fine-tuning of three-dimensional MOF photonic crystals. Firstly, two-mode signal transduction based on photonic eigenmode, as well as Fabry-Pérot oscillation was demonstrated for monolayers consisting of MOF hollow spheres. ${ }^{459}$ The performance was further increased by sandwiching the monolayer between highly reflective films. ${ }^{460}$

Surface plasmon resonance (SPR) is an electromagnetic phenomenon that can be utilized to detect extremely small changes in refractive index. Recently, a lot of recent progress has been made in the fabrication of miniaturized SPR biosensors relying on analyte recognition by conjugated antibodies. ${ }^{461}$ MOFs show potential to assist in extending the SPR sensor platform beyond typical biological analytes towards vapors and gases. However, only a few studies that explore the concept of MOF-enhanced SPR have been reported. In a first study, a twodimensional ordered array of silver nanoparticles on glass was coated with HKUST-1 through LPE growth. ${ }^{462}$ The transmittance UV-VIS extinction peak of the sample was monitored spectroscopically upon exposure to pure streams of two analyte gases. It was observed that the peak shift response of the array towards carbon dioxide was enhanced roughly tenfold by the MOF film, whereas no enhancement was seen for non-interacting 
sulfur hexafluoride. In a follow-up study, ITO nanocrystals were sandwiched between a sapphire substrate and the HKUST-1 thin film. ${ }^{463}$ The stacks were used for carbon dioxide sensing based on surface-enhanced infrared absorption (SEIRA), showing a moderate (factor two) increased sensitivity at $0.1 \%$ concentration in comparison to bare substrates. The prototypical HKUST-1 framework is known for its electrophilic open copper sites that can be exploited for high-affinity carbon dioxide binding. Though it is important to note that many MOFs with better performance in selective carbon dioxide capture have been identified in recent research. ${ }^{464}$ A related approach for chemical sensing and recognition of analytes, through their vibrational fingerprint, is surfaceenhanced Raman scattering (SERS). In a proof-of-concept study, a three-dimensional plasmonic array of silver-coated $\mathrm{SiO}_{2}$ nanospheres on glass was coated with ZIF-8 using a stepwise deposition method. ${ }^{465}$ Upon exposure to different vapors, the sample was irradiated with two infrared lasers (excitation and pump wavelengths), and the backscattered light was spectroscopically monitored. Using this method it was shown that traces of vapors such as benzene, toluene, nitrobenzene and 2,6-di-tertbutylpyridine could be identified. By contrast, no signals were observed for the bare substrates in similar conditions. As these molecules are more bulky than the crystallographic pore aperture of ZIF-8, it was hypothesized that adsorption of the analytes on the external surface and grain boundaries of the MOF film is responsible for the enhanced signal. The SPR, SEIRA and SERS proof-of-concepts discussed above show a potential role for MOFs in emerging miniaturized plasmon resonance sensors. Notably, recent studies on bulk MOF and noble metal nanoparticle composites have also demonstrated opportunities for liquid-phase plasmonic chemical sensing, e.g. metal ions, ${ }^{466}$ organic compounds, ${ }^{467}$ and even large macromolecules such as proteins. ${ }^{468}$

\section{Challenges and perspectives: an updated roadmap}

As stated in the introduction, the large and growing interest in MOF materials stems from a desire to better understand and design matter at the atomic scale. At this level, where the boundaries between chemistry and materials science blur, the well-controlled and ordered environment offered by selfassembled crystalline solids enables a range of novel properties. Although in many cases highly promising for application in electronic devices, such work is currently in a proof-ofconcept stage. To further mature and pinpoint actual requirements, the developed concepts need to find their way into other scientific communities, ranging from solid-state physicists to electronic engineers and hardware specialists. As was previously highlighted, the ITRS roadmaps successfully targeted collaborative and highly focused work to solve critical problems inhibiting the advance of microelectronics fabrication. Although MOF research is evidently much less mature, the success of this approach inspired some of us to author the first roadmap for MOFs in electronic devices. ${ }^{5}$ The purpose of the current update is to highlight the significant progress as well as remaining challenges, to stimulate reflection within the community and raise awareness within other fields. In the following we attempt to indicate some of these issues and opportunities, categorized by property, processing step or application.

\section{MOF as electronic conductors}

In the past few years, significant progress has been made in the synthesis of conductive MOFs. These materials offer opportunities for new types of active electronic components. In contrast to purely inorganic or organic conductors such as $\mathrm{MoS}_{2}$ or graphene, which do not easily lend themselves to chemical functionalization, the electronic properties of MOFs can be tuned by chemically altering the linker, metal ion or guest species adsorbed in the pore space. As some elegant first demonstrations have been reported, for instance for layered metal-organic graphene analogues, we expect intense efforts in this area. As experimental results and theoretical calculations provide indications that MOFs might be suitable as semiconductors in future electronics, ${ }^{469}$ the challenge of rational band gap tuning in MOFs is of burgeoning interest. In this context, it will be exciting to see how doping through the inclusion of noninnocent guest molecules in the pores can be used as a design element to generate guest@MOF materials with new properties. Although thus far only pioneering efforts were devoted to this approach (see Section 2.1), ${ }^{14,336}$ the potential offered by porous crystalline (semi)conductors is clear. One can see a conceptual analogy between atomic doping in the crystal lattice of a nonporous semiconductor and molecular doping in the pore space of MOFs. The use of pore space is not limited to electronic conduction; some other uses are described in Section 3.4.

The increasing number of conductive MOFs also highlights the challenge of accurately determining their properties, especially for the more conductive ones. Since MOFs are typically obtained as powders, their properties are often evaluated as pressed pellets. While useful and convenient to gauge bulk behavior, this approach masks the effects of sub-optimal particle-particle and MOF-electrode interfaces. In addition, it fails to indicate anisotropic conduction phenomena. Such data are available from single-crystal measurements, although care should be taken how to attach electrodes. A first step towards the standardization of measurement protocols, which would allow straightforward comparison between different groups, was recently given by an extensive comparison of techniques for a single material. ${ }^{49}$ In this context, the importance of MOFbased FETs must be highlighted as well. In addition to a functional building block of logic circuits, FETs provide direct access to in-depth characterization of their channel material, e.g. in terms of semiconductor type ( $\mathrm{n}$ or $\mathrm{p}$ ), charge carrier mobility and interface defect density. MOF-based diodes are another device type that is useful to study fundamental properties, as well as for practical application as a logic building block. To the best of our knowledge, a MOF-based Schottky diode has been characterized only in one case. ${ }^{51}$ Although the device properties are far short of conventional all-inorganic diodes, they are comparable to those obtained from single-crystal 
organics such as rubrene. ${ }^{470}$ An advantage of the MOF over such organic semiconductors is the higher thermal stability.

\section{MOFs as ionic conductors}

An intensive search for solid electrolytes is ongoing, mainly for energy storage and conversion devices, but as well for electrochemical sensors and ionic transistors. ${ }^{471,472}$ The ordered and porous nature of MOFs offers new opportunities in this area. Most work on ion conducting MOFs has been focused on proton conduction. Nevertheless, conduction of metal ions has been reported, even for divalent ones. In addition to their potential implementation, the well-controlled environment within ion conducting MOFs offers valuable insight into the design parameters of solid electrolytes. For instance, the straightforward introduction of various acidic functional groups is a tremendous tool in clarifying the relationship between proton conductivity, topology, and pore wall functionalization. Such insights will prove valuable to synthesize crystalline MOFs with commercial potential or to implement in amorphous polymer or hybrid electrolytes. ${ }^{473}$

Especially in metal ion conduction for battery applications much room for improvement remains. Although the metal ion conducting MOFs discussed in Section 2.2 are obtained as freeflowing powders, they mostly rely on the inclusion of solventlike molecules in the pores. As these species are small and relatively mobile, the risk for failure through evaporation or diffusion to and reaction at the electrodes is not completely eliminated. Also in this case, rational design through the hostguest chemistry of MOFs can generate entirely solvent-free solid electrolyte materials, for instance through the inclusion of polymer chains from a melt or via in-pore polymerization. ${ }^{44,475}$ Therefore, the MOF porosity itself might not be the crucial characteristic of the resulting electrolyte, but rather the key to designing host-guest interactions in a controlled environment. Compared to polymer-only electrolytes, such host-guest synergy can prevent the formation of less mobile polymer chains (e.g. through crystal domain formation) and, at the same time, align the functional groups on the polymer backbone. ${ }^{476}$ The introduction of ionic liquids to facilitate metal ion conduction has already been demonstrated $;^{88}$ it will be interesting to see whether this approach can be extended to polymerized ionic liquids.

Another approach that perhaps surprisingly has not yet been explored for metal ion conduction is the covalent anchoring of solvent-like moieties to the framework. Again, it should be noted that efficient ion conduction does not require permanent porosity and might in fact benefit from the lack thereof. ${ }^{477}$ This type of solid electrolytes offers perspectives to maintain liquidlike mobility in the pores while the rigid framework provides mechanical stability, thereby overcoming a common trade-off for polymer-only electrolytes. The above approaches could be combined with ionic MOFs. ${ }^{478}$ In an anionic framework employed as solid electrolyte, the immobilized negative charge cannot move in an electric field, resulting in single-ion conductors in which only the metal ion of interest migrates. ${ }^{479}$ If this is not the case, the ionic mobility measured by impedance spectroscopy represents a combined cation and anion mobility.
As for the measurement of electronic conduction, the distinction between pressed-pellet and single-crystal measurements should be made. Isolating the cation mobility in the presence of mobile anions requires further characterization (e.g. ${ }^{7} \mathrm{Li}$ solidstate NMR). On the other hand, deliberately facilitating the migration of anions into battery electrodes is an interesting approach to enhance energy storage capacity. While such insertion reactions are well-established for metal cations (e.g. in Li-ion batteries), it is remarkably challenging to accomplish the same task with negative ions. This difficulty is because electrochemically stable anions are large compared to metal cations; hence, their accommodation requires a much greater accessible volume. Redox-active MOFs with pores large enough to accommodate such anions were already demonstrated as a "dual-ion" cathodes. ${ }^{480}$

\section{Optoelectronics}

Significant progress has been made in exploiting the hybrid organic-inorganic structure of MOFs for energy harvesting. Demonstrations of MOF-based solar cells have mostly been based on the dye-sensitized concept in which photocurrent is generated through charge injection into a semiconductor, typically $\mathrm{TiO}_{2}$. Nevertheless, modeling of the chemical potential of several MOFs identified the misalignment of the LUMO and HOMO levels with the $\mathrm{TiO}_{2}$ conduction band as a possible issue. Interestingly, the richness of MOF chemistry perhaps obviates the need for an additional semiconductor. In such systems, the MOF fulfils the functions of light absorption, carrier generation and transport. For instance, the crystalline organization of porphyrin entities in a $\mathrm{Zn}$ (II) framework resulted in the formation of what may be an indirect bad gap semiconductor with efficient photocarrier generation. We expect concentrated efforts in improving the overall carrier mobilities in such materials, for instance by adding LPE layers to create multi-heteroepitaxial systems. Semiconducting properties can be installed in MOFs through different approaches as well (see Section 2.1). Interestingly, the dyes used in DSSCs are often grafted on the surface of $\mathrm{TiO}_{2}$ particles through carboxylates. As is clear from the success of low-dimensional analogues of inorganic semiconductors in electronically conducting MOFs (e.g. one-dimensional chains), ${ }^{8}$ there is potential to convert the $\mathrm{TiO}_{2}$-dye assembly into an ordered and integrated metal ion-ligand framework. The combination of this approach with the improved properties of light-absorbing linkers when immobilized in a rigid framework or inclusion of different dyes in the pores remains inspiring, for MOFs and related materials. ${ }^{481}$ Taken together, the performance of current prototype devices is encouraging, although far from that of champion devices using all-organic sensitizers. ${ }^{482}$

Besides photovoltaics, other opportunities exist for MOFs in optoelectronics. For example, the photo-induced conductivity response demonstrated by some MOF materials ${ }^{26}$ hints at their potential as detector materials for light and other types of radiation. ${ }^{483}$ Much attention has been devoted to emissiontuning of MOFs for use as down-conversion phosphors. Building on this knowledge and the progress in understanding 
electronic conduction in MOFs, it can be expected that direct emission LEDs with MOFs as active components will be realized in the near future, similar to what has been reported for perovskites. ${ }^{484}$ In addition to fine-tuning light absorption, the regular organization of dyes in the pores of the crystalline solid can create novel guest@MOF emission properties. A fascinating example of such behavior is lasing in single MOF crystals loaded with dye. ${ }^{485,486}$ A similar concept was previously demonstrated in zeolite crystals and pointed out enhanced stability of the occluded dye. ${ }^{487}$ The potential of MOFs, as "metamaterials", for application in non-linear optics was recently reviewed by Vinogradov and co-workers. ${ }^{488}$ Notably, similar to our discussion of electronic applications, the influence of fabrication on the optical performance is rather critical, ${ }^{489}$ but still underexplored.

\section{Dielectrics}

Studies on the use of MOFs as both low- and high- $\kappa$ materials show clear potential but also indicate that a fundamental understanding of the factors contributing to the $\kappa$ and the chemical concepts for their tunability are largely unexplored. From the limited data available, it is clear that to achieve a low- $\kappa$ MOF, ligands with fewer polar functional groups and less-polarized bonds can be introduced. Heavy transition-metal centers should be avoided and guest molecules should be evacuated. As empty space has the lowest possible dielectric constant, increasing the MOF pore size will further decrease the $\kappa$ value. Importantly, very few papers report on the functional testing of integrated MOF dielectrics. As the $\kappa$ value is only one aspect of the suitability of a dielectric for implementation, it will be essential to evaluate other process-specific characteristics. For instance, typical low- $\kappa$ materials have to be hydrophobic, thermally stable up to $350-400{ }^{\circ} \mathrm{C}$, have sufficient mechanical strength, good adhesion to neighboring layers, etc. Outside the typical low- and high- $\kappa$ applications, other potential uses for dielectric MOFs exist. For instance, organic-inorganic dielectric hybrids such as ceramic-polymer composites, are receiving a lot of attention e.g. for application in low-loss dielectric resonators for wireless communication. ${ }^{490,491}$ The potential advantage of MOFs as tunable dielectrics in this context is yet to be explored.

\section{Mechanical properties}

In MOF-based electronic devices, mechanical properties will play a role at the level of both the MOF and the device characteristics. Although mechanical characterization in this context is arguably in the initial stage, the former aspect is considered more "fundamental" and received more attention. Elegant studies demonstrated how gate-opening behavior appears when a rigid MOF is deposited as a very thin film and how such "breathing" is crystal size dependent. ${ }^{366,492}$ While such studies will indirectly aid the integration of MOFs, there is a clear need for further assessment of tuning of engineering-type parameters (e.g. hardness, modulus, stiffness). Although nano-indentation is becoming a more common tool to characterize single MOF crystals and thin film, few materials have been characterized in depth. As already highlighted in Section 4.2, these data will be crucial in interfacing
MOFs with oscillating mass-sensitive devices, for which stiffer materials and tailorability is desirable. Functional testing of MOFs with real substrates under simulated process and operation conditions will rapidly indicate potential mechanical problems, caused for instance by a coefficient of thermal expansion mismatch. The higher expected flexibility of hybrid MOFs compared to inorganic functional materials could be an unexpected advantage. With increasing attention for flexible and wearable devices, elasticity is a highly desired property. This research trend is also reflected in the increasing attention for "molecular layer deposition" (MLD) processes, used in an analogous way to ALD to deposit hybrid coatings. ${ }^{493}$ Accordingly, the MOF community needs to systematically include relevant polymer substrates (e.g. PET) in coating studies, and subject such samples to standardized bending tests. In addition, this approach could open up opportunities to modulate the electronic structure of MOFs through the introduction of mechanical stress and strain. ${ }^{494,495}$ Lastly, recent literature has demonstrated the option to melt and cast some hybrid crystalline frameworks and related glasses. ${ }^{496,497}$ Next to offering new opportunities for structural and mechanical tailoring, these phase transitions can also form a basis for new device fabrication methodologies.

\section{Property modeling}

To design MOF-based electronics, in silico modeling will become an increasingly important tool, in the first place to understand and predict fundamental material properties. In this respect, specific challenges are abound in each of the above sections. For instance, the determination of the band gap and type (direct $v s$. indirect) has important implications for photovoltaics as well as the general use of MOFs as semiconductors. Similarly, modeling will prove valuable in the design of linkers that absorb light in specific parts of the spectrum, in understanding mechanical properties, spin crossover behavior and dielectric constants. In addition, modeling of guest behavior in the MOF pores will not only benefit the design of the best materials for sensor coatings, but guest@MOF approaches as well. Recently, the application of machine-learning algorithms was demonstrated as a tool for elucidation of the (often complex) factors that govern the reaction outcomes in organic-inorganic chemistry. ${ }^{498}$ Basically, the algorithm is optimized to extracts chemical information that is hidden in previous experiments and exploits this data to predict the outcome of related experiments. Since machine-learning tools show an ability to offer insight beyond the ability of human chemical intuition, they could become valuable and widespread screening tools for the MOF community. To access fundamental properties through modeling, the crystalline order of MOFs is an asset as it enables methods using periodic boundary conditions. ${ }^{499}$ Since MOFs have very large unit cells, this approach can reduce the costs of otherwise highly expensive calculations. Some other high-accuracy methods, such as quantum Monte Carlo, are not feasible for systems with such large numbers of electrons. In this regard, MOFs are more challenging than traditional electronic materials, which are typically 
much smaller in terms of unit cell size. Consequently, even though accurate prediction of MOF gas adsorption properties is now feasible, our assessment of the literature indicates that computational methods are at an early stage of development with respect to predicting MOF properties relevant to electronic devices.

Nevertheless, it is a highly positive evolution that modeling data sets and tools are becoming more broadly accessible. Several efforts and initiatives should be mentioned in this context. For instance, a scripted workflow to extract the MOF subset (currently 70000 structures) from the Cambridge Structural Database was recently released by the Cambridge Crystallographic Data Centre. ${ }^{500}$ Previously, the ComputationReady Experimental MOF (CoRE MOF) database was already released by an independent group authors, comprising over 4700 MOF structures from the Cambridge Structural Database for which solvent molecules, partially occupied and disordered atoms were removed. ${ }^{501}$ An even more refined database of over 800 MOFs optimized using periodic density functional theory has also been made publically available. ${ }^{502}$ Similarly, databases and tools such as mofplus.org, ${ }^{503}$ MOFomics, ${ }^{504}$ the Reticular Chemistry Structure Resource, ${ }^{505} \mathrm{Zeo}++,{ }^{506}$ etc. make basic computer-based screening of candidate materials available to non-expert users, for instance to identify frameworks with a specified pore connectivity and windows that would allow passage of certain ions.

\section{Thin films and fabrication}

As is clear from the discussion in the previous sections, reliable methods to deposit uniform and defect-free MOF films will form the basis for microelectronic device fabrication. In addition, such uniform MOF films will in many cases provide a better basis for measurements compared to pressed powder pellets (e.g. $\kappa$ value). Several thin film growth techniques have been further developed as discussed in Section 3.3. Of particular interest in terms of compatibility with other cleanroom processes is the all-vapor phase MOF-CVD route, first illustrated by Ameloot and co-workers for several MOFs based on divalent cations. ${ }^{211}$ Recently, Lausund and Nilsen showed that a related ALD-type CVD process can be used to deposit an amorphous film that upon subsequent exposure to acetic acid vapor resulted in crystalline $\mathrm{Zr}(\mathrm{Iv})$-based UiO-66. ${ }^{507}$ Work by Karppinen and coworkers includes several illustrations of direct MLD growth of crystalline coordination polymers. ${ }^{508-510}$ It will be interesting to see if such all-vapor phase methods can be extended to various MOFs and device applications.

To fabricate the first FETs based on semiconducting MOFs, ${ }^{28,46}$ interfacial growth processes were combined with subsequent film transfer. A recent report has suggested that such approaches could be combined with simultaneous patterning. ${ }^{511}$ Most MOF film growth strategies, including the ones highlighted above, result in non-oriented polycrystalline coatings. In several scenarios, oriented thin films are desired. Currently, the go-to method for oriented MOF films is LPE in combination with a suitable SAM as surface functionalization. However, for applications in which electrical contact with the
MOF is needed, a potentially insulating interstitial layer could be fatal to the device performance. It is therefore interesting that LPE has been demonstrated directly on hydroxylterminated substrates. ${ }^{391}$ Moreover, in some cases the MOF phase has an intrinsic preference for alignment parallel to the surface, because of the formation of well-defined crystal faces or the inherently layered nature of the material. ${ }^{187,208}$ A particularly exciting discovery in this context is the hetero-epitaxial growth of a MOF material on an inorganic substrate. ${ }^{214}$ This report is the first demonstration of both in-plane and out-ofplane orientation of the crystallites in a MOF coating, and therefore suggests the possibility to grow larger oriented crystalline domains. Such single-crystalline domains would be a remarkable achievement with practical implications for device fabrication. For instance, the memristor effect demonstrated for single MOF crystals was not observed for polycrystalline films. It is not unlikely that currently other properties and functions are obscured by sub-optimal thin film deposition methods. In addition, FETs based on single-crystal layers would have minimal structural and electronic defects, therefore allowing a better characterization and understanding of charge transport.

For any film deposition route, several process-related aspects need to be taken into account. Throughput is a parameter mainly discussed in the scale-up of MOF powders, for instance via microwave, sonochemical or mechanochemical methods. Nevertheless, the MOF deposition speed is crucial as well to assess the viability of a film growth process. It is unlikely that film growth processes can build on the approaches developed for powders to decrease growth times. Many MOF deposition processes use large quantities of solvent. Solvents are not only costly, but they present handling, safety, and disposal issues. In the ITRS roadmap, a "green fab" category therefore specifies both process and factory integration requirements that target minimizing both solvent and water usage. Consequently, it is reasonable to expect that future manufacturing with MOFs will require processes that comply with such green chemistry principles. The roughness of the deposited MOF films is another aspect that will require optimization. Compared to established thin film deposition technologies (e.g. sol-gel methods, PVD, CVD, ALD), MOF films typically show a higher roughness, often above $10 \%$ of the film thickness. In the proof-of-concept FET devices, this roughness results in only one side of the selfsupporting film to be smooth enough for device fabrication. While there is a lot of progress to be expected from fine-tuning the crystal growth process, it will be interesting to see whether existing microfabrication approaches such as CMP can address this issue. Patterning via standard lithographic protocols has been scarcely demonstrated for MOFs. It can be challenging to induce isotropic etching for crystalline and porous materials, especially when working with extended single-crystalline domains. Nevertheless, the expected anisotropic etching behavior can become an opportunity when rationalized as in the case of ZIF-8. ${ }^{512}$ In standard silicon-based microfabrication, the introduction of anisotropic etch steps is utilized to generate complex features in a single unit step. Notably, the influence of 
the etching chemistry on important MOF properties such as the specific surface area, pore accessibility and the formation of lattice defects has only scarcely been studied. Similarly, not much is known about the effect of the process steps following MOF deposition or vice versa, the effect of the presence of the MOF during further processing. Although film deposition is considered the first step in the fabrication of microelectronic devices, it is clear that many opportunities remain in this area. Note however that powder processing routes can be acceptable in some "macro"-electronic devices such as batteries and supercapacitor modules. ${ }^{513}$ These types of devices and fabrication processes fall outside the scope of this review.

\section{Chemical sensors}

As is clear from the case study in Section 4.2, chemical sensing is receiving a lot of research attention and might be the first discussed area to shift from the proof-of-concept to the development stage. Arguably, the implementation of MOFs in sensors is the electronic device application that can build the most on the adsorption studies performed for bulk powders. It has been claimed that extending MOFs into the realm of microelectronics is hampered by the paucity of (semi)conducting MOFs. While this is certainly true for applications that require inherent electronic conduction (e.g. active channel in transistors), this is not the case in most sensing schemes, with the notable exception of chemiresistors. A large variety of MOFs could therefore be implemented in future microelectronic chemical sensors. Currently, the main barrier to progress is finding the right combination of MOF material and transduction mechanism for a certain analyte. Also in this case, highthroughput computational screening coupled to a detailed understanding of how host-guest interactions are transduced could be a valuable aid. Nevertheless, it is most probably an oversimplified scenario that single MOFs can be used to selectively detect specific analytes in a complex mixture. Array strategies that interpret the combined output of several sensors functionalized with different MOFs can be a viable solution.
In any case, in a fully integrated MOF sensor, signal processing should be considered. It can be displayed in analog form, subtracted from the reference signal and displayed as a difference, or it can be digitized and processed statistically. Such processing can be carried out on-chip/on-board in "smart sensors" or in a separate processor.

In potentiometric sensing, CHEMFETs functionalized with MOFs to modulate the effective gate voltage are a foreseeable next step. Important for the context of portable and wearable devices, such CHEMFETs are highly scalable and typically operate close to room temperature, resulting in a lower power consumption than typical SMO sensors. In mass-based sensing, QCM and SAW are the most developed acoustic wave sensors. It will be interesting to see the performance gain by combining MOFs with more recent sensors that exploit different elastic wave propagation modes including acoustic-plate-mode and flexural-plate-wave devices. ${ }^{392}$ In voltammetry, the application of MOFs as intrinsically electrocatalytically active electrodes is still uncharted territory. Efforts in this area can be anticipated, as conductive frameworks such as $\mathrm{Ni}_{3}(\mathrm{HITP})_{2}$ can be used as a sole electrode material. ${ }^{513}$ While most sensing-related work focused on gases and vapors, yet again likely inspired by the large related body of work on MOF powders, there are a lot of opportunities in liquid-phase sensing as well, for instance in the detection of heavy metal ions or biomarkers. It is expected that currently unexplored sensor platforms will be included in the range of proof-of-concept MOF-based sensors. Given the wealth of bulk calorimetry data, it is for instance surprising that no MOF-functionalized calorimetric sensors have been reported. In chemical sensors, MOFs are ideally suitable to fulfil other roles than the active sensing layer as well. For instance, a solid electrolyte operating at low temperature and environmentally stable would rapidly find application in electrochemical sensors resistant against electrolyte evaporation. MOFs have already been demonstrated as (selective) preconcentrators that extract a specific analyte or a group of related molecules from a sample for subsequent analysis.

Table 3 SWOT analysis of MOFs in the context of valorization in electronic devices

\begin{tabular}{|c|c|}
\hline Strengths & Weaknesses \\
\hline $\begin{array}{l}\text { - Well-controlled organization at the (sub)nm scale through } \\
\text { self-assembly } \\
\text { - Combined aspects of organic and inorganic materials } \\
\text { - Nanoporosity and rich host-guest chemistry } \\
\text { - Modular design strategies are enabled by crystallinity and } \\
\text { pore space } \\
\text { - Simple organic and inorganic building blocks }\end{array}$ & $\begin{array}{l}\text { - Crystallization needed to achieve distinguishing properties } \\
\text { - Inherent trade-off between properties of typical hard and soft materials } \\
\text { - No perfect examples to illustrate the class of materials as a whole, i.e. } \\
\text { diversity as a source of confusion }\end{array}$ \\
\hline Opportunities & Threats and challenges \\
\hline $\begin{array}{l}\text { - Research in electronics is shifting towards organics and } \\
\text { hybrids } \\
\text { - A clear need for "beyond CMOS" materials and processes } \\
\text { - Broad knowledge platform has been established in other } \\
\text { MOF research } \\
\text { - First commercial breakthroughs will increase interest out- } \\
\text { side the current community }\end{array}$ & $\begin{array}{l}\text { - "Jack-of-all-trades, master of none" perception does not trigger broad } \\
\text { interest (in contrast to e.g. graphene) } \\
\text { - Heuristic material selection and design due to lagging fundamental } \\
\text { understanding } \\
\text { - Lack of focus and standards as a result of the inherent structural and } \\
\text { chemical versatility (no accessible classification for non-experts) } \\
\text { - Limited open dialogue with application-development communities }\end{array}$ \\
\hline
\end{tabular}

- New and better computational tools for property modeling

and screening 
In this scenario, the sensitivity and selectivity requirements for the actual sensor are alleviated and shifted towards the MOF pre-concentrator. $^{226,514,515}$

\section{Valorization perspectives}

Practical applicability should not be the sole guidance of scientific explorations. It is nevertheless interesting to note that the potentially higher added value of MOFs in electronic devices compared to bulk applications alleviates the cost and scale-up restraints for candidate materials. Given the head start of research in gas adsorption, storage and separation, it is natural that the first commercial uses of MOFs focus on niche markets in this field. ${ }^{516,517}$ However, it would not be surprising if future steps in valorization of MOFs would target technology areas that require comparatively very small quantities of material. Examples include the applications highlighted herein, as well as other opportunities in the biomedical field, optical coatings, etc. Any demonstration of commercial viability will lead to increased interest from different domains. As closing in on real-world applications inherently requires an interdisciplinary development effort, any commercial success is likely to benefit the MOF community as a whole through the involvement and interest from different expertise. The challenges discussed in the sections above highlight the need to expand the field beyond the realm of synthetic chemistry and involve those with expertise in solid-state physics, lithography, process design, materials integration and device engineering. As food for thought, we end with our vision on the strengths, weaknesses, opportunities and threats (SWOT) in respect to the valorization of MOFs in electronic devices (Table 3). It is clear that many opportunities remain to bring the fascinating properties of MOFs stemming from their nanoscale organization into real-world applications.

\section{Abbreviations}

\section{General concepts}

$\mathrm{AC}$

ALD

BC-BG

BG-BC

$\mathrm{BHJ}$

CB

CHEMFET

CMOS

CMP

CPD

CTE

CVD

DBA transistor
DC

DFT

DSSC

FET

FRET

GGA

HOMO

IC

IDE

ILD

IRMOF

ITRS

KP

LED

LMCT

LPE

LUMO

MEMS

MIM

MOF

MOF-CVD

MOG

MOP

MLD

PV

PVD

QCM

QD

$\mathrm{RH}$

RMS

SAM

SAW

SEIRA

SERS

SMO

SPR

SURMOF

TG-BC

VB

VOC

Direct current

Density functional theory

Dye-sensitized solar cell

Field effect transistor

Fluorescence resonance energy transfer Generalized gradient approximation

Highest occupied molecular orbital

Integrated circuit; microchip

Interdigitated electrode (device

geometry)

Interlayer dielectric

Isoreticular metal-organic framework

International technology roadmap for semiconductors

Kelvin probe

Light-emitting diode

Ligand-to-metal charge transfer

Liquid-phase epitaxy (MOF growth/ deposition)

Lowest unoccupied molecular orbital

Microelectromechanical systems

Metal-insulator-metal (device geometry)

Metal-organic framework

Metal-organic framework chemical vapor deposition

Metal-organic graphene analogue

Metal-organic polyhedra

Molecular layer deposition

Photovoltaics

Physical vapor deposition

Quartz crystal microbalance (sensor)

Quantum dot

Relative humidity

Root mean square

Self-assembled monolayer

Surface acoustic wave (sensor)

Surface-enhanced infrared absorption

Surface-enhanced Raman scattering

Semiconductor metal oxide (sensor)

Surface plasmon resonance

Surface-anchored metal-organic

framework

Top gate, bottom contact (FET

geometry)

Valence band

Volatile organic compound

\section{Ligands and other molecules}

AZPY

1,3-BDC $\left(\mathrm{H}_{2}\right)$

$\operatorname{BDC}\left(\mathrm{H}_{2}\right)$

$\operatorname{BHT}\left(\mathrm{H}_{6}\right)$

BPAC

$\operatorname{BTB}\left(\mathrm{H}_{3}\right)$

$\mathrm{BTC}\left(\mathrm{H}_{3}\right)$

BODIPY trans-4, $4^{\prime}$-Azopyridine

Benzene-1,3-dicarboxylic acid

Benzene-1,4-dicarboxylic acid

Benzenehexathiol

Bis(4-pyridyl)acetylene

1,3,5-Tris(4-carboxyphenyl)benzene

Benzene-1,3,5-tricarboxylic acid

4,4-Difluoro-4-bora-3a,4a-diaza-s-indacene 
CN

\section{DABCO}

$\operatorname{DCPP}\left(\mathrm{H}_{2}\right)$

\section{DMMP}

\section{NCS}

$\operatorname{NDC}\left(\mathrm{H}_{2}\right)$

$\operatorname{PCA}(\mathrm{H})$

$\operatorname{PDT}\left(\mathrm{H}_{2}\right)$

$\operatorname{TATPT}\left(\mathrm{H}_{6}\right)$

TCNQ

TCPBE $\left(\mathrm{H}_{4}\right)$

TPPE

TMA

TNT

$\operatorname{TTFTB}\left(\mathrm{H}_{4}\right)$

\section{Recurring MOF nomenclature}

$$
\mathrm{Al}_{2}(\mathrm{BDC})_{3}
$$

CAU-1- $\mathrm{NH}_{2}$

CAU-10

$$
\mathrm{Cd}(\mathrm{NDC})_{0.5}(\mathrm{PCA})
$$

Cd-TATPT

$\mathrm{Cu}-(\mathrm{BHT})$

$\mathrm{Co}(\mathrm{IM})_{2}$

$\mathrm{Cu}\left[\mathrm{Ni}(\mathrm{PDT})_{2}\right]$

$\mathrm{Cu}\left[\mathrm{Cu}(\mathrm{PDT})_{2}\right]$

DUT-4

DUT-5

$\mathrm{Fe}_{2}(\mathrm{AZPY})_{4}(\mathrm{NCS})_{4}$

HKUST-1

$\mathrm{In}_{2} \mathrm{BTB}_{3}$

MFU-4

MOF-5

MOF-74
Cyanide anion

1,4-Diazabicyclo[2.2.2] octane

[5,15-Bis(4-carboxyphenyl)-10,20-

diphenylporphyrinato]Zn(II)

Dimethyl methylphosphonate

Thiocyanate anion

Naphthalene-1,4-dicarboxylic acid

4-Pyridinecarboxylic acid

Pyrazine-2,3-dithiol

2,4,6-Tris(2,5-dicarboxylphenylamino)-

1,3,5-triazine

7,7,8,8-Tetracyanoquinodimethane

Tetrakis(4-carboxyphenyl)ethylene

1,1,2,2-Tetrakis(4-(pyridin-4-

yl)phenyl)ethylene

Trimethylamine

2,4,6-Trinitrotoluene

Tetrathiafulvalene tetrabenzoate

MIL-53

MIL-88B

MIL-100

MIL-101

MIL-125

$\mathrm{Ni}_{3}(\mathrm{BHT})_{2}$

$\mathrm{Ni}_{3}(\mathrm{HITP})_{2}$

NU-1000

$\mathrm{Rb}-\mathrm{CD}-\mathrm{MOF}$

$\operatorname{Sr}(1,3-\mathrm{BDC})$

UiO-66

dicarboxylate]

Christian-Albrechts-University of Kiel 1;

$\left[\mathrm{Al}(\mathrm{III})_{4}(\mathrm{OH})_{2}\left(\mathrm{OCH}_{3}\right)_{4}\right][2$-amino-benzene-

1,4-dicarboxylate $]_{3}$

Christian-Albrechts-University of Kiel 10;

$[\mathrm{Al}(\mathrm{III})(\mathrm{OH})][$ 5-R-benzene-1,4-dicarboxylate],

$\mathrm{R}=\mathrm{Br}, \mathrm{NO}_{2}, \mathrm{NH}_{2}$, etc.

$[\mathrm{Cd}(\mathrm{II})]_{2}[$ naphthalene-1,4-dicarboxylate]-

[4-pyridinecarboxylate $]_{2}$

$\left[\mathrm{Cd}(\mathrm{II})_{2} \mathrm{Cl}\right]_{3}[\text { dimethylammonium }]_{15}[2,4,6-$

tris(2,5-dicarboxylatephenylamino)-

1,3,5-triazine $]_{4}$

$[\mathrm{Cu}(\mathrm{II})]_{3}[$ benzenehexathiolate $]$

$[\mathrm{Co}(\mathrm{II})][\text { imidazolate }]_{2}$

[Cu(II)][Ni(II)bis(pyrazine-2,3-dithiolate)]

$[\mathrm{Cu}(\mathrm{II})][\mathrm{Cu}(\mathrm{II})$ bis(pyrazine-2,3-dithiolate)]

TU Dresden 4; $[\mathrm{Al}(\mathrm{III})(\mathrm{OH})][$ naphthalene2,6-dicarboxylate]

TU Dresden 5; [Al(III)(OH)][biphenyl-4,4dicarboxylate]

$[\mathrm{Fe}(\mathrm{II})]_{2}\left[\right.$ trans $-4,4^{\prime}-$

azopyridine $]_{4}[\text { thiocyanate }]_{4}$

ZIF-8

Hong Kong University of Science and Technology 1; $[\mathrm{Cu}(\mathrm{II})]_{3}[$ benzene-1,3,5tricarboxylate $]_{2}$

$[\mathrm{In}(\mathrm{III})]_{3}[1,3,5$-tris(4-carboxylatephenyl)benzene $][1,3,5$-tris $(4$ -

carboxyphenyl)benzene] [oxalate $]_{3}$

Metal-organic framework Ulm University 4 ; $\left[\mathrm{Zn}(\mathrm{II}) \mathrm{Cl}_{4}\right]_{5}[1 H, 5 H$-benzo(1,2-d:4,5d)bistriazolate $]_{3}$

Metal-organic framework 5 ; $\left[\mathrm{Zn}(\mathrm{II})_{4} \mathrm{O}\right]-$ [benzene-1,4-dicarboxylate $]_{3}$

Metal-organic framework 74; $[\mathrm{M}(\mathrm{II})]_{2}[2,5-$ dioxido-1,4-benzenedicarboxylate]

UiO-67

Y-FUM

ZIF

ZIF-4

ZIF-7

ZIF-9

ZIF-14

ZIF-67

ZIF-69

ZIF-90
Materials Institute of Lavoisier 53; [M(III)OH]-

[benzene-1,4-dicarboxylate]

Materials Institute of Lavoisier 88B;

$\left[\mathrm{M}(\mathrm{III})_{3} \mathrm{XO}\right][\text { benzene-1,4-dicarboxylate }]_{3}$, $\mathrm{X}=\mathrm{Cl}^{-}, \mathrm{F}^{-}$, etc.

Materials Institute of Lavoisier 100;

$\left[\mathrm{M}(\mathrm{III})_{3} \mathrm{XO}\right][\text { benzene-1,3,5-tricarboxylate }]_{2}$, $\mathrm{X}=\mathrm{F}^{-}, \mathrm{OH}^{-}$, etc.

Materials Institute of Lavoisier 101;

$\left[\mathrm{M}(\mathrm{III})_{3} \mathrm{FO}\right][\text { benzene-1,4-dicarboxylate }]_{3}$

Materials Institute of Lavoisier 125;

$\left[\mathrm{Ti}(\mathrm{Iv})_{8} \mathrm{O}_{8}(\mathrm{OH})_{4}\right][\text { benzene-1,4-dicarboxylate }]_{6}$

$[\mathrm{Ni}(\mathrm{II})]_{3}[\text { benzenehexathiolate }]_{2}$

$[\mathrm{Ni}(\mathrm{II})]_{3}[2,3,6,7,10,11$-hexaaminotri-

phenylenesemiquinonate $]_{2}$

Northwestern University 1000; $\left[\mathrm{Zr}_{6}\left(\mu_{3}-\right.\right.$

$\left.\mathrm{O})_{4}\left(\mu_{3}-\mathrm{OH}\right)_{4}(\mathrm{OH})_{4}\right][1,3,6,8$-tetrakis $(p$ -

benzoate)pyrene $]_{2}$

$[\mathrm{Rb}(\mathrm{I}) \mathrm{OH}]_{2}[\gamma$-cyclodextrin $]$

$[\mathrm{Sr}(\mathrm{II})]_{2}[\text { benzene-1,3-dicarboxylate }]_{2}$

Universitetet i Oslo (University of Oslo) 66;

$\left[\mathrm{Zr}(\mathrm{Iv})_{6} \mathrm{O}_{4}(\mathrm{OH})_{4}\right][$ benzene-1,4-

dicarboxylate $]_{6}$

Universitetet i Oslo (University of Oslo) 67;

$\left[\mathrm{Zr}(\mathrm{Iv})_{6} \mathrm{O}_{4}(\mathrm{OH})_{4}\right][$ biphenyl-4,4-

dicarboxylate $]_{6}$

$\left[\mathrm{Y}(\mathrm{III})_{6}\left(\mu_{3}-\mathrm{OH}\right)_{8}\right][\text { fumarate }]_{6}$

Zeolitic imidazolate framework

Zeolitic imidazolate framework 4;

$[\mathrm{Zn}(\mathrm{II})][\text { imidazolate }]_{2}$

Zeolitic imidazolate framework 7;

$[\mathrm{Zn}(\mathrm{II})][\text { benzimidazolate }]_{2}$

Zeolitic imidazolate framework 9;

$[\mathrm{Co}(\mathrm{II})][\text { benzimidazolate }]_{2}$

Zeolitic imidazolate framework 14;

$[\mathrm{Zn}(\mathrm{II})][\text { 2-ethylimidazolate }]_{2}$

Zeolitic imidazolate framework 67;

$[\mathrm{Co}(\mathrm{II})][\text { 2-methylimidazolate }]_{2}$

Zeolitic imidazolate framework 69;

[Zn(II)][5-chlorobenzimidazolate $][2-$

nitroimidazolate]

Zeolitic imidazolate framework 8; $[\mathrm{Zn}(\mathrm{II})][\text { 2-methylimidazolate }]_{2}$

Zeolitic imidazolate framework 90;

$[\mathrm{Zn}(\mathrm{II})][$ imidazolate-2-carboxy-

aldehyde $]_{2}$

$\mathrm{Zn}_{6}(\mathrm{BTC})_{4}(\mathrm{TPPE})_{2} \quad[\mathrm{Zn}(\mathrm{II})]_{6}[\text { benzene-1,3,5-tricarboxylate }]_{4^{-}}$

[1,1,2,2-tetrakis(4-(pyridin-4-

yl)phenyl)ethylene]

Zn-DCPP SURMOF-2 [Zn(II)][\{5,15-bis(4-carboxy-phenyl)-

10,20-diphenylporphyrinato\}Zn(II)]

$[\mathrm{Zn}(\mathrm{II})]_{2}[$ tetrakis(4-carboxylatephenyl)ethylene]

$[\mathrm{Zn}(\mathrm{II})]_{2}[$ naphthalene-1,4-dicarboxy-

late $]_{2}[1,4$-diazabicyclo[2.2.2]octane] 


\section{Other materials}

DPP-TVT

FTO

ITO

PB

PEDOT

PET
Diketopyrrolopyrrole thiophenevinylene-thiophene copolymer

Fluorine doped tin oxide

Indium tin oxide

Prussian Blue; Fe(II,III)hexacyanoferrate(II,III)

Poly(3,4-ethylenedioxythiophene)

Polyethylene terephthalate

\section{Acknowledgements}

I. S. acknowledges the Research Foundation - Flanders (FWO) for a postdoctoral fellowship. R. A. acknowledges KU Leuven (STG/14/07BF), FWO (G083016N) and the European Research Council (ERC, VAPORE 716472) for funding. N. C. B. acknowledges the support of the Sandia National Laboratories Truman Fellowship Program. M. A., A. T. and N. C. B. acknowledge the Sandia National Laboratory Directed Research and Development (LDRD) Program. Sandia National Laboratories is a multiprogram laboratory managed and operated by Sandia Corporation, a wholly owned subsidiary of Lockheed Martin Corporation, for the US Department of Energy's National Nuclear Security Administration under Contract DE-AC04-94AL85000.

\section{References}

1 H. Furukawa, K. E. Cordova, M. O'Keeffe and O. M. Yaghi, Science, 2013, 341, 1230444.

2 S. Kitagawa, R. Kitaura and S. Noro, Angew. Chem., Int. Ed., 2004, 43, 2334-2375.

3 G. Férey, Chem. Soc. Rev., 2008, 37, 191-214.

4 B. Li, H.-M. Wen, Y. Cui, W. Zhou, G. Qian and B. Chen, Adv. Mater., 2016, 28, 8819-8860.

5 M. D. Allendorf, A. Schwartzberg, V. Stavila and A. A. Talin, Chem. - Eur. J., 2011, 17, 11372-11388.

6 S. R. Batten, N. R. Champness, X.-M. Chen, J. GarciaMartinez, S. Kitagawa, L. Öhrström, M. O’Keeffe, M. Paik Suh and J. Reedijk, Pure Appl. Chem., 2013, 85, 1715-1724.

7 A. C. Ferrari, F. Bonaccorso, V. Fal'ko, K. S. Novoselov, S. Roche, P. Bøggild, S. Borini, F. H. L. Koppens, V. Palermo, N. Pugno, J. A. Garrido, R. Sordan, A. Bianco, L. Ballerini, M. Prato, E. Lidorikis, J. Kivioja, C. Marinelli, T. Ryhänen, A. Morpurgo, J. N. Coleman, V. Nicolosi, L. Colombo, A. Fert, M. Garcia-Hernandez, A. Bachtold, G. F. Schneider, F. Guinea, C. Dekker, M. Barbone, Z. Sun, C. Galiotis, A. N. Grigorenko, G. Konstantatos, A. Kis, M. Katsnelson, L. Vandersypen, A. Loiseau, V. Morandi, D. Neumaier, E. Treossi, V. Pellegrini, M. Polini, A. Tredicucci, G. M. Williams, B. Hee Hong, J.-H. Ahn, J. Min Kim, H. Zirath, B. J. van Wees, H. van der Zant, L. Occhipinti, A. Di Matteo, I. A. Kinloch, T. Seyller, E. Quesnel, X. Feng, K. Teo, N. Rupesinghe, P. Hakonen, S. R. T. Neil, Q. Tannock, T. Löfwander and J. Kinaret, Nanoscale, 2015, 7, 4598-4810.
Ed., 2016, 55, 3566-3579.

9 R. E. Hummel, Electronic Properties of Materials, Springer New York, New York, NY, 2011.

10 C. H. Hendon, D. Tiana and A. Walsh, Phys. Chem. Chem. Phys., 2012, 14, 13120.

11 Y. Zhou and S. Ramanathan, Proc. IEEE, 2015, 103, 1289-1310.

12 Y. Kobayashi, B. Jacobs, M. D. Allendorf and J. R. Long, Chem. Mater., 2010, 22, 4120-4122.

13 D. M. Pajerowski, T. Watanabe, T. Yamamoto and Y. Einaga, Phys. Rev. B: Condens. Matter Mater. Phys., 2011, 83, 153202.

14 M. E. Foster, K. Sohlberg, C. D. Spataru and M. D. Allendorf, J. Phys. Chem. C, 2016, 120, 15001-15008.

15 L. E. Darago, M. L. Aubrey, C. J. Yu, M. I. Gonzalez and J. R. Long, J. Am. Chem. Soc., 2015, 137, 15703-15711.

16 L. Luo, S. H. Choi and C. D. Frisbie, Chem. Mater., 2011, 23, 631-645.

17 S. Han, S. C. Warren, S. M. Yoon, C. D. Malliakas, X. Hou, Y. Wei, M. G. Kanatzidis and B. A. Grzybowski, J. Am. Chem. Soc., 2015, 137, 8169-8175.

18 A. A. Talin, A. Centrone, A. C. Ford, M. E. Foster, V. Stavila, P. Haney, R. A. Kinney, V. Szalai, F. El Gabaly, H. P. Yoon, F. Leonard and M. D. Allendorf, Science, 2014, 343, 66-69.

19 M. D. Newton, Chem. Rev., 1991, 91, 767-792.

20 B. S. Brunschwig, C. Creutz and N. Sutin, Chem. Soc. Rev., 2002, 31, 168-184.

21 M. D. Allendorf, M. E. Foster, F. Leonard, V. Stavila, P. L. Feng, F. P. Doty, K. Leong, E. Y. Ma, S. R. Johnston and A. A. Talin, J. Phys. Chem. Lett., 2015, 6, 1182-1195.

22 T. C. Narayan, T. Miyakai, S. Seki and M. Dincă, J. Am. Chem. Soc., 2012, 134, 12932-12935.

23 S. S. Park, E. R. Hontz, L. Sun, C. H. Hendon, A. Walsh, T. Van Voorhis and M. Dinca, J. Am. Chem. Soc., 2015, 137, 1774-1777.

24 D. S. Chen, H. Z. Xing, Z. M. Su and C. G. Wang, Chem. Commun., 2016, 52, 2019-2022.

25 P. A. Cox, in Physics and Chemistry of Low-Dimensional Inorganic Conductors, ed. C. Schlenker, J. Dumas, M. Greenblatt and S. van Smaalen, Springer US, Boston, MA, 1996, vol. 354, pp. 255-270.

26 L. Sun, S. S. Park, D. Sheberla and M. Dincă, J. Am. Chem. Soc., 2016, 138, 14772-14782.

27 K. J. Erickson, F. Léonard, V. Stavila, M. E. Foster, C. D. Spataru, R. E. Jones, B. M. Foley, P. E. Hopkins, M. D. Allendorf and A. A. Talin, Adv. Mater., 2015, 27, 3453-3459.

28 G. Wu, J. Huang, Y. Zang, J. He and G. Xu, J. Am. Chem. Soc., 2017, 139, 1360-1363.

29 S. Holliday, J. E. Donaghey and I. McCulloch, Chem. Mater., 2014, 26, 647-663.

30 S. Takaishi, M. Hosoda, T. Kajiwara, H. Miyasaka, M. Yamashita, Y. Nakanishi, Y. Kitagawa, K. Yamaguchi, A. Kobayashi and H. Kitagawa, Inorg. Chem., 2009, 48, 9048-9050. 
31 D. Sheberla, L. Sun, M. A. Blood-Forsythe, S. Er, C. R. Wade, C. K. Brozek, A. Aspuru-Guzik and M. Dincă, J. Am. Chem. Soc., 2014, 136, 8859-8862.

32 M. Usman, S. Mendiratta, S. Batjargal, G. Haider, M. Hayashi, N. R. Gade, J.-W. Chen, Y.-F. Chen and K.-L. Lu, ACS Appl. Mater. Interfaces, 2015, 7, 22767-22774.

33 T. Neumann, J. X. Liu, T. Wachter, P. Friederich, F. Symalla, A. Welle, V. Mugnaini, V. Meded, M. Zharnikov, C. Woll and W. Wenzel, ACS Nano, 2016, 10, 7085-7093.

34 A. Sengupta, S. Datta, C. L. Su, T. S. Herng, J. Ding, J. J. Vittal and K. P. Loh, ACS Appl. Mater. Interfaces, 2016, 8, 16154-16159.

35 J. X. Liu, T. Wachter, A. Irmler, P. G. Weidler, H. Gliemann, F. Pauly, V. Mugnaini, M. Zharnikov and C. Woll, ACS Appl. Mater. Interfaces, 2015, 7, 9824-9830.

36 J. N. Behera, D. M. D’Alessandro, N. Soheilnia and J. R. Long, Chem. Mater., 2009, 21, 1922-1926.

37 J. J. M. Amoore, S. M. Neville, B. Moubaraki, S. S. Iremonger, K. S. Murray, J. F. Letard and C. J. Kepert, Chem. - Eur. J., 2010, 16, 1973.

38 M. Kurmoo, H. Kumagai, K. W. Chapman and C. J. Kepert, Chem. Commun., 2005, 3012-3014.

39 L. Pan, G. Liu, H. Li, S. Meng, L. Han, J. Shang, B. Chen, A. E. Platero-Prats, W. Lu, X. Zou and R.-W. Li, J. Am. Chem. Soc., 2014, 136, 17477-17483.

40 M. D. Allendorf, R. Medishetty and R. A. Fischer, MRS Bull., 2016, 41, 865-869.

41 G. Wegner, Angew. Chem., Int. Ed., 1981, 20, 361-381.

42 C. K. Chiang, C. R. Fincher, Y. W. Park, A. J. Heeger, H. Shirakawa, E. J. Louis, S. C. Gau and A. G. Macdiarmid, Phys. Rev. Lett., 1977, 39, 1098-1101.

43 R. O. Grisdale, J. Appl. Phys., 1953, 24, 1288-1296.

44 J. Roncali, Chem. Rev., 1992, 92, 711-738.

45 E. G. Bittle, J. I. Basham, T. N. Jackson, O. D. Jurchescu and D. J. Gundlach, Nat. Commun., 2016, 7, 10908.

46 X. Huang, P. Sheng, Z. Tu, F. Zhang, J. Wang, H. Geng, Y. Zou, C. Di, Y. Yi, Y. Sun, W. Xu and D. Zhu, Nat. Commun., 2015, 6, 7408.

47 T. Kambe, R. Sakamoto, K. Hoshiko, K. Takada, M. Miyachi, J.-H. Ryu, S. Sasaki, J. Kim, K. Nakazato, M. Takata and H. Nishihara, J. Am. Chem. Soc., 2013, 135, 2462-2465.

48 T. Kambe, R. Sakamoto, T. Kusamoto, T. Pal, N. Fukui, K. Hoshiko, T. Shimojima, Z. Wang, T. Hirahara, K. Ishizaka, S. Hasegawa, F. Liu and H. Nishihara, J. Am. Chem. Soc., 2014, 136, 14357-14360.

49 L. Sun, C. H. Hendon, M. A. Minier, A. Walsh and M. Dinca, J. Am. Chem. Soc., 2015, 137, 6164-6167.

50 L. Sun, T. Miyakai, S. Seki and M. Dinca, J. Am. Chem. Soc., 2013, 135, 8185-8188.

51 B. Bhattacharya, A. Layek, M. M. Alam, D. K. Maity, S. Chakrabarti, P. P. Ray and D. Ghoshal, Chem. Commun., 2014, 50, 7858-7861.

52 J. Liu, W. Zhou, J. Liu, I. Howard, G. Kilibarda, S. Schlabach, D. Coupry, M. Addicoat, S. Yoneda,
Y. Tsutsui, T. Sakurai, S. Seki, Z. Wang, P. Lindemann, E. Redel, T. Heine and C. Wöll, Angew. Chem., Int. Ed., 2015, 54, 7441-7445.

53 Z.-G. Gu, L. Heinke, C. Woell, T. Neumann, W. Wenzel, Q. Li, K. Fink, O. D. Gordan and D. R. T. Zahn, Appl. Phys. Lett., 2015, 107, 183301.

54 P. K. Ross, M. D. Allendorf and E. I. Solomon, J. Am. Chem. Soc., 1989, 111, 4009-4021.

55 F. Tuczek and E. I. Solomon, Coord. Chem. Rev., 2001, 219, 1075-1112.

56 K. T. Butler, C. H. Hendon and A. Walsh, J. Am. Chem. Soc., 2014, 136, 2703-2706.

57 J. A. Botas, G. Calleja, M. Sanchez-Sanchez and M. G. Orcajo, Int. J. Hydrogen Energy, 2011, 36, 10834-10844.

58 J. H. Choi, H. J. Jeon, K. M. Choi and J. K. Kang, J. Mater. Chem., 2012, 22, 10144-10147.

59 H. Q. Pham, T. Mai, N. N. Pham-Tran, Y. Kawazoe, H. Mizuseki and D. Nguyen-Manh, J. Phys. Chem. C, 2014, 118, 4567-4577.

60 L. M. Yang, G. Y. Fang, J. Ma, E. Ganz and S. S. Han, Cryst. Growth Des., 2014, 14, 2532-2541.

61 L. M. Yang, P. Ravindran, P. Vajeeston and M. Tilset, Phys. Chem. Chem. Phys., 2012, 14, 4713-4723.

62 T. Musho and N. D. Wu, Phys. Chem. Chem. Phys., 2015, 17, 26160-26165.

63 A. S. Yasin, J. T. Li, N. Q. Wu and T. Musho, Phys. Chem. Chem. Phys., 2016, 18, 12748-12754.

64 P. Sippel, D. Denysenko, A. Loidl, P. Lunkenheimer, G. Sastre and D. Volkmer, Adv. Funct. Mater., 2014, 24, 3885-3896.

65 J. Liu, W. Zhou, J. Liu, Y. Fujimori, T. Higashino, H. Imahori, X. Jiang, J. Zhao, T. Sakurai, Y. Hattori, W. Matsuda, S. Seki, S. K. Garlapati, S. Dasgupta, E. Redel, L. Sun and C. Wöll, J. Mater. Chem. A, 2016, 4, 12739-12747.

66 X. J. Wang, G. Y. Gou, D. W. Wang, H. Y. Xiao, Y. Liu, M. Zhang, B. Dkhil, X. B. Ren and X. J. Lou, RSC Adv., 2016, 6, 48779-48787.

67 C. H. Hendon and A. Walsh, Chem. Sci., 2015, 6, 3674-3683.

68 S. Chen, J. Dai and X. C. Zeng, Phys. Chem. Chem. Phys., 2015, 17, 5954-5958.

69 B. Zhao, Phys. Rev. B: Condens. Matter Mater. Phys., 2014, 90, 201403(R).

70 L. Dong, Y. Kim, D. Er, A. M. Rappe and V. B. Shenoy, Phys. Rev. Lett., 2016, 116, 096601.

71 M. G. Campbell, D. Sheberla, S. F. Liu, T. M. Swager and M. Dincă, Angew. Chem., Int. Ed., 2015, 54, 4349-4352.

72 T. Rodenas, I. Luz, G. Prieto, B. Seoane, H. Miro, A. Corma, F. Kapteijn, F. Xamena and J. Gascon, Nat. Mater., 2015, 14, 48-55.

73 M. C. So, S. Jin, H. J. Son, G. P. Wiederrecht, O. K. Farha and J. T. Hupp, J. Am. Chem. Soc., 2013, 135, 15698-15701.

74 D. Van Gough, T. N. Lambert, D. R. Wheeler, M. A. Rodriguez, M. T. Brumbach, M. D. Allendorf and E. D. Spoerke, ACS Appl. Mater. Interfaces, 2014, 6, 1509-1514.

75 R. Makiura and O. Konovalov, Sci. Rep., 2013, 3, 2506. 
76 E. Quartarone and P. Mustarelli, Chem. Soc. Rev., 2011, 40, 2525.

77 M. Yoon, K. Suh, S. Natarajan and K. Kim, Angew. Chem., Int. Ed., 2013, 52, 2688-2700.

78 G. K. H. Shimizu, J. M. Taylor and S. Kim, Science, 2013, 341, 354-355.

79 S. Horike, D. Umeyama and S. Kitagawa, Acc. Chem. Res., 2013, 46, 2376-2384.

80 M. Sadakiyo, T. Yamada and H. Kitagawa, ChemPlusChem, 2016, 81, 691-701.

81 S. Bureekaew, S. Horike, M. Higuchi, M. Mizuno, T. Kawamura, D. Tanaka, N. Yanai and S. Kitagawa, Nat. Mater., 2009, 8, 831-836.

82 J. A. Hurd, R. Vaidhyanathan, V. Thangadurai, C. I. Ratcliffe, I. L. Moudrakovski and G. K. H. Shimizu, Nat. Chem., 2009, 1, 705-710.

83 D. Umeyama, S. Horike, M. Inukai, Y. Hijikata and S. Kitagawa, Angew. Chem., Int. Ed., 2011, 50, 11706-11709.

84 D. Umeyama, S. Horike, M. Inukai, T. Itakura and S. Kitagawa, J. Am. Chem. Soc., 2012, 134, 12780-12785.

85 S. Horike, D. Umeyama, M. Inukai, T. Itakura and S. Kitagawa, J. Am. Chem. Soc., 2012, 134, 7612-7615.

86 B. M. Wiers, M.-L. Foo, N. P. Balsara and J. R. Long, J. Am. Chem. Soc., 2011, 133, 14522-14525.

87 R. Ameloot, M. Aubrey, B. M. Wiers, A. P. GómoraFigueroa, S. N. Patel, N. P. Balsara and J. R. Long, Chemistry, 2013, 19, 5533-5536.

88 K. Fujie, R. Ikeda, K. Otsubo, T. Yamada and H. Kitagawa, Chem. Mater., 2015, 27, 7355-7361.

89 M. L. Aubrey, R. Ameloot, B. M. Wiers and J. R. Long, Energy Environ. Sci., 2014, 7, 667.

90 P. Saha, M. K. Datta, O. I. Velikokhatnyi, A. Manivannan, D. Alman and P. N. Kumta, Prog. Mater. Sci., 2014, 66, 1-86.

91 X. J. Zhang, W. J. Wang, Z. J. Hu, G. N. Wang and K. S. Uvdal, Coord. Chem. Rev., 2015, 284, 206-235.

92 M. C. So, G. P. Wiederrecht, J. E. Mondloch, J. T. Hupp and O. K. Farha, Chem. Commun., 2015, 51, 3501-3510.

93 V. Stavila, A. A. Talin and M. D. Allendorf, Chem. Soc. Rev., 2014, 43, 5994-6010.

94 Y. J. Cui, Y. F. Yue, G. D. Qian and B. L. Chen, Chem. Rev., 2012, 112, 1126-1162.

95 C. J. Brabec, S. Gowrisanker, J. J. M. Halls, D. Laird, S. J. Jia and S. P. Williams, Adv. Mater., 2010, 22, 3839-3856.

96 B. E. Hardin, H. J. Snaith and M. D. McGehee, Nat. Photonics, 2012, 6, 162-169.

97 R. Kaur, K.-H. Kim, A. K. Paul and A. Deep, J. Mater. Chem. A, 2016, 4, 3991-4002.

98 M. E. Foster, J. D. Azoulay, B. M. Wong and M. D. Allendorf, Chem. Sci., 2014, 5, 2081-2090.

99 K. Leong, M. E. Foster, B. M. Wong, E. D. Spoerke, D. Van Gough, J. C. Deaton and M. D. Allendorf, J. Mater. Chem. A, 2014, 2, 3389.

100 Y. Zeng, Z. Y. Fu, H. J. Chen, C. C. Liu, S. J. Liao and J. C. Dai, Chem. Commun., 2012, 48, 8114-8116.

101 E. D. Spoerke, L. J. Small, M. E. Foster, J. Wheeler, A. M. Ullman, V. Stavila, M. Rodriguez and M. D. Allendorf, J. Phys. Chem. C, 2017, 121, 4816-4824.
102 A. V. Vinogradov, H. Zaake-Hertling, E. Hey-Hawkins, A. V. Agafonov, G. A. Seisenbaeva, V. G. Kessler and V. V. Vinogradov, Chem. Commun., 2014, 50, 10210.

103 W. A. Maza and A. J. Morris, J. Phys. Chem. C, 2014, 118, 8803-8817.

104 D. Y. Lee, E. K. Kim, C. Y. Shin, D. V. Shinde, W. Lee, N. K. Shrestha, J. K. Lee and S. H. Han, RSC Adv., 2014, 4, 12037-12042.

105 W. A. Maza, A. J. Haring, S. R. Ahrenholtz, C. C. Epley, S. Y. Lin and A. J. Morris, Chem. Sci., 2016, 7, 719-727.

106 W. A. Maza, S. R. Ahrenholtz, C. C. Epley, C. S. Day and A. J. Morris, J. Phys. Chem. C, 2014, 118, 14200-14210.

107 W. A. Maza, R. Padilla and A. J. Morris, J. Am. Chem. Soc., 2015, 137, 8161-8168.

108 G. Lu, O. K. Farha, W. Zhang, F. Huo and J. T. Hupp, Adv. Mater., 2012, 24, 3970-3974.

109 H. J. Son, S. Y. Jin, S. Patwardhan, S. J. Wezenberg, N. C. Jeong, M. So, C. E. Wilmer, A. A. Sarjeant, G. C. Schatz, R. Q. Snurr, O. K. Farha, G. P. Wiederrecht and J. T. Hupp, J. Am. Chem. Soc., 2013, 135, 862-869.

110 X. Du, R. Fan, X. Wang, G. Yu, L. Qiang, P. Wang, S. Gao and Y. Yang, Cryst. Growth Des., 2016, 16, 1737-1745.

111 D. Y. Lee, D. V. Shinde, S. J. Yoon, K. N. Cho, W. Lee, N. K. Shrestha and S.-H. Han, J. Phys. Chem. C, 2014, 118, 16328-16334.

112 F. X. Llabrés i Xamena, A. Corma and H. Garcia, J. Phys. Chem. C, 2007, 111, 80-85.

113 H. A. Lopez, A. Dhakshinamoorthy, B. Ferrer, P. Atienzar, M. Alvaro and H. Garcia, J. Phys. Chem. C, 2011, 115, 22200-22206.

114 Y. Li, A. Pang, C. Wang and M. Wei, J. Mater. Chem., 2011, 21, 17259-17264.

115 Y. Li, C. Chen, X. Sun, J. Dou and M. Wei, ChemSusChem, 2014, 7, 2469-2472.

116 T.-H. Chang, C.-W. Kung, H.-W. Chen, T.-Y. Huang, S.Y. Kao, H.-C. Lu, M.-H. Lee, K. M. Boopathi, C.-W. Chu and K.-C. Ho, Adv. Mater., 2015, 27, 7229-7235.

117 M. D. Allendorf, C. A. Bauer, R. K. Bhakta and R. J. T. Houk, Chem. Soc. Rev., 2009, 38, 1330-1352.

118 J. Heine and K. Muller-Buschbaum, Chem. Soc. Rev., 2013, 42, 9232-9242.

119 Z. Hu, B. J. Deibert and J. Li, Chem. Soc. Rev., 2014, 43, 5815-5840.

120 K. Hirai, P. Falcaro, S. Kitagawa and S. Furukawa, in MetalOrganic Frameworks for Photonics Applications, ed. B. Chen and G. Qian, 2014, vol. 157, pp. 167-186.

121 D. Jonckheere, E. Coutino-Gonzalez, W. Baekelant, B. Bueken, H. Reinsch, I. Stassen, O. Fenwick, F. Richard, P. Samori, R. Ameloot, J. Hofkens, M. B. J. Roeffaers and D. E. De Vos, J. Mater. Chem. C, 2016, 4, 4259-4268.

122 C. M. Doherty, D. Buso, A. J. Hill, S. Furukawa, S. Kitagawa and P. Falcaro, Acc. Chem. Res., 2014, 47, 396-405.

123 D. F. Sava, L. E. S. Rohwer, M. A. Rodriguez and T. M. Nenoff, J. Am. Chem. Soc., 2012, 134, 3983-3986.

124 W. P. Lustig, F. Wang, S. J. Teat, Z. Hu, Q. Gong and J. Li, Inorg. Chem., 2016, 55, 7250-7256. 
125 C.-Y. Sun, X.-L. Wang, X. Zhang, C. Qin, P. Li, Z.-M. Su, D.-X. Zhu, G.-G. Shan, K.-Z. Shao, H. Wu and J. Li, Nat. Commun., 2013, 4, 2717.

126 A. K. Chaudhari, M. R. Ryder and J.-C. Tan, Nanoscale, 2016, 8, 6851-6859.

127 Z. Hu, G. Huang, W. P. Lustig, F. Wang, H. Wang, S. J. Teat, D. Banerjee, D. Zhang and J. Li, Chem. Commun., 2015, 51, 3045-3048.

128 Q. Gong, Z. Hu, B. J. Deibert, T. J. Emge, S. J. Teat, D. Banerjee, B. Mussman, N. D. Rudd and J. Li, J. Am. Chem. Soc., 2014, 136, 16724-16727.

129 T. Panda and R. Banerjee, Proc. Natl. Acad. Sci. India Sect. Phys. Sci., 2014, 84, 331-336.

130 M. Kurmoo, Chem. Soc. Rev., 2009, 38, 1353-1379.

131 E. Coronado and G. Mínguez Espallargas, Chem. Soc. Rev., 2013, 42, 1525-1539.

132 E. Konig, G. Ritter and S. K. Kulshreshtha, Chem. Rev., 1985, 219-234.

133 P. Gütlich and H. A. Goodwin, Top. Curr. Chem., 2004, 1, 1-47.

134 P. Gütlich, A. Hauser and H. Spiering, Angew. Chem., Int. Ed. Engl., 1994, 33, 2024-2054.

135 G. J. Halder, C. J. Kepert, B. Moubaraki, K. S. Murray and J. D. Cashion, Science, 2002, 298, 1762-1765.

136 Y. Garcia, O. Kahn, L. Rabardel, B. Chansou, L. Salmon and J. P. Tuchagues, Inorg. Chem., 1999, 4663-4670.

137 S. M. Neville, B. Moubaraki, K. S. Murray and C. J. Kepert, Angew. Chem., Int. Ed., 2007, 46, 2059-2062.

138 S. M. Neville, G. J. Halder, K. W. Chapman, M. B. Duriska, P. D. Southon, J. D. Cashion, J. F. Letard, B. Moubaraki, K. S. Murray and C. J. Kepert, J. Am. Chem. Soc., 2008, 130, 2869-2876.

139 G. J. Halder, K. W. Chapman, S. M. Neville, B. Moubaraki, K. S. Murray, J. F. Letard and C. J. Kepert, J. Am. Chem. Soc., 2008, 130, 17552-17562.

140 S. M. Neville, G. J. Halder, K. W. Chapman, M. B. Duriska, B. Moubaraki, K. S. Murray and C. J. Kepert, J. Am. Chem. Soc., 2009, 131, 12106-12108.

141 J. Cirera, Rev. Inorg. Chem., 2014, 34, 199-216.

142 R. Ohtani and S. Hayami, Chem. - Eur. J., 2017, 23, 2236-2248.

143 G. Molnár, S. Cobo, J. A. Real, F. Carcenac, E. Daran, C. Vieu and A. Bousseksou, Adv. Mater., 2007, 19, 2163-2167.

144 S. Bonhommeau, G. Molnár, A. Galet, A. Zwick, J. A. Real, J. J. McGarvey and A. Bousseksou, Angew. Chem., Int. Ed., 2005, 44, 4069-4073.

145 T. Haraguchi, K. Otsubo, O. Sakata, A. Fujiwara and H. Kitagawa, Inorg. Chem., 2015, 54, 11593-11595.

146 D. Aravena, Z. A. Castillo, M. C. Munoz, A. B. Gaspar, K. Yoneda, R. Ohtani, A. Mishima, S. Kitagawa, M. Ohba, J. A. Real and E. Ruiz, Chem. - Eur. J., 2014, 20, 12864-12873.

147 C. H. Pham, J. Cirera and F. Paesani, J. Am. Chem. Soc., 2016, 138, 6123-6126.

148 C. Bartual-Murgui, L. Salmon, A. Akou, C. Thibault, G. Molnar, T. Mahfoud, Z. Sekkat, J. Antonio Real and A. Bousseksou, New J. Chem., 2011, 35, 2089-2094.
149 A. Akou, C. Bartual-Murgui, K. Abdul-Kader, M. Lopes, G. Molnar, C. Thibault, C. Vieu, L. Salmon and A. Bousseksou, Dalton Trans., 2013, 42, 16021-16028.

150 G. Agustí, S. Cobo, A. B. Gaspar, G. Molnár, N. O. Moussa, P. Á. Szilágyi, V. Pálfi, C. Vieu, M. Carmen Muñoz, J. A. Real and A. Bousseksou, Chem. Mater., 2008, 20, 6721-6732.

151 C. Bartual-Murgui, A. Akou, C. Thibault, G. Molnár, C. Vieu, L. Salmon and A. Bousseksou, J. Mater. Chem. C, 2015, 3, 1277-1285.

152 W. Volksen, R. D. Miller and G. Dubois, Chem. Rev., 2010, 110, 56-110.

153 K. Maex, M. R. Baklanov, D. Shamiryan, F. Lacopi, S. H. Brongersma and Z. S. Yanovitskaya, J. Appl. Phys., 2003, 93, 8793-8841.

154 S. Mendiratta, M. Usman, T.-T. Luo, S.-F. Lee, Y.-C. Lin and K.-L. Lu, CrystEngComm, 2014, 16, 6309.

155 K. Vanstreels, C. Wu and M. R. Baklanov, ECS J. Solid State Sci. Technol., 2014, 4, N3058-N3064.

156 A. Grill, S. M. Gates, T. E. Ryan, S. V. Nguyen and D. Priyadarshini, Appl. Phys. Rev., 2014, 1, 011306.

157 K. Zagorodniy, G. Seifert and H. Hermann, Appl. Phys. Lett., 2010, 97, 251905.

158 R. Warmbier, A. Quandt and G. Seifert, J. Phys. Chem. C, 2014, 118, 11799-11805.

159 M. Usman, S. Mendiratta and K.-L. Lu, ChemElectroChem, 2015, 2, 786-788.

160 S. Eslava, L. Zhang, S. Esconjauregui, J. Yang, K. Vanstreels, M. R. Baklanov and E. Saiz, Chem. Mater., 2013, 25, 27-33.

161 M. Usman, C.-H. Lee, D.-S. Hung, S.-F. Lee, C.-C. Wang, T.-T. Luo, L. Zhao, M.-K. Wu and K.-L. Lu, J. Mater. Chem. C, 2014, 2, 3762.

162 E. Redel, Z. Wang, S. Walheim, J. Liu, H. Gliemann and C. Wöll, Appl. Phys. Lett., 2013, 103, 091903.

163 D. Braga and G. Horowitz, Adv. Mater., 2009, 21, 1473-1486. 164 Y. Ha, J. D. Emery, M. J. Bedzyk, H. Usta, A. Facchetti and T. J. Marks, J. Am. Chem. Soc., 2011, 133, 10239-10250.

165 W.-J. Li, J. Liu, Z.-H. Sun, T.-F. Liu, J. Lü, S.-Y. Gao, C. He, R. Cao and J.-H. Luo, Nat. Commun., 2016, 7, 11830.

166 W. Zhang and R.-G. Xiong, Chem. Rev., 2012, 112, 1163-1195. 167 A. S. Tayi, A. Kaeser, M. Matsumoto, T. Aida and S. I. Stupp, Nat. Chem., 2015, 7, 281-294.

168 Handbook of semiconductor manufacturing technology, ed. R. Doering and Y. Nishi, CRC Press, Boca Raton, 2nd edn, 2008.

169 A. Hierlemann, O. Brand, C. Hagleitner and H. Baltes, Proc. IEEE, 2003, 91, 839-863.

170 Handbook of deposition technologies for films and coatings: science, applications and technology, ed. P. M. Martin, Elsevier, Amsterdam, 3rd edn, 2010.

171 S. M. George, Chem. Rev., 2010, 110, 111-131.

172 I. Stassen, D. De Vos and R. Ameloot, Chem. - Eur. J., 2016, 22, 14452-14460.

173 Handbook of thin film deposition: techniques, processes, and technologies, ed. K. Seshan, Elsevier, William Andrew, Amsterdam, Boston, Waltham, 3rd edn, 2012. 
174 A. Bétard and R. A. Fischer, Chem. Rev., 2012, 112, 1055-1083.

175 P. Falcaro, R. Ricco, C. M. Doherty, K. Liang, A. J. Hill and M. J. Styles, Chem. Soc. Rev., 2014, 43, 5513-5560.

176 J. Yao and H. Wang, Chem. Soc. Rev., 2014, 43, 4470-4493. 177 G. Lu and J. Hupp, J. Am. Chem. Soc., 2010, 132, 7832-7833.

178 D. Zacher, A. Baunemann, S. Hermes and R. Fischer, J. Mater. Chem., 2007, 17, 2785-2792.

179 A. Schoedel, C. Scherb and T. Bein, Angew. Chem., Int. Ed., 2010, 49, 7225-7228.

180 P. C. Lemaire, J. Zhao, P. S. Williams, H. J. Walls, S. D. Shepherd, M. D. Losego, G. W. Peterson and G. N. Parsons, ACS Appl. Mater. Interfaces, 2016, 8, 9514-9522.

181 N. Stock and S. Biswas, Chem. Rev., 2012, 2, 933-969.

182 J. Tao, X. Wang, T. Sun, H. Cai, Y. Wang, T. Lin, D. Fu, L. L. Y. Ting, Y. Gu and D. Zhao, Sci. Rep., 2017, 7, 41640.

183 S. Hermes, F. Schröder, R. Chelmowski, C. Wöll and R. A. Fischer, J. Am. Chem. Soc., 2005, 127, 13744-13745.

184 P. Falcaro, A. J. Hill, K. M. Nairn, J. Jasieniak, J. I. Mardel, T. J. Bastow, S. C. Mayo, M. Gimona, D. Gomez, H. J. Whitfield, R. Riccò, A. Patelli, B. Marmiroli, H. Amenitsch, T. Colson, L. Villanova and D. Buso, Nat. Commun., 2011, 2, 237.

185 E. Zanchetta, L. Malfatti, R. Ricco, M. J. Styles, F. Lisi, C. J. Coghlan, C. J. Doonan, A. J. Hill, G. Brusatin and P. Falcaro, Chem. Mater., 2015, 27, 690-699.

186 B. K. Keitz, C. J. Yu, J. R. Long and R. Ameloot, Angew. Chem., Int. Ed., 2014, 53, 5561-5565.

187 R. Ameloot, E. Gobechiya, H. Uji-i, J. A. Martens, J. Hofkens, L. Alaerts, B. F. Sels and D. E. De Vos, Adv. Mater., 2010, 22, 2685-2688.

188 C. Carbonell, I. Imaz and D. Maspoch, J. Am. Chem. Soc., 2011, 133, 2144-2147.

189 D. Witters, N. Vergauwe, R. Ameloot, S. Vermeir, D. De Vos, R. Puers, B. Sels and J. Lammertyn, Adv. Mater., 2012, 24, 1316-1320.

190 J.-L. Zhuang, D. Ar, X.-J. Yu, J.-X. Liu and A. Terfort, Adv. Mater., 2013, 25, 4631-4635.

191 D. Zacher, O. Shekhah, C. Wöll and R. A. Fischer, Chem. Soc. Rev., 2009, 38, 1418.

192 J. Liu, E. Redel, S. Walheim, Z. Wang, V. Oberst, J. Liu, S. Heissler, A. Welle, M. Moosmann, T. Scherer, M. Bruns, H. Gliemann and C. Wöll, Chem. Mater., 2015, 27, 1991-1996.

193 J. Liu, B. Lukose, O. Shekhah, H. K. Arslan, P. Weidler, H. Gliemann, S. Bräse, S. Grosjean, A. Godt, X. Feng, K. Müllen, I.-B. Magdau, T. Heine and C. Wöll, Sci. Rep., 2012, 2, 921.

194 Z. Wang, J. Liu, B. Lukose, Z. Gu, P. G. Weidler, H. Gliemann, T. Heine and C. Wöll, Nano Lett., 2014, 14, 1526-1529.

195 D. Zacher, K. Yusenko, A. Bétard, S. Henke, M. Molon, T. Ladnorg, O. Shekhah, B. Schüpbach, T. Dea Losa Arcos, M. Krasnopolski, M. Meilikhov, J. Winter, A. Terfort, C. Wöll and R. A. Fischer, Chem. - Eur. J., 2011, 17, 1448-1455.
196 B. Liu, M. Tu, D. Zacher and R. A. Fischer, Adv. Funct. Mater., 2013, 23, 3790-3798.

197 J.-L. Zhuang, A. Terfort and C. Wöll, Coord. Chem. Rev., 2016, 307, 391-424.

198 C. Scherb, A. Schödel and T. Bein, Angew. Chem., Int. Ed., 2008, 47, 5777-5779.

199 E. Biemmi, A. Darga, N. Stock and T. Bein, Microporous Mesoporous Mater., 2008, 114, 380-386.

200 O. Shekhah and M. Eddaoudi, Chem. Commun., 2013, 49, 10079.

201 A. Huang, W. Dou and J. Caro, J. Am. Chem. Soc., 2010, 132, 15562-15564.

202 H. K. Arslan, O. Shekhah, J. Wohlgemuth, M. Franzreb, R. A. Fischer and C. Woll, Adv. Funct. Mater., 2011, 21, 4228-4231.

203 V. Chernikova, O. Shekhah and M. Eddaoudi, ACS Appl. Mater. Interfaces, 2016, 8, 20459-20464.

204 Z. Wang, J. Liu, S. Grosjean, D. Wagner, W. Guo, Z. Gu, L. Heinke, H. Gliemann, S. Bräse and C. Wöll, ChemNanoMat, 2015, 1, 338-345.

205 Y. Hu, X. Dong, J. Nan, W. Jin, X. Ren, N. Xu and Y. M. Lee, Chem. Commun., 2011, 47, 737-739.

206 J. Reboul, S. Furukawa, N. Horike, M. Tsotsalas, K. Hirai, H. Uehara, M. Kondo, N. Louvain, O. Sakata and S. Kitagawa, Nat. Mater., 2012, 11, 717-723.

207 K. Khaletskaya, S. Turner, M. Tu, S. Wannapaiboon, A. Schneemann, R. Meyer, A. Ludwig, G. Van Tendeloo and R. A. Fischer, Adv. Funct. Mater., 2014, 24, 4804-4811.

208 N. Kornienko, Y. Zhao, C. S. Kley, C. Zhu, D. Kim, S. Lin, C. J. Chang, O. M. Yaghi and P. Yang, J. Am. Chem. Soc., 2015, 137, 14129-14135.

209 M. Drobek, J.-H. Kim, M. Bechelany, C. Vallicari, A. Julbe and S. S. Kim, ACS Appl. Mater. Interfaces, 2016, 8, 8323-8328.

210 I. Stassen, N. Campagnol, J. Fransaer, P. Vereecken, D. De Vos and R. Ameloot, CrystEngComm, 2013, 15, 9308.

211 I. Stassen, M. Styles, G. Grenci, H. V. Gorp, W. Vanderlinden, S. D. Feyter, P. Falcaro, D. D. Vos, P. Vereecken and R. Ameloot, Nat. Mater., 2016, 15, 304-310.

212 G. Majano and J. Pérez-Ramírez, Adv. Mater., 2013, 25, 1052-1057.

213 J. Zhao, W. T. Nunn, P. C. Lemaire, Y. Lin, M. D. Dickey, C. J. Oldham, H. J. Walls, G. W. Peterson, M. D. Losego and G. N. Parsons, J. Am. Chem. Soc., 2015, 137, 13756-13759.

214 P. Falcaro, K. Okada, T. Hara, K. Ikigaki, Y. Tokudome, A. W. Thornton, A. J. Hill, T. Williams, C. Doonan and M. Takahashi, Nat. Mater., 2016, 16, 342-348.

215 H. Ji, S. Hwang, K. Kim, C. Kim and N. C. Jeong, ACS Appl. Mater. Interfaces, 2016, 8, 32414-32420.

216 S. M. Yoon, J. H. Park and B. A. Grzybowski, Angew. Chem., Int. Ed., 2017, 56, 127-132.

217 Y. Chen, S. Li, X. Pei, J. Zhou, X. Feng, S. Zhang, Y. Cheng, H. Li, R. Han and B. Wang, Angew. Chem., Int. Ed., 2016, 55, 3419-3423.

218 H. Al-Kutubi, J. Gascon, E. J. R. Sudhölter and L. Rassaei, ChemElectroChem, 2015, 2, 462-474. 
219 W.-J. Li, M. Tu, R. Cao and R. A. Fischer, J. Mater. Chem. A, 2016, 4, 12356-12369.

220 U. Mueller, M. Schubert, F. Teich, H. Puetter, K. SchierleArndt and J. Pastré, J. Mater. Chem., 2006, 16, 626-636.

221 R. Ameloot, L. Stappers, J. Fransaer, L. Alaerts, B. F. Sels and D. E. Vos, Chem. Mater., 2009, 21, 2580-2582.

222 A. Martinez Joaristi, J. Juan-Alcañiz, P. Serra-Crespo, F. Kapteijn and J. Gascon, Cryst. Growth Des., 2012, 12, 3489-3498.

223 N. Campagnol, T. Van Assche, T. Boudewijns, J. Denayer, K. Binnemans, D. De Vos and J. Fransaer, J. Mater. Chem. A, 2013, 1, 5827.

224 P. Schäfer, M. A. van der Veen and K. F. Domke, Chem. Commun., 2016, 52, 4722-4725.

225 S. D. Worrall, H. Mann, A. Rogers, M. A. Bissett, M. P. Attfield and R. A. W. Dryfe, Electrochim. Acta, 2016, 197, 228-240.

226 I. Stassen, M. Styles, T. Van Assche, N. Campagnol, J. Fransaer, J. Denayer, J.-C. Tan, P. Falcaro, D. De Vos and R. Ameloot, Chem. Mater., 2015, 27, 1801-1807.

227 M. Li and M. Dincă, J. Am. Chem. Soc., 2011, 133, 12926-12929.

228 H. Liu, H. Wang, T. Chu, M. Yu and Y. Yang, J. Mater. Chem. C, 2014, 2, 8683-8690.

229 M. Li and M. Dincă, Chem. Sci., 2014, 5, 107.

230 M. Li and M. Dincă, Chem. Mater., 2015, 27, 3203-3206.

231 N. Campagnol, T. R. C. Van Assche, M. Li, L. Stappers, M. Dincă, J. F. M. Denayer, K. Binnemans, D. E. De Vos and J. Fransaer, J. Mater. Chem. A, 2016, 4, 3914-3925.

232 C. Hou, J. Peng, Q. Xu, Z. Ji and X. Hu, RSC Adv., 2012, 2, 12696.

233 N. Campagnol, I. Stassen, K. Binnemans, D. E. de Vos and J. Fransaer, J. Mater. Chem. A, 2015, 3, 19747-19753.

234 L.-L. Jiang, X. Zeng, M. Li, M.-Q. Wang, T.-Y. Su, X.-C. Tian and J. Tang, RSC Adv., 2017, 7, 9316-9320.

235 S. Yadnum, J. Roche, E. Lebraud, P. Négrier, P. Garrigue, D. Bradshaw, C. Warakulwit, J. Limtrakul and A. Kuhn, Angew. Chem., Int. Ed., 2014, 53, 4001-4005.

236 P. Horcajada, C. Serre, D. Grosso, C. Boissiere, S. Perruchas, C. Sanchez and G. Ferey, Adv. Mater., 2009, 21, 1931-1935.

237 A. Demessence, P. Horcajada, C. Serre, C. Boissière, D. Grosso, C. Sanchez and G. Férey, Chem. Commun., 2009, 7149-7151.

238 F. M. Hinterholzinger, A. Ranft, J. M. Feckl, B. Rühle, T. Bein and B. V. Lotsch, J. Mater. Chem., 2012, 22, 10356.

239 D.-Y. Kim, B. N. Joshi, J.-G. Lee, J.-H. Lee, J. S. Lee, Y. K. Hwang, J.-S. Chang, S. Al-Deyab, J.-C. Tan and S. S. Yoon, Chem. Eng. J., 2016, 295, 49-56.

240 T. Yamada, K. Otsubo, R. Makiura and H. Kitagawa, Chem. Soc. Rev., 2013, 42, 6655.

241 R. Ameloot, F. Vermoortele, W. Vanhove, M. B. J. Roeffaers, B. F. Sels and D. E. De Vos, Nat. Chem., 2011, 3, 382-387.

242 Y. Hwang, H. Sohn, A. Phan, O. M. Yaghi and R. N. Candler, Nano Lett., 2013, 13, 5271-5276.

243 I. Hod, W. Bury, D. M. Karlin, P. Deria, C.-W. Kung, M. J. Katz, M. So, B. Klahr, D. Jin, Y.-W. Chung,
T. W. Odom, O. K. Farha and J. T. Hupp, Adv. Mater., 2014, 26, 6295-6300.

244 C. M. Doherty, G. Grenci, R. Riccò, J. I. Mardel, J. Reboul, S. Furukawa, S. Kitagawa, A. J. Hill and P. Falcaro, $A d v$. Mater., 2013, 25, 4701-4705.

245 C. Dimitrakakis, B. Marmiroli, H. Amenitsch, L. Malfatti, P. Innocenzi, G. Grenci, L. Vaccari, A. J. Hill, B. P. Ladewig, M. R. Hill and P. Falcaro, Chem. Commun., 2012, 48, 7483-7485.

246 P. Innocenzi, L. Malfatti and P. Falcaro, Soft Matter, 2012, 8, 3722 .

247 M. Muller, O. I. Lebedev and R. A. Fischer, J. Mater. Chem., 2008, 5274-5281.

248 S. Proch, J. Herrmannsdorfer, R. Kempe, C. Kern, A. Jess, L. Seyfarth and J. Senker, Chem. - Eur. J., 2008, 8204-8212.

249 F. Schröder, D. Esken, M. Cokoja, M. W. E. van den Berg, O. I. Lebedev, G. Van Tendeloo, B. Walaszek, G. Buntkowsky, H.-H. Limbach, B. Chaudret and R. A. Fischer, J. Am. Chem. Soc., 2008, 130, 6119-6130.

250 F. Schroder, S. Henke, X. Zhang and R. A. Fischer, Eur. J. Inorg. Chem., 2009, 3131-3140.

251 M. S. El-Shall, V. Abdelsayed, A. E. R. S. Khder, H. M. A. Hassan, H. M. El-Kaderi and T. E. Reich, J. Mater. Chem., 2009, 19, 7625.

252 M. Sabo, A. Henschel, H. Fröde, E. Klemm and S. Kaskel, J. Mater. Chem., 2007, 17, 3827.

253 C. J. Doonan, W. Morris, H. Furukawa and O. M. Yaghi, J. Am. Chem. Soc., 2009, 131, 9492-9493.

254 T. Ishida, M. Nagaoka, T. Akita and M. Haruta, Chem. Eur. J., 2008, 14, 8456-8460.

255 T. Ishida, N. Kawakita, T. Akita and M. Haruta, Gold Bull., 2009, 42, 267-274.

256 H.-L. Jiang, B. Liu, T. Akita, M. Haruta, H. Sakurai and Q. Xu, J. Am. Chem. Soc., 2009, 131, 11302-11303.

257 B. W. Jacobs, R. J. T. Houk, M. R. Anstey, S. D. House, I. M. Robertson, A. A. Talin and M. D. Allendorf, Chem. Sci., 2011, 2, 411.

258 R. J. T. Houk, B. W. Jacobs, F. Gabaly, N. N. Chang, A. A. Talin, D. D. Graham, S. D. House, I. M. Robertson and M. D. Allendorf, Nano Lett., 2009, 9, 3413-3418.

259 B. Volosskiy, K. Niwa, Y. Chen, Z. Zhao, N. O. Weiss, X. Zhong and M. Ding, ACS Nano, 2015, 3044-3049.

260 M. Meilikhov, K. Yusenko, D. Esken, S. Turner, G. Van Tendeloo and R. A. Fischer, Eur. J. Inorg. Chem., 2010, 3701-3714.

261 F. Schroder and R. A. Fischer, Top. Curr. Chem., 2010, 293, 77-113.

262 C. R. Kim, T. Uemura and S. Kitagawa, Chem. Soc. Rev., 2016, 45, 1-18.

263 M. Müller, X. Zhang, Y. Wang and R. A. Fischer, Chem. Commun., 2009, 119-121.

264 D. Esken, H. Noei, Y. Wang, C. Wiktor, S. Turner, G. Tendeloo and R. A. Fischer, J. Mater. Chem., 2011, 21, 5907-5915.

265 D. Esken, S. Turner, C. Wiktor, S. B. Kalidindi, G. Tendeloo and R. A. Fischer, J. Am. Chem. Soc., 2011, 133, 16370-16373.

266 T. Wakaoka, K. Hirai, K. Murayama, Y. Takano, H. Takagi, S. Furukawa and S. Kitagawa, J. Mater. Chem. C, 2014, 2, 7173. 
267 S. Jin, H.-J. Son, O. K. Farha, G. P. Wiederrecht and J. T. Hupp, J. Am. Chem. Soc., 2013, 135, 955-958.

268 T. Uemura, N. Yanai and S. Kitagawa, Chem. Soc. Rev., 2009, 38, 1228-1236.

269 T. Wang, M. Farajollahi, S. Henke, T. Zhu, S. R. Bajpe, S. Sun, J. S. Barnard, J. S. Lee, J. D. W. Madden, A. K. Cheetham and S. K. Smoukov, Mater. Horiz., 2017, 4, 64-71.

270 M. W. A. MacLean, T. Kitao, T. Suga, M. Mizuno, S. Seki, T. Uemura and S. Kitagawa, Angew. Chem., Int. Ed., 2016, 55, 708-713.

271 C. Lu, T. Ben, S. Xu and S. Qiu, Angew. Chem., Int. Ed., 2014, 53, 6454-6458.

272 T. Kitao, S. Bracco, A. Comotti, P. Sozzani, M. Naito, S. Seki, T. Uemura and S. Kitagawa, J. Am. Chem. Soc., 2015, 137, 5231-5238.

273 T. Uemura, N. Uchida, A. Asano, A. Saeki, S. Seki, M. Tsujimoto, S. Isoda and S. Kitagawa, J. Am. Chem. Soc., 2012, 134, 8360-8363.

274 T. Uemura, R. Kitaura, Y. Ohta, M. Nagaoka and S. Kitagawa, Angew. Chem., Int. Ed., 2006, 45, 4112-4116.

275 T. Uemura, Y. Kadowaki, N. Yanai and S. Kitagawa, Chem. Mater., 2009, 21, 4096-4098.

276 J. Aguilera-Sigalat and D. Bradshaw, Coord. Chem. Rev., 2016, 307, 267-291.

277 G. Lu, S. Li, Z. Guo, O. K. Farha, B. G. Hauser, X. Qi, Y. Wang, X. Wang, S. Han, X. Liu, J. S. DuChene, H. Zhang, Q. Zhang, X. Chen, J. Ma, S. C. J. Loo, W. D. Wei, Y. Yang, J. T. Hupp and F. Huo, Nat. Chem., 2012, 4, 310-316.

278 B. P. Biswal, D. B. Shinde, V. K. Pillai and R. Banerjee, Nanoscale, 2013, 5, 10556-10561.

279 X. Lin, G. Gao, L. Zheng, Y. Chi and G. Chen, Anal. Chem., 2014, 86, 1223-1228.

280 R. Li, J. Hu, M. Deng, H. Wang, X. Wang, Y. Hu, H. L. Jiang, J. Jiang, Q. Zhang, Y. Xie and Y. Xiong, Adv. Mater., 2014, 26, 4783-4788.

281 R. Kaur, A. K. Paul and A. Deep, Forensic Sci. Int., 2014, 242, 88-93.

282 D. Buso, J. Jasieniak, M. D. H. Lay, P. Schiavuta, P. Scopece, J. Laird, H. Amenitsch, A. J. Hill and P. Falcaro, Small, 2012, 8, 80-88.

283 J. Juan-Alcaniz, J. Gascon and F. Kapteijn, J. Mater. Chem., 2012, 22, 10102-10118.

284 Z. Zhang and M. J. Zaworotko, Chem. Soc. Rev., 2014, 5444-5455.

285 H. Yang, P. E. Kruger and S. G. Telfer, Inorg. Chem., 2015, 54, 9483-9490.

286 B. Liu, H. Shioyama, T. Akita and Q. Xu, J. Am. Chem. Soc., 2008, 130, 5390-5391.

287 J. Hu, H. Wang, Q. Gao and H. Guo, Carbon, 2010, 48, 3599-3606.

288 W. Chaikittisilp, K. Ariga and Y. Yamauchi, J. Mater. Chem. A, 2013, 1, 14-19.

289 A. J. Amali, J.-K. Sun and Q. Xu, Chem. Commun., 2014, 50, 1519-1522.

290 E. Proietti, F. Jaouen, M. Lefèvre, N. Larouche, J. Tian, J. Herranz and J.-P. Dodelet, Nat. Commun., 2011, 2, 416.
291 J. Tian, A. Morozan, M. T. Sougrati, M. Lefèvre, R. Chenitz, J.-P. Dodelet, D. Jones and F. Jaouen, Angew. Chem., Int. Ed., 2013, 52, 6867-6870.

292 L. Hu and Q. Chen, Nanoscale, 2014, 6, 1236-1257.

293 Y. Song, X. Li, L. Sun and L. Wang, RSC Adv., 2015, 5, 7267-7279.

294 J. C. Tan and A. K. Cheetham, Chem. Soc. Rev., 2011, 40, 1059-1080.

295 W. Li, S. Henke and A. K. Cheetham, APL Mater., 2014, 2, 123902.

296 J. C. Tan, J. D. Furman and A. K. Cheetham, J. Am. Chem. Soc., 2009, 131, 14252-14254.

297 W. Li, M. R. Probert, M. Kosa, T. D. Bennett, A. Thirumurugan, R. P. Burwood, M. Parinello, J. A. K. Howard and A. K. Cheetham, J. Am. Chem. Soc., 2012, 134, 11940-11943.

298 K. J. Gagnon, C. M. Beavers and A. Clearfield, J. Am. Chem. Soc., 2013, 135, 1252-1255.

299 K. W. Chapman, G. J. Halder and P. J. Chupas, J. Am. Chem. Soc., 2009, 131, 17546-17547.

300 A. U. Ortiz, A. Boutin, A. H. Fuchs and F.-X. Coudert, Phys. Rev. Lett., 2012, 109, 195502.

301 A. Schneemann, V. Bon, I. Schwedler, I. Senkovska, S. Kaskel and R. A. Fischer, Chem. Soc. Rev., 2014, 43, 6062-6096.

302 F.-X. Coudert, Chem. Mater., 2015, 27, 1905-1916.

303 C. Serre, F. Millange, C. Thouvenot, M. Nogues, G. Marsolier, F. Millange, C. Thouvenot, M. Nogus, G. Marsolier, D. Lour and G. Frey, J. Am. Chem. Soc., 2002, 124, 13519-13526.

304 C. Gücüyener, J. van den Bergh, J. Gascon and F. Kapteijn, J. Am. Chem. Soc., 2010, 132, 17704-17706.

305 F. Salles, G. Maurin, C. Serre, P. L. Llewellyn, C. Knöfel, H. J. Choi, Y. Filinchuk, L. Oliviero, A. Vimont, J. R. Long and others, J. Am. Chem. Soc., 2010, 132, 13782-13788.

306 S. Krause, V. Bon, I. Senkovska, U. Stoeck, D. Wallacher, D. M. Többens, S. Zander, R. S. Pillai, G. Maurin, F.-X. Coudert and S. Kaskel, Nature, 2016, 532, 348-352.

307 T. D. Bennett, A. K. Cheetham, A. H. Fuchs and F.-X. Coudert, Nat. Chem., 2016, 9, 11-16.

308 B. Van de Voorde, I. Stassen, B. Bueken, F. Vermoortele, D. De Vos, R. Ameloot, J.-C. Tan and T. D. Bennett, J. Mater. Chem. A, 2015, 3, 1737-1742.

309 S. Bundschuh, O. Kraft, H. K. Arslan, H. Gliemann, P. G. Weidler and C. Wöll, Appl. Phys. Lett., 2012, 101, 101910.

310 B. Van de Voorde, R. Ameloot, I. Stassen, M. Everaert, D. De Vos and J.-C. Tan, J. Mater. Chem. C, 2013, 1, 7716.

311 A. K. Cheetham, J. C. Tan and T. D. Bennett, Proc. Natl. Acad. Sci. U. S. A., 2010, 107, 9938-9943.

312 M. Kondo, S. Furukawa, K. Hirai, T. Tsuruoka, J. Reboul, H. Uehara, S. Diring, Y. Sakata, O. Sakata and S. Kitagawa, J. Am. Chem. Soc., 2014, 136, 4938-4944.

313 J. Lewis, Mater. Today, 2006, 9, 38-45.

314 Y. Liu, H. Wang, W. Shi, W. Zhang, J. Yu, B. K. Chandran, C. Cui, B. Zhu, Z. Liu, B. Li, C. Xu, Z. Xu, S. Li, W. Huang, F. Huo and X. Chen, Angew. Chem., Int. Ed., 2016, 55, 8884-8888. 
315 L. Pan, Z. Ji, X. Yi, X. Zhu, X. Chen, J. Shang, G. Liu and R.-W. Li, Adv. Funct. Mater., 2015, 25, 2677-2685.

316 J. A. Thornton and D. W. Hoffman, Thin Solid Films, 1989, 171, 5-31.

317 P. Lightfoot, D. A. Woodcock, M. J. Maple, L. A. Villaescusa and P. A. Wright, J. Mater. Chem., 2001, 11, 212-216.

318 Y. Wu, A. Kobayashi, G. Halder, V. Peterson, K. Chapman, N. Lock, P. Southon and C. Kepert, Angew. Chem., 2008, 120, 9061-9064.

319 W. Zhou, H. Wu, T. Yildirim, J. R. Simpson and A. R. H. Walker, Phys. Rev. B: Condens. Matter Mater. Phys., 2008, 78, 054114.

320 L. D. Devries, P. M. Barron, E. P. Hurley, C. Hu and W. Choe, J. Am. Chem. Soc., 2011, 133, 1484-14851.

321 L. Zhang, X. Kuang, X. Wu, W. Yang and C. Lu, Dalton Trans., 2014, 43, 7146-7152.

322 P. Lama, R. K. Das, V. J. Smith and L. J. Barbour, Chem. Commun., 2014, 50, 6464-6467.

323 L. Bouëssel du Bourg, A. U. Ortiz, A. Boutin and F.-X. Coudert, APL Mater., 2014, 2, 124110.

324 M. J. Cliffe, J. A. Hill, C. A. Murray, F.-X. Coudert and A. L. Goodwin, Phys. Chem. Chem. Phys., 2015, 17, 11586-11592.

325 C. A. Swenson, J. Phys. Chem. Ref. Data, 1983, 12, 179.

326 R. Buffington and W. Latimer, Coeff. Expans., 1926, 48, 2305-2319.

327 K. Takenaka, Sci. Technol. Adv. Mater., 2012, 13, 013001.

328 N. C. Burtch, D. Dubbeldam and K. S. Walton, Mol. Simul., 2015, 41, 1379-1387.

329 J. A. Greathouse and M. D. Allendorf, J. Phys. Chem. C, 2008, 112, 5795-5802.

330 D. Dubbeldam, K. S. Walton, D. E. Ellis and R. Q. Snurr, Angew. Chem., 2007, 46, 4496-4499.

331 S. S. Han and W. A. Goddard, J. Phys. Chem. C, 2007, 111, 15185-15191.

332 F. Shen, P. Lu, S. J. O'Shea, K. H. Lee and T. Y. Ng, Sens. Actuators, A, 2001, 95, 17-23.

333 Y. Liu, J. H. Her, A. Dailly, A. J. Ramirez-Cuesta, D. A. Neumann and C. M. Brown, J. Am. Chem. Soc., 2008, 130, 11813-11818.

334 A. M. Walker, B. Civalleri, B. Slater, C. Mellot-Draznieks, F. Corà, C. M. Zicovich-Wilson, G. Román-Pérez, J. M. Soler and J. D. Gale, Angew. Chem., Int. Ed., 2010, 49, 7501-7503.

335 L. E. Kreno, K. Leong, O. K. Farha, M. Allendorf, R. P. Van Duyne and J. T. Hupp, Chem. Rev., 2012, 112, 1105-1125.

336 J. Mahmood, E. K. Lee, M. Jung, D. Shin, I.-Y. Jeon, S.-M. Jung, H.-J. Choi, J.-M. Seo, S.-Y. Bae, S.-D. Sohn, N. Park, J. H. Oh, H.-J. Shin and J.-B. Baek, Nat. Commun., 2015, 6, 6486.

337 J. I. Feldblyum, C. H. McCreery, S. C. Andrews, T. Kurosawa, E. J. G. Santos, V. Duong, L. Fang, A. L. Ayzner and Z. Bao, Chem. Commun., 2015, 51, 13894-13897.

338 D. B. Strukov, G. S. Snider, D. R. Stewart and R. S. Williams, Nature, 2008, 453, 80-83.

339 A. A. Bessonov, M. N. Kirikova, D. I. Petukhov, M. Allen, T. Ryhänen and M. J. A. Bailey, Nat. Mater., 2014, 14, 199-204.
340 X. Huang, B. Zheng, Z. Liu, C. Tan, J. Liu, B. Chen, H. Li, J. Chen, X. Zhang, Z. Fan, W. Zhang, Z. Guo, F. Huo, Y. Yang, L.-H. Xie, W. Huang and H. Zhang, ACS Nano, 2014, 8, 8695-8701.

341 S. M. Yoon, S. C. Warren and B. A. Grzybowski, Angew. Chem., Int. Ed. Engl., 2014, 53, 4437-4441.

342 R. Waser and M. Aono, Nat. Mater., 2007, 6, 833-840.

343 Z. Wang, D. Nminibapiel, P. Shrestha, J. Liu, W. Guo, P. G. Weidler, H. Baumgart, C. Woell and E. Redel, ChemNanoMat, 2016, 2, 67-73.

344 S. D. Worrall, M. A. Bissett, W. Hirunpinyopas, M. Attfield and R. Dryfe, J. Mater. Chem. C, 2016, 4, 8687-8695.

345 World Health Organization, WHO guidelines for indoor air quality: selected pollutants, WHO, Copenhagen, 2010.

346 C. Di Natale, R. Paolesse, E. Martinelli and R. Capuano, Anal. Chim. Acta, 2014, 824, 1-17.

347 P. Kumar, L. Morawska, C. Martani, G. Biskos, M. Neophytou, S. Di Sabatino, M. Bell, L. Norford and R. Britter, Environ. Int., 2015, 75, 199-205.

348 H. Adam, G. Stanislaw and I. Folke, Pure Appl. Chem., 1991, 63, 1247-1250.

349 G. Korotcenkov, Handbook of Gas Sensor Materials: Properties, Advantages and Shortcomings for Applications Volume 1: Conventional Approaches, Springer New York, New York, NY, 2013.

350 X. Li and D.-W. Lee, Meas. Sci. Technol., 2012, 23, 022001.

351 S. Vashist, J. Nanotechnol., 2007, 3, 1-15.

352 H. K. Hunt and A. M. Armani, Nanoscale, 2010, 2, 1544-1559.

353 B. N. Johnson and R. Mutharasan, Biosens. Bioelectron., 2012, 32, 1-18.

354 A. Alodhayb, S. M. S. Rahman, S. Rahman, P. E. Georghiou and L. Y. Beaulieu, Sens. Actuators, B, 2016, 237, 459-469.

355 M. D. Allendorf, R. J. T. Houk, L. Andruszkiewicz, A. A. Talin, J. Pikarsky, A. Choudhury, K. A. Gall and P. J. Hesketh, J. Am. Chem. Soc., 2008, 130, 14404-14405.

356 I. Ellern, A. Venkatasubramanian, J. H. Lee, P. Hesketh, V. Stavila, A. Robinson and M. Allendorf, Micro Nano Lett., 2013, 8, 766-769.

357 I. Ellern, A. Venkatasubramanian, J. H. Lee, P. J. Hesketh, V. Stavila, M. D. Allendorf and A. L. Robinson, ECS Transactions, 2012, vol. 50, pp. 469-476.

358 J.-H. Lee, R. T. J. Houk, A. Robinson, J. A. Greathouse, S. M. Thornberg, M. D. Allendorf and P. J. Hesketh, Proc. of SPIE, (Micro- and Nanotechnology Sensors, Systems, and Applications II), 2010, vol. 7679, p. 767927.

359 H. Yamagiwa, S. Sato, T. Fukawa, T. Ikehara, R. Maeda, T. Mihara and M. Kimura, Sci. Rep., 2014, 4, 6247.

360 C. Yim, M. Lee, W. Kim, S. Lee, G.-H. Kim, K. T. Kim and S. Jeon, Chem. Commun., 2015, 51, 6168-6171.

361 C. Yim, M. Lee, M. Yun, G.-H. Kim, K. T. Kim and S. Jeon, Sci. Rep., 2015, 5, 10674.

362 A. Venkatasubramanian, J. Lee, V. Stavila, A. Robinson, M. D. Allendorf and P. J. Hesketh, Sens. Actuators, B, 2012, 168, 256-262.

363 C. Prestipino, L. Regli, J. G. Vitillo, F. Bonino, A. Damin, C. Lamberti, A. Zecchina, P. L. Solari, K. O. Kongshaug and S. Bordiga, Chem. Mater., 2006, 18, 1337-1346. 
364 S. Devautour-Vinot, G. Maurin, F. Henn, C. Serre and G. Férey, Phys. Chem. Chem. Phys., 2010, 12, 12478-12485.

365 F. Coudert, A. Boutin, A. H. Fuchs and A. Neimark, J. Phys. Chem. Lett., 2013, 3198-3205.

366 S. Sakaida, K. Otsubo, O. Sakata, C. Song, A. Fujiwara, M. Takata and H. Kitagawa, Nat. Chem., 2016, 8, 377-383.

367 C. Zhang, J. A. Gee, D. S. Sholl and R. P. Lively, J. Phys. Chem. C, 2014, 118, 20727-20733.

368 Y. Hwang, A. Phan, K. Galatsis, O. M. Yaghi and R. N. Candler, J. Micromech. Microeng., 2013, 23, 125027.

369 A. Bouchaala, N. Jaber, O. Shekhah, V. Chernikova, M. Eddaoudi and M. I. Younis, Appl. Phys. Lett., 2016, 109, 013502.

370 A. Bouchaala, N. Jaber, O. Yassine, O. Shekhah, V. Chernikova, M. Eddaoudi and M. Younis, Sensors, 2016, 16, 758.

371 G. Sauerbrey, Z. Phys., 1959, 2, 206-222.

372 M. Madou, From MEMS to Bio-MEMS and Bio-NEMS: Manufacturing Techniques and Applications, CRC Press, Boca Raton, FL, 3rd edn, 2011.

373 L. Heinke, Z. Gu and C. Wöll, Nat. Commun., 2014, 5, 4562. 374 X. Si, C. Jiao, F. Li, J. Zhang, S. Wang, S. Liu, Z. Li, L. Sun, F. Xu, Z. Gabelica and C. Schick, Energy Environ. Sci., 2011, 4, 4522-4527.

375 M. Tu, S. Wannapaiboon, K. Khaletskaya and R. A. Fischer, Adv. Funct. Mater., 2015, 25, 4470-4479.

376 M. Meilikhov, S. Furukawa, K. Hirai, R. A. Fischer and S. Kitagawa, Angew. Chem., Int. Ed. Engl., 2013, 52, 341-345.

377 M. Tu, S. Wannapaiboon and R. A. Fischer, Dalton Trans., 2013, 42, 16029.

378 S. Wannapaiboon, M. Tu and R. A. Fischer, Adv. Funct. Mater., 2014, 24, 2696-2705.

379 M. Tu and R. A. Fischer, J. Mater. Chem. A, 2014, 2, 2018-2022.

380 S. Wannapaiboon, M. Tu, K. Sumida, K. Khaletskaya, S. Furukawa, S. Kitagawa and R. A. Fischer, J. Mater. Chem. A, 2015, 3, 23385-23394.

381 O. Zybaylo, O. Shekhah, H. Wang, M. Tafipolsky, R. Schmid, D. Johannsmann and C. Woll, Phys. Chem. Chem. Phys., 2010, 12, 8093-8098.

382 H. Uehara, S. Diring, S. Furukawa, Z. Kalay, M. Tsotsalas, M. Nakahama, K. Hirai, M. Kondo, O. Sakata and S. Kitagawa, J. Am. Chem. Soc., 2011, 133, 11932-11935.

383 B. Liu, M. Tu and R. A. Fischer, Angew. Chem., Int. Ed., 2013, 52, 3402-3405.

384 L. Heinke and C. Wöll, Phys. Chem. Chem. Phys., 2013, 15, 9295-9299.

385 L. Heinke, M. Cakici, M. Dommaschk, S. Grosjean, R. Herges, S. Bräse and C. Wöll, ACS Nano, 2014, 8, 1463-1467.

386 O. Shekhah, H. Wang, S. Kowarik, F. Schreiber, M. Paulus, M. Tolan, C. Sternemann, F. Evers, D. Zacher, R. A. Fischer and others, J. Am. Chem. Soc., 2007, 129, 15118-15119.

387 V. Stavila, J. Volponi, A. M. Katzenmeyer, M. C. Dixon and M. D. Allendorf, Chem. Sci., 2012, 3, 1531.

388 V. Stavila, C. Schneider, C. Mowry, T. R. Zeitler, J. A. Greathouse, A. L. Robinson, J. M. Denning, J. Volponi, K. Leong, W. Quan, M. Tu, R. A. Fischer and M. D. Allendorf, Adv. Funct. Mater., 2016, 26, 1699-1707.
389 H. Wohltjen and R. E. Dessy, Anal. Chem., 1979, 51, 1458. 390 L. Rayleigh, Proc. London Math. Soc., 1885, 17, 4-11.

391 A. L. Robinson, V. Stavila, T. R. Zeitler, M. I. White, S. M. Thornberg, J. A. Greathouse and M. D. Allendorf, Anal. Chem., 2012, 84, 7043-7051.

392 D. S. Ballantine, R. M. White, S. J. Martin, A. J. Ricco, E. T. Zellers, G. C. Frye and H. Wohltjen, Acoustic Wave Sensors: Theory, Design and Physico-Chemical Applications, Academic Press, San Diego, CA, 1997.

393 J. Janata, Principles of chemical sensors, Springer, New York, NY, 2nd edn, 2009.

394 H. Bai and G. Shi, Sensors, 2007, 7, 267-307.

395 Y. Liu, X. Dong and P. Chen, Chem. Soc. Rev., 2012, 41, 2283-2307.

396 A. Goldoni, L. Petaccia, S. Lizzit and R. Larciprete, J. Phys.: Condens. Matter, 2010, 22, 013001.

397 M.-S. Yao, W.-X. Tang, G.-E. Wang, B. Nath and G. Xu, Adv. Mater., 2016, 28, 5229-5234.

398 H. Tian, H. Fan, M. Li and L. Ma, ACS Sens., 2016, 1, 243-250. 399 N. A. Travlou, K. Singh, E. Rodríguez-Castellón and T. J. Bandosz, J. Mater. Chem. A, 2015, 3, 11417-11429.

400 B. Le Ouay, M. Boudot, T. Kitao, T. Yanagida, S. Kitagawa and T. Uemura, J. Am. Chem. Soc., 2016, 138, 10088-10091.

401 E.-X. Chen, H. Yang and J. Zhang, Inorg. Chem., 2014, 53, 5411-5413.

402 E.-X. Chen, H.-R. Fu, R. Lin, Y.-X. Tan and J. Zhang, ACS Appl. Mater. Interfaces, 2014, 6, 22871-22875.

403 M. G. Campbell, S. F. Liu, T. M. Swager and M. Dincă, J. Am. Chem. Soc., 2015, 137, 13780-13783.

404 S. Achmann, G. Hagen, J. Kita, I. M. Malkowsky, C. Kiener and R. Moos, Sensors, 2009, 9, 1574-1589.

405 Y. Zhang, Y. Chen, Y. Zhang, H. Cong, B. Fu, S. Wen and S. Ruan, J. Nanopart. Res., 2013, 15, 2014.

406 J. Liu, F. Sun, F. Zhang, Z. Wang, R. Zhang, C. Wang and S. Qiu, J. Mater. Chem., 2011, 21, 3775.

407 S. Homayoonnia and S. Zeinali, Sens. Actuators, B, 2016, 237, 776-786.

408 A. Weiss, N. Reimer, N. Stock, M. Tiemann and T. Wagner, Phys. Chem. Chem. Phys., 2015, 17, 21634-21642.

409 A. Weiss, N. Reimer, N. Stock, M. Tiemann and T. Wagner, Microporous Mesoporous Mater., 2016, 220, 39-43.

410 D. Fröhlich, E. Pantatosaki, P. D. Kolokathis, K. Markey, H. Reinsch, M. Baumgartner, M. A. van der Veen, D. E. De Vos, N. Stock, G. K. Papadopoulos, S. K. Henninger and C. Janiak, J. Mater. Chem. A, 2016, 4, 11859-11869.

411 S. Sachdeva, D. Soccol, D. J. Gravesteijn, F. Kapteijn, E. J. R. Sudhölter, J. Gascon and L. C. P. M. de Smet, ACS Sens., 2016, 1, 1188-1192.

412 O. Yassine, O. Shekhah, A. H. Assen, Y. Belmabkhout, K. N. Salama and M. Eddaoudi, Angew. Chem., Int. Ed., 2016, 55, 15879-15883.

413 A. Kahn, Mater. Horiz., 2016, 3, 7-10.

414 J. Janata and M. Josowicz, Anal. Chem., 1997, 69, 293A-296A.

415 J. Janata and M. Josowicz, Acc. Chem. Res., 1998, 31, 241-248.

416 A. Oprea, N. Bârsan and U. Weimar, Sens. Actuators, B, 2009, 142, 470-493. 
417 M. Hoerter, A. Oprea, N. Barsan and U. Weimar, Sens. Actuators, B, 2008, 134, 266-272.

418 P. Davydovskaya, R. Pohle, A. Tawil and M. Fleischer, Sens. Actuators, B, 2013, 187, 142-146.

419 P. Davydovskaya, A. Ranft, B. V. Lotsch and R. Pohle, Anal. Chem., 2014, 86, 6948-6958.

420 P. Davydovskaya, A. Ranft, B. V. Lotsch and R. Pohle, Procedia Eng., 2014, 87, 1433-1436.

421 V. Pentyala, P. Davydovskaya, R. Pohle, G. Urban and O. Yurchenko, Procedia Eng., 2014, 87, 1071-1074.

422 R. Pohle, A. Tawil, P. Davydovskaya and M. Fleischer, Procedia Eng., 2011, 25, 108-111.

423 I. Stassen, B. Bueken, H. Reinsch, J. F. M. Oudenhoven, D. Wouters, J. Hajek, V. Van Speybroeck, N. Stock, P. M. Vereecken, R. Van Schaijk, D. De Vos and R. Ameloot, Chem. Sci., 2016, 7, 5827-5832.

424 M. J. Katz, S.-Y. Moon, J. E. Mondloch, M. H. Beyzavi, C. J. Stephenson, J. T. Hupp and O. K. Farha, Chem. Sci., 2015, 6, 2086-2091.

425 A. M. Plonka, Q. Wang, W. O. Gordon, A. Balboa, D. Troya, W. Guo, C. H. Sharp, S. D. Senanayake, J. R. Morris, C. L. Hill and A. I. Frenkel, J. Am. Chem. Soc., 2017, 139, 599-602.

426 T.-H. Zhang, D. Petelenz and J. Janata, Sens. Actuators, B, 1993, 12, 175-180.

427 B. Flietner, T. Doll, J. Lechner, M. Leu and I. Eisele, Sens. Actuators, B, 1994, 22, 109-113.

428 B. J. Polk, in Sensors, 2002. Proceedings of IEEE, IEEE, 2002, vol. 1, pp. 732-735.

429 J. Janata, Electroanalysis, 2004, 16, 1831-1835.

430 A. Cao, W. Zhu, J. Shang, J. H. Klootwijk, E. J. R. Sudhölter, J. Huskens and L. C. P. M. de Smet, Nano Lett., 2017, 17, 1-7.

431 S. G. Surya, S. S. Nagarkar, S. K. Ghosh, P. Sonar and V. Ramgopal Rao, Sens. Actuators, B, 2016, 223, 114-122.

432 J. R. Stetter and J. Li, Chem. Rev., 2008, 108, 352-366.

433 J. Zhou, X. Li, L. Yang, S. Yan, M. Wang, D. Cheng, Q. Chen, Y. Dong, P. Liu, W. Cai and C. Zhang, Anal. Chim. Acta, 2015, 899, 57-65.

434 H. Hosseini, H. Ahmar, A. Dehghani, A. Bagheri, A. R. Fakhari and M. M. Amini, Electrochim. Acta, 2013, 88, 301-309.

435 E. Shi, H. Lin, Q. Wang, F. Zhang, S. Shi, T. Zhang, X. Li, H. Niu and F. Qu, Dalton Trans., 2017, 46, 554-563.

436 X. Wang, Q. Wang, Q. Wang, F. Gao, F. Gao, Y. Yang and H. Guo, ACS Appl. Mater. Interfaces, 2014, 6, 11573-11580.

437 M.-Q. Wang, C. Ye, S.-J. Bao, Y. Zhang, Y.-N. Yu and M. Xu, Analyst, 2016, 141, 1279-1285.

438 W. Zhang, J. Chen, Y. Li, W. Yang, Y. Zhang and Y. Zhang, RSC Adv., 2017, 7, 5628-5635.

439 H. Hosseini, H. Ahmar, A. Dehghani, A. Bagheri, A. Tadjarodi and A. R. Fakhari, Biosens. Bioelectron., 2013, 42, 426-429.

440 Y. Li, C. Huangfu, H. Du, W. Liu, Y. Li and J. Ye, J. Electroanal. Chem., 2013, 709, 65-69.

441 Y. Wang, Y. Zhang, C. Hou and M. Liu, RSC Adv., 2015, 5, 98260-98268.

442 Y. Wang, H. Ge, G. Ye, H. Chen and X. Hu, J. Mater. Chem. $B, 2015,3,3747-3753$.
443 Y. Wang, H. Chen, X. Hu and H. Yu, Analyst, 2016, 141, 4647-4653.

444 W. Ma, Q. Jiang, P. Yu, L. Yang and L. Mao, Anal. Chem., 2013, 85, 7550-7557.

445 W. Zhan, Q. Kuang, J. Zhou, X. Kong, Z. Xie and L. Zheng, J. Am. Chem. Soc., 2013, 1926-1933.

446 Z. Xu, L. Yang and C. Xu, Anal. Chem., 2015, 87, 3438-3444.

447 Z. Peng, Z. Jiang, X. Huang and Y. Li, RSC Adv., 2016, 6, 13742-13748.

448 T. Q. N. Tran, G. Das and H. H. Yoon, Sens. Actuators, B, 2017, 243, 78-83.

449 J. J. Gassensmith, J. Y. Kim, J. M. Holcroft, O. K. Farha, J. F. Stoddart, J. T. Hupp and N. C. Jeong, J. Am. Chem. Soc., 2014, 136, 8277-8282.

450 M. Hochberg and T. Baehr-Jones, Nat. Photonics, 2010, 4, 492-494.

451 F.-Y. Yi, D. Chen, M.-K. Wu, L. Han and H.-L. Jiang, ChemPlusChem, 2016, 81, 675-690.

452 S.-I. Ohira, Y. Miki, T. Matsuzaki, N. Nakamura, Y. Sato, Y. Hirose and K. Toda, Anal. Chim. Acta, 2015, 886, 188-193.

453 A. Ranft, F. Niekiel, I. Pavlichenko, N. Stock and B. V. Lotsch, Chem. Mater., 2015, 27, 1961-1970.

454 Z. Hu, C. Tao, F. Wang, X. Zou and J. Wang, J. Mater. Chem. $C, 2015,3,211-216$.

455 O. Dalstein, D. R. Ceratti, C. Boissière, D. Grosso, A. Cattoni and M. Faustini, Adv. Funct. Mater., 2016, 26, 81-90.

456 Y. Wu, F. Li, W. Zhu, J. Cui, C. Tao, C. Lin, P. M. Hannam and G. Li, Angew. Chem., Int. Ed., 2011, 50, 12518-12522.

457 G. Lu, O. K. Farha, L. E. Kreno, P. M. Schoenecker, K. S. Walton, R. P. Van Duyne and J. T. Hupp, Adv. Mater., 2011, 23, 4449-4452.

458 C. Cui, Y. Liu, H. Xu, S. Li, W. Zhang, P. Cui and F. Huo, Small Weinh. Bergstr. Ger., 2014, 10, 3672-3676.

459 L. Li, X. Jiao, D. Chen, B. V. Lotsch and C. Li, Chem. Mater., 2015, 27, 7601-7609.

460 C. Li, L. Li, S. Yu, X. Jiao and D. Chen, Adv. Mater. Technol., 2016, 1, 1600127.

461 O. Tokel, F. Inci and U. Demirci, Chem. Rev., 2014, 114, 5728-5752.

462 L. E. Kreno, J. T. Hupp and R. P. Van Duyne, Anal. Chem., 2010, 82, 8042-8046.

463 K.-J. Kim, X. Chong, P. B. Kreider, G. Ma, P. R. Ohodnicki, J. P. Baltrus, A. X. Wang and C.-H. Chang, J. Mater. Chem. C, 2015, 3, 2763-2767.

464 K. Sumida, D. L. Rogow, J. A. Mason, T. M. McDonald, E. D. Bloch, Z. R. Herm, T.-H. Bae and J. R. Long, Chem. Rev., 2012, 112, 724-781.

465 L. E. Kreno, N. G. Greeneltch, O. K. Farha, J. T. Hupp and R. P. Van Duyne, Analyst, 2014, 139, 4073.

466 Y. Zhao, N. Kornienko, Z. Liu, C. Zhu, S. Asahina, T.R. Kuo, W. Bao, C. Xie, A. Hexemer, O. Terasaki, P. Yang and O. M. Yaghi, J. Am. Chem. Soc., 2015, 137, 2199-2202.

467 J. Liao, D. Wang, A. Liu, Y. Hu and G. Li, Analyst, 2015, 140, 8165-8171. 
468 Y. Hu, J. Liao, D. Wang and G. Li, Anal. Chem., 2014, 86, 3955-3963.

469 M. Usman, S. Mendiratta and K.-L. Lu, Adv. Mater., 2017, 29, 1605071.

470 T. Kaji, T. Takenobu, A. F. Morpurgo and Y. Iwasa, Adv. Mater., 2009, 21, 3689-3693.

471 K. Tybrandt, K. C. Larsson, A. Richter-Dahlfors and M. Berggren, Proc. Natl. Acad. Sci. U. S. A., 2010, 107, 9929-9932.

472 H. Du, X. Lin, Z. Xu and D. Chu, J. Mater. Sci., 2015, 50, 5641-5673.

473 W. Chen, S. Horike, D. Umeyama, N. Ogiwara, T. Itakura, C. Tassel, Y. Goto, H. Kageyama and S. Kitagawa, Angew. Chem., Int. Ed., 2016, 55, 5195-5200.

474 N. Yanai, T. Uemura, S. Horike, S. Shimomura and S. Kitagawa, Chem. Commun., 2011, 47, 1722.

475 L. Gao, C.-Y. V. Li, K.-Y. Chan and Z.-N. Chen, J. Am. Chem. Soc., 2014, 136, 7209-7212.

476 T. Uemura, D. Hiramatsu, Y. Kubota, M. Takata and S. Kitagawa, Angew. Chem., 2007, 119, 5075-5078.

477 M. Inukai, S. Horike, T. Itakura, R. Shinozaki, N. Ogiwara, D. Umeyama, S. Nagarkar, Y. Nishiyama, M. Malon, A. Hayashi, T. Ohhara, R. Kiyanagi and S. Kitagawa, J. Am. Chem. Soc., 2016, 138, 8505-8511.

478 A. Karmakar, A. V. Desai and S. K. Ghosh, Coord. Chem. Rev., 2016, 307, 313-341.

479 J. F. Van Humbeck, M. L. Aubrey, A. Alsbaiee, R. Ameloot, G. W. Coates, W. R. Dichtel and J. R. Long, Chem. Sci., 2015, 6, 5499-5505.

480 M. L. Aubrey and J. R. Long, J. Am. Chem. Soc., 2015, 137, 13594-13602.

481 M. Dogru and T. Bein, Chem. Commun., 2014, 50, 5531-5546.

482 A. Yella, H. W. Lee, H. N. Tsao, C. Y. Yi, A. K. Chandiran, M. K. Nazeeruddin, E. W. G. Diau, C. Y. Yeh, S. M. Zakeeruddin and M. Gratzel, Science, 2011, 334, 629-634.

483 F. P. Doty, C. A. Bauer, A. J. Skulan, P. G. Grant and M. D. Allendorf, Adv. Mater., 2009, 21, 95-101.

484 G. Li, Z.-K. Tan, D. Di, M. L. Lai, L. Jiang, J. H.-W. Lim, R. H. Friend and N. C. Greenham, Nano Lett., 2015, 15, 2640-2644.

485 J. Yu, Y. Cui, H. Xu, Y. Yang, Z. Wang, B. Chen and G. Qian, Nat. Commun., 2013, 4, 2719.

486 H. He, E. Ma, Y. Cui, J. Yu, Y. Yang, T. Song, C.-D. Wu, X. Chen, B. Chen and G. Qian, Nat. Commun., 2016, 7, 11087.

487 I. Braun, G. Ihlein, F. Laeri, J. U. Nöckel, G. Schulz-Ekloff, F. Schüth, U. Vietze, Ö. Weiss and D. Wöhrle, Appl. Phys. B: Lasers Opt., 2000, 70, 335-343.

488 L. R. Mingabudinova, V. V. Vinogradov, V. A. Milichko, E. Hey-Hawkins and A. V. Vinogradov, Chem. Soc. Rev., 2016, 5408-5431.

489 S. Van Cleuvenbergen, I. Stassen, E. Gobechiya, Y. Zhang, K. Markey, D. E. De Vos, C. Kirschhock, B. Champagne, T. Verbiest and M. A. van der Veen, Chem. Mater., 2016, 28, 3203-3209.

490 M. T. Sebastian and H. Jantunen, Int. J. Appl. Ceram. Technol., 2010, 7, 415-434.

491 M. T. Sebastian, R. Ubic and H. Jantunen, Int. Mater. Rev., 2015, 60, 392-412.
492 Y. Sakata, S. Furukawa, M. Kondo, K. Hirai, N. Horike, Y. Takashima, H. Uehara, N. Louvain, M. Meilikhov, T. Tsuruoka, S. Isoda, W. Kosaka, O. Sakata and S. Kitagawa, Science, 2013, 339, 193-196.

493 B. H. Lee, B. Yoon, A. I. Abdulagatov, R. A. Hall and S. M. George, Adv. Funct. Mater., 2013, 23, 532-546.

494 S. B. Kumar and J. Guo, Nano Lett., 2012, 12, 1362-1366.

495 M. Ghorbani-Asl, S. Borini, A. Kuc and T. Heine, Phys. Rev.

B: Condens. Matter Mater. Phys., 2013, 87, 235434.

496 T. D. Bennett, J.-C. Tan, Y. Yue, E. Baxter, C. Ducati, N. J. Terrill, H. H.-M. Yeung, Z. Zhou, W. Chen, S. Henke, A. K. Cheetham and G. N. Greaves, Nat. Commun., 2015, 6, 8079.

497 D. Umeyama, S. Horike, M. Inukai, T. Itakura and S. Kitagawa, J. Am. Chem. Soc., 2015, 137, 864-870.

498 P. Raccuglia, K. C. Elbert, P. D. F. Adler, C. Falk, M. B. Wenny, A. Mollo, M. Zeller, S. A. Friedler, J. Schrier and A. J. Norquist, Nature, 2016, 533, 73-76.

499 A. Walsh, K. T. Butler and C. H. Hendon, MRS Bull., 2016, 41, 870-876.

500 P. Z. Moghadam, A. Li, S. B. Wiggin, A. Tao, A. G. P. Maloney, P. A. Wood, S. C. Ward and D. Fairen-Jimenez, Chem. Mater., 2017, 29, 2618-2625.

501 Y. G. Chung, J. Camp, M. Haranczyk, B. J. Sikora, W. Bury, V. Krungleviciute, T. Yildirim, O. K. Farha, D. S. Sholl and R. Q. Snurr, Chem. Mater., 2014, 26, 6185-6192.

502 D. Nazarian, J. S. Camp, Y. G. Chung, R. Q. Snurr and D. S. Sholl, Chem. Mater., 2016, 29, 2521-2528.

503 J. P. Dürholt, J. Keupp, R. Amabile and R. Schmid, www. mofplus.org, 2017.

504 E. L. First and C. A. Floudas, Microporous Mesoporous Mater., 2013, 165, 32-39.

505 M. O'Keeffe, M. A. Peskov, S. J. Ramsden and O. M. Yaghi, Acc. Chem. Res., 2008, 41, 1782-1789.

506 M. Pinheiro, R. L. Martin, C. H. Rycroft, A. Jones, E. Iglesia and M. Haranczyk, J. Mol. Graphics Modell., 2013, 44, 208-219.

507 K. B. Lausund and O. Nilsen, Nat. Commun., 2016, 7, 13578.

508 E. Ahvenniemi and M. Karppinen, Chem. Commun., 2016, 52, 1139-1142.

509 E. Ahvenniemi and M. Karppinen, Chem. Mater., 2016, 28, 6260-6265.

510 M. Nisula, J. Linnera, A. J. Karttunen and M. Karppinen, Chem. - Eur. J., 2017, 23, 2988-2992.

511 J.-O. Kim, K.-I. Min, H. Noh, D.-H. Kim, S.-Y. Park and D.P. Kim, Angew. Chem., Int. Ed., 2016, 55, 7116-7120.

512 C. Avci, J. Ariñez-Soriano, A. Carné-Sánchez, V. Guillerm, C. Carbonell, I. Imaz and D. Maspoch, Angew. Chem., Int. Ed., 2015, 54, 14417-14421.

513 D. Sheberla, J. C. Bachman, J. S. Elias, C.-J. Sun, Y. ShaoHorn and M. Dincă, Nat. Mater., 2016, 16, 220-224.

514 X.-Y. Cui, Z.-Y. Gu, D.-Q. Jiang, Y. Li, H.-F. Wang and X.-P. Yan, Anal. Chem., 2009, 81, 9771-9777.

515 C.-T. He, J.-Y. Tian, S.-Y. Liu, G. Ouyang, J.-P. Zhang and X.-M. Chen, Chem. Sci., 2013, 4, 351-356.

516 M. Peplow, Nature, 2015, 520, 148-150.

517 Nat. Chem., 2016, 8, 987. 